\title{
AN APPLICATION OF JOHN ELLIPSOIDS TO THE SZEGÖ KERNEL ON UNBOUNDED CONVEX DOMAINS
}

\author{
SOLEDAD BENGURIA
}

\begin{abstract}
We use convex geometry tools, in particular John ellipsoids, to obtain a size estimate for the Szegö kernel on the boundary of a class of unbounded convex domains in $\mathbb{C}^{n}$. Given a polynomial $b: \mathbb{R}^{n} \rightarrow \mathbb{R}$ satisfying a certain growth condition, we consider domains of the type $\Omega_{b}=\left\{z \in \mathbb{C}^{n+1}:\right.$ $\left.\operatorname{Im}\left[z_{n+1}\right]>b\left(\operatorname{Re}\left[z_{1}\right], \ldots, \operatorname{Re}\left[z_{n}\right]\right)\right\}$.
\end{abstract}

\section{INTRODUCTION}

The study of the behavior of Szegö kernels near the boundary of domains has been of great interest in the field of several complex variables during the past few decades. In this work we obtain a size estimate for the Szegö kernel on the boundary of a class of unbounded domains in $\mathbb{C}^{n}$. Our approach to this problem is the use of classical convex analysis techniques, and in particular an application of John ellipsoids.

1.1. Background. Given a strictly convex polynomial $b: \mathbb{R}^{n} \rightarrow \mathbb{R}$, consider the domain

$$
\Omega_{b}=\left\{\left(z_{1}, \ldots, z_{n+1}\right) \in \mathbb{C}^{n+1}: \operatorname{Im}\left[z_{n+1}\right]>b\left(\operatorname{Re}\left[z_{1}\right], \ldots, \operatorname{Re}\left[z_{n}\right]\right)\right\} .
$$

For unbounded domains of this type, it is convenient to define the Szegö projection as in [15]. We can identify the boundary $\partial \Omega_{b}$ with $\mathbb{C}^{n} \times \mathbb{R}$ so that a point $(\boldsymbol{z}, t) \in \mathbb{C}^{n} \times \mathbb{R}$ corresponds to $\left(\boldsymbol{z}, t+i b\left(\operatorname{Re}\left[z_{1}\right], \ldots, \operatorname{Re}\left[z_{n}\right]\right)\right) \in \partial \Omega_{b}$.

Let $\mathcal{O}\left(\Omega_{b}\right)$ be the set of holomorphic functions in $\Omega_{b}$. Given $F \in \mathcal{O}\left(\Omega_{b}\right)$ and $\epsilon>0$, set

$$
F_{\epsilon}(\boldsymbol{z}, t)=F\left(\boldsymbol{z}, t+i b\left(\operatorname{Re}\left[z_{1}\right], \ldots, \operatorname{Re}\left[z_{n}\right]\right)+i \epsilon\right) .
$$

The Hardy space $\mathcal{H}^{2}\left(\Omega_{b}\right)$ is defined as

$$
\mathcal{H}^{2}\left(\Omega_{b}\right)=\left\{F \in \mathcal{O}\left(\Omega_{b}\right): \sup _{\epsilon>0} \int_{\mathbb{C}^{n} \times \mathbb{R}}\left|F_{\epsilon}(\boldsymbol{z}, t)\right|^{2} d \boldsymbol{z} d t \equiv\|F\|_{\mathcal{H}^{2}}^{2}<\infty\right\} .
$$

Let $\rho\left(z_{1}, \ldots, z_{n+1}\right)=b\left(\operatorname{Re}\left[z_{1}\right], \ldots, \operatorname{Re}\left[z_{n}\right]\right)-\operatorname{Im}\left[z_{n+1}\right]$ be a defining function for the domain, i.e., $\Omega_{b}=\left\{\boldsymbol{z} \in \mathbb{C}^{n+1}: \rho(\boldsymbol{z})<0\right\}$ where $\rho \in C^{\infty}\left(\mathbb{C}^{n+1}\right)$ is such that $\nabla \rho \neq 0$ when $\rho=0$. A CauchyRiemann operator is an operator of the form $L=\sum_{j=1}^{n+1} a_{j} \frac{\partial}{\partial \overline{z_{j}}}$. We say that $L$ is tangential if in addition $L(\rho)=0$.

For a class of convex polynomials $b: \mathbb{R}^{n} \rightarrow \mathbb{R}$ satisfying a certain growth condition, we will define the Szegö projection $\Pi: L^{2}\left(\partial \Omega_{b}\right) \rightarrow \mathcal{H}^{2}\left(\Omega_{b}\right)$ to be the orthogonal projection from $L^{2}\left(\partial \Omega_{b}\right)$ to the closed subspace of functions $f \in L^{2}\left(\partial \Omega_{b}\right)$ that are annihilated in the sense of distributions by all tangential Cauchy-Riemann operators on $\partial \Omega_{b}$. For a thorough discussion on why such a map is

1991 Mathematics Subject Classification. 32A25 primary; 30C40 secondary.

Key words and phrases. Szegö kernel; John ellipsoids; unbounded convex domains. 
well-defined, refer to Appendix B on [28]. It can be shown that the Szegö projection is given by integration against a kernel. That is,

$$
\Pi[f](z)=\int_{\partial \Omega_{b}} S(z, w) f(w) d \sigma(w)
$$

where $d \sigma$ is an appropriate measure on $\partial \Omega_{b}$ (defined below, just before the statement of the Main Theorem). Here, $S(z, w)$, is called the Szegö kernel and is the object we study.

The growth condition that we will impose on the polynomials $b$ on this paper is the following.

Definition 1.1. Let $m_{1}, \ldots, m_{n}$ be positive integers. We will say that a polynomial $p: \mathbb{R}^{n} \rightarrow \mathbb{R}$ is of combined degree $\left(m_{1}, \ldots, m_{n}\right)$ if it is of the form

$$
p(\boldsymbol{x})=\sum_{\alpha} c_{\alpha} \boldsymbol{x}^{\alpha}
$$

where the exponents of its pure terms of highest order are $2 m_{1}, \ldots, 2 m_{n}$ respectively and each index $\alpha=\left(\alpha_{1}, \ldots, \alpha_{n}\right)$ satisfies

$$
\begin{aligned}
& \text { (1) } \frac{\alpha_{1}}{2 m_{1}}+\ldots+\frac{\alpha_{n}}{2 m_{n}} \leq 1 \\
& \text { (2) } \frac{\alpha_{1}}{2 m_{1}}+\ldots+\frac{\alpha_{n}}{2 m_{n}}=1 \text { if and only if there exists some } j \text { such that } \alpha_{j}=2 m_{j} \text {. }
\end{aligned}
$$

Example 1.2. The polynomial $p\left(x_{1}, x_{2}\right)=x_{1}^{2}+x_{1} x_{2}+x_{1}^{2} x_{2}^{2}+x_{1}^{4}+x_{2}^{6}$ is of combined degree $(2,3)$. However, the polynomial $\tilde{p}\left(x_{1}, x_{2}\right)=x_{1}^{2}+x_{1} x_{2}+x_{1}^{2} x_{2}^{3}+x_{1}^{4}+x_{2}^{6}$ is not a polynomial of combined degree.

Throughout the rest of this work we will assume that

$$
\Omega_{b}=\left\{\left(z_{1}, \ldots, z_{n+1}\right) \in \mathbb{C}^{n+1}: \operatorname{Im}\left[z_{n+1}\right]>b\left(\operatorname{Re}\left[z_{1}\right], \ldots, \operatorname{Re}\left[z_{n}\right]\right)\right\},
$$

where $b: \mathbb{R}^{n} \rightarrow \mathbb{R}$ is a strictly convex polynomial of combined degree $\left(m_{1}, \ldots, m_{n}\right)$. We will also identify $\partial \Omega_{b}$ with $\mathbb{R}^{n} \times \mathbb{R}^{n} \times \mathbb{R}$. That is, given $\left(\boldsymbol{z}, z_{n+1}\right) \in \mathbb{C}^{n+1}$, we write $\boldsymbol{z}=\boldsymbol{x}+i \boldsymbol{y}$, and denote a point on $\partial \Omega_{b}$ by using the notation $(\boldsymbol{x}, \boldsymbol{y}, t)$, where $t=\operatorname{Re}\left[z_{n+1}\right]$. As is [15], and to avoid degeneracy issues due to the unboundedness of the domain, we take Lebesgue measure $d \sigma=d \boldsymbol{x} d \boldsymbol{y} d t$ as the measure on the boundary.

We obtain the following size estimate for the Szegő kernel on the boundary of $\Omega_{b}$ :

Main Theorem. Let $(\boldsymbol{x}, \boldsymbol{y}, t)$ and $\left(\boldsymbol{x}^{\prime}, \boldsymbol{y}^{\prime}, t^{\prime}\right)$ be any two points in $\partial \Omega_{b}$. Define $\tilde{b}(\boldsymbol{v})=b\left(\boldsymbol{v}+\frac{\boldsymbol{x}+\boldsymbol{x}^{\prime}}{2}\right)-$ $\nabla b\left(\frac{\boldsymbol{x}+\boldsymbol{x}^{\prime}}{2}\right) \cdot \boldsymbol{v}-b\left(\frac{\boldsymbol{x}+\boldsymbol{x}^{\prime}}{2}\right) ; \delta\left(\boldsymbol{x}, \boldsymbol{x}^{\prime}\right)=b(\boldsymbol{x})+b\left(\boldsymbol{x}^{\prime}\right)-2 b\left(\frac{\boldsymbol{x}+\boldsymbol{x}^{\prime}}{2}\right) ;$ and $w=\left(t^{\prime}-t\right)+\nabla b\left(\frac{\boldsymbol{x}+\boldsymbol{x}^{\prime}}{2}\right) \cdot\left(\boldsymbol{y}^{\prime}-\boldsymbol{y}\right)$. Then

$$
\left|S\left((\boldsymbol{x}, \boldsymbol{y}, t) ;\left(\boldsymbol{x}^{\prime}, \boldsymbol{y}^{\prime}, t^{\prime}\right)\right)\right| \leq \frac{C}{\sqrt{\delta^{2}+\widetilde{b}\left(\boldsymbol{y}-\boldsymbol{y}^{\prime}\right)^{2}+w^{2}}\left|\left\{\boldsymbol{v}: \tilde{b}(\boldsymbol{v})<\sqrt{\delta^{2}+\widetilde{b}\left(\boldsymbol{y}-\boldsymbol{y}^{\prime}\right)^{2}+w^{2}}\right\}\right|^{2}} .
$$

Here the constant $C$ depends on the exponents $\left\{m_{1}, \ldots, m_{n}\right\}$ and the dimension of the space, but is otherwise independent of the choice of $b$ and of the two given points. 
Remark 1.3. The condition that $b$ is of combined degree is sufficient to ensure the existence of estimates as above. However, similar results could probably be obtained under weaker assumptions (e.g., a finite-type assumption). The methods we use in this work, though, rely heavily on the combined degree hypothesis. New methods would have to be developed to generalize this result. In particular, the proof of Claims 4.3 and 4.5 would need to be adapted. The result of these claims (especially of the first one) are used repeatedly throughout the paper. The fact that $b$ is a polynomial function also plays an important role throughout this work (see, e.g., Claims 4.8, 4.11, and 5.4).

For bounded domains $\Omega$ the Szegö kernel has been extensively studied. Among others, the works by Gindikin [13], Fefferman [8], Boutet de Monvel and Sjöstrand [4], Phong and Stein [29], Boas [2, 3], Christ [7], Fefferman, Kohn, Machedon [9], Machedon [22], McNeal and Stein [24], Lanzani and Stein [20] [21], and Krantz [18] [19], have given great insight on the behavior of the Szegö kernel and the Szegö projection. Much less is known about the Szegö kernel on unbounded domains $\Omega$. Rather than broad general results, only particular domains have been studied in the latter case.

When studying the Szegö kernels for unbounded domains, domains of the type $\Omega=\left\{\left(\boldsymbol{z}, z_{n+1}\right) \in\right.$ $\left.\mathbb{C}^{n} \times \mathbb{C}: \operatorname{Im}\left[z_{n+1}\right]>\phi(\boldsymbol{z})\right\}$ for different choices of functions $\phi$ have been of particular interest. In fact, when $n=1$ the Szegö projection and kernel on such domains have been extensively studied. For example, Greiner and Stein [14] obtain a closed formula for the Szegö kernel for $\phi(z)=|z|^{2 k}, k \in \mathbb{N}$. The singularities of the Szegő kernel have been studied by Haslinger [16] when $\phi(z)=|\operatorname{Re}[z]|^{\alpha}$, $\alpha>\frac{4}{3}$; by Carracino [6] for a particular choice of a non-convex $\phi$; by Gilliam and Halfpap [11], [12] when $\phi$ is a non-convex even degree polynomial with positive leading coefficient; and by Halfpap, Nagel and Wainger [15], who consider, among others, functions such that $\phi(z)=\exp \left(-|z|^{-a}\right), a>0$, for $|z|$ small and $\phi(z)=z^{2 m}$ for $|z|$ large. Nagel [25] studies the Szegö kernel on the boundary of domains of the kind $\Omega=\left\{z \in \mathbb{C}^{2}: \operatorname{Im}\left[z_{2}\right]>\phi\left(\operatorname{Re}\left[z_{1}\right]\right)\right\}$, where $\phi$ is a subharmonic, non-harmonic polynomial with the property that $\Delta \phi(z)=\Delta \phi(x+i y)$ is independent of $y$. He shows that in this case the Szegö kernel is bounded by $|B|^{-1}$, where $|B|$ is the volume of a certain non-isotropic ball. Nagel's result was later generalized by Nagel, Rosay, Stein and Wainger [26] [27] to domains where $\phi$ is a subharmonic, non-harmonic polynomial in $\mathbb{C}$. Furthermore, they obtain similar estimates for the derivatives of the Szegő kernel, and use these to obtain $L^{p}$ bounds for the Szegö projection. See also [22] for a related problem. More recently, Peterson [28] considered domains where $\phi$ is a smooth, subharmonic, nonharmonic function and $\partial \Omega$ satisfies a uniform finite-type hypothesis of order $m$.

Less progress has been made in the case $n>1$. See, however, [10], and the references therein. In [10], Franciscs and Hanges take $\phi(\boldsymbol{z}, \boldsymbol{\xi})=\|\boldsymbol{z}\|^{2}+\|\xi\|^{2 p}$ for $\boldsymbol{z} \in \mathbb{C}^{n}, \xi \in \mathbb{C}^{m}, n \geq 0, m \geq 1$ and $p$ a positive integer, and obtain a closed formula for the Szegö kernel.

It was the goal of obtaining similar results to those of Nagel [25], but for $n>1$, that led me to work on the problem at hand. One of the main difficulties of the problem in several dimensions stems from the fact that the polynomial $b$ we consider can exhibit different growth rates along different directions. This is where the John ellipsoids come into play, allowing one to introduce a rescaling that takes care of this issue.

In [30], Raich and Tinker study a similar problem. They consider domains $\Omega=\left\{(z, \boldsymbol{w}) \in \mathbb{C} \times \mathbb{C}^{n}\right.$ : $\operatorname{Im}[\boldsymbol{w}]=P(\operatorname{Re}[z])\}$, where $P=\left(a_{1} p, \ldots, a_{n} p\right)$ with $p: \mathbb{R} \rightarrow \mathbb{R}$ a convex polynomial, $a_{n}=1$, and $\boldsymbol{a}=\left(a_{1}, \ldots, a_{n}\right) \in \mathbb{R}^{n}$. They obtain a bound for the Szegö kernel and its derivatives in terms of the 
volume of a ball defined by a certain pseudometric, as well as an explicit formula for the Szegö kernel when $p(x)=x^{2}$.

1.2. Methods. Except for the setup of the problem, which is outlined in Section 2, all the methods we use are classical convex analysis techniques. In fact, an application of John ellipsoids is the key ingredient in the proof of the Main Theorem.

In Section 2 we derive an integral formula for the Szegö kernel. Our estimates all follow from a study of this integral expression, given by

$$
S\left((\boldsymbol{x}, \boldsymbol{y}, t) ;\left(\boldsymbol{x}^{\prime}, \boldsymbol{y}^{\prime}, t^{\prime}\right)\right)=\int_{0}^{\infty} e^{-2 \pi \tau\left[b\left(\boldsymbol{x}^{\prime}\right)+b(\boldsymbol{x})+i\left(t^{\prime}-t\right)\right]}\left(\int_{\mathbb{R}^{n}} \frac{e^{2 \pi \boldsymbol{\eta} \cdot\left[\boldsymbol{x}+\boldsymbol{x}^{\prime}-i\left(\boldsymbol{y}^{\prime}-\boldsymbol{y}\right)\right]}}{\int_{\mathbb{R}^{n}} e^{4 \pi[\boldsymbol{\eta} \cdot \boldsymbol{v}-b(\boldsymbol{v}) \tau]} d \boldsymbol{v}} d \boldsymbol{\eta}\right) d \tau .
$$

In Sections 3 and 4 we build the tools that we use in the proof of the Main Theorem, which is presented in Section 5. We devote Section 3 to a study of the coefficients of convex polynomials in several variables. In the one-variable case it was shown in [5] that the absolute value of the coefficients of a convex polynomial with no constant or linear terms can be bounded, up to a constant that depends only on the degree of the polynomial, by the value of the polynomial at 1 . It is not possible to obtain such a bound in more variables, since the polynomial might be growing in some directions but not along others. However, we show that the absolute value of the coefficients can be bounded by the average of the polynomial over a circle of arbitrary positive radius, up to a constant that only depends on the degree of the polynomial and the chosen radius. In Section 4 we use this result to prove a technical lemma that will be key in dealing with the denominator integral of equation (3).

In Section 5 we present the proof of the Main Theorem. The proof is, at its core, an application of John ellipsoids. We introduce a change of variables in the integral expression for the Szegö kernel comprised of factors defined by the length of the axes of the unique maximal inscribed ellipsoid associated to a symmetrization of the convex body $R=\{\boldsymbol{v}: \tau \tilde{b}(\boldsymbol{v}) \leq 1\}$. The construction of these factors is presented in Section 5.1, and it is from the product of these factors (which appear in the denominator as the Jacobian of the change of variables), that the volume expression in the estimate given in equation (2) is obtained.

So as to make the computations simpler, we have split the proof of the Main Theorem into the proof of three separate bounds. The first bound, in terms of $\delta$, is given in Section 5.2.1; the second bound, in terms of $\widetilde{b}\left(\boldsymbol{y}-\boldsymbol{y}^{\prime}\right)$, is presented in Section 5.2.2; and the bound in terms of $w$ is given in Section 5.2.3. These bounds are then combined to yield the estimate of the Main Theorem in Section 5.2 .4 .

Geometric tools, such as the ones we employ in this paper, have often been used in the study of the Szegő kernel. For example, similar geometric ideas are used by McNeal and Stein [24] to obtain a bound for the Szegö kernel $S(z, w)$ for smoothly bounded convex domains $\Omega$ of finite type in $\mathbb{C}^{n}$ in terms of the smallest tent in $\partial \Omega$ containing $z$ and $w$ (see also [23]). That is, they show that for smoothly bounded convex domains of finite type in $\mathbb{C}^{n}$, there exists a constant $C$ so that for all $z, w \in \bar{\Omega} \times \bar{\Omega} \backslash\{$ diagonal in $\partial \Omega\}$, 


$$
|S(z, w)| \leq \frac{C}{|T(z, \gamma)|}
$$

Here $T(z, \gamma)=P_{\gamma}(\pi(z)) \cap \bar{\Omega}$; the projection $\pi: U \rightarrow \partial \Omega$ is a smooth map such that if $b \in \partial \Omega, \pi(b)=b$ and $\pi^{-1}(b)$ is a smooth curve, transversally intersecting $\partial \Omega$ at $b$; and $\gamma=|r(z)|+|r(w)|+\inf \{\epsilon>0$ : $w \in T(z, \epsilon)\}$. The geometric constructions used in [23] and [24] are based on the length of the sides of a certain polydisc, as opposed to the lengths of the axes of an ellipsoid. The use of John ellipsoids, however, seems more natural for the domains we consider. In fact, one of the key components of our proof is the use of universal bounds for the coefficient of convex polynomials in terms of the average of the polynomials over circles of arbitrary positive radius (as described above, and in more detail in Section 3). Thus, it makes sense to consider ellipsoids (which can be rescaled into spheres), rather than polydiscs. The particular tools we employ (i.e, the approximation by John ellipsoids) have not been used before in this context and provide a new approach to the problem.

\section{Preliminaries}

In this section we derive an integral formula for the Szegő kernel for the domains under consideration. We follow the analogous derivation for the one-dimensional case found in [25].

Proposition 2.1. The Szegö kernel on the boundary of domains of the type $\Omega_{b}=\left\{\boldsymbol{z} \in \mathbb{C}^{n+1}\right.$ : $\left.\operatorname{Im}\left[z_{n+1}\right]>b\left(\operatorname{Re}\left[z_{1}\right], \ldots, \operatorname{Re}\left[z_{n}\right]\right)\right\}$ where $b: \mathbb{R}^{n} \rightarrow \mathbb{R}$ is a convex polynomial of combined degree is given by

$$
S\left((\boldsymbol{x}, \boldsymbol{y}, t) ;\left(\boldsymbol{x}^{\prime}, \boldsymbol{y}^{\prime}, t^{\prime}\right)\right)=\int_{0}^{\infty} e^{-2 \pi \tau\left[b\left(\boldsymbol{x}^{\prime}\right)+b(\boldsymbol{x})+i\left(t^{\prime}-t\right)\right]}\left(\int_{\mathbb{R}^{n}} \frac{e^{2 \pi \boldsymbol{\eta} \cdot\left[\boldsymbol{x}+\boldsymbol{x}^{\prime}-i\left(\boldsymbol{y}^{\prime}-\boldsymbol{y}\right)\right]}}{\int_{\mathbb{R}^{n}} e^{4 \pi[\boldsymbol{\eta} \cdot \boldsymbol{v}-b(\boldsymbol{v}) \tau]} d \boldsymbol{v}} d \boldsymbol{\eta}\right) d \tau,
$$

where $(\boldsymbol{x}, \boldsymbol{y}, t)$ and $\left(\boldsymbol{x}^{\prime}, \boldsymbol{y}^{\prime}, t^{\prime}\right)$ are any two points on $\partial \Omega_{b}$.

Proof. Let

$$
\begin{aligned}
\rho\left(z_{1}, \ldots, z_{n+1}\right) & =b\left(\operatorname{Re}\left[z_{1}\right], \ldots, \operatorname{Re}\left[z_{n}\right]\right)-\operatorname{Im}\left[z_{n+1}\right] \\
& =b\left(\frac{z_{1}+\bar{z}_{1}}{2}, \ldots, \frac{z_{n}+\bar{z}_{n}}{2}\right)-\frac{z_{n+1}-\bar{z}_{n+1}}{2 i}
\end{aligned}
$$

be a defining function for our domain. The Szegő projection is the orthogonal projection $\Pi$ : $L^{2}\left(\partial \Omega_{b}\right) \rightarrow H^{2}\left(\partial \Omega_{b}\right)$. It can be shown (see, e.g., [15], [1]), that $H^{2}\left(\partial \Omega_{b}\right)$ as defined in equation (1) is equivalent to the space

$$
\left\{f \in L^{2}\left(\partial \Omega_{b}\right): \bar{Z}(f)=0 \text { as a distribution, for all } \bar{Z} \in T^{0,1}\left(\partial \Omega_{b}\right)\right\} .
$$

We begin by finding a base for the tangential Cauchy-Riemann operators. We can let

$$
\bar{Z}_{j}=2\left(\frac{\partial}{\partial \bar{z}_{j}}+A_{j}\left(z_{1}, \ldots, z_{n}\right) \frac{\partial}{\partial \bar{z}_{n+1}}\right) \quad j=1, \ldots, n .
$$

For these operators to be tangential they must satisfy $\bar{Z}_{j}(\rho)=0$. Thus, 


$$
\bar{Z}_{j}=2\left(\frac{\partial}{\partial \bar{z}_{j}}-i \frac{\partial b}{\partial x_{j}}(\boldsymbol{x}) \frac{\partial}{\partial \bar{z}_{n+1}}\right) \quad j=1, \ldots, n
$$

are a basis for the space of tangential Cauchy-Riemann operators for our domain in $\mathbb{C}^{n+1}$. We can identify $\partial \Omega_{b}$ with $\mathbb{C}^{n} \times \mathbb{R}$ via the diffeomorphism

$$
\left(z_{1}, \ldots, z_{n}, t\right) \in \mathbb{C}^{n} \times \mathbb{R} \leftrightarrow\left(z_{1}, \ldots, z_{n}, t+i b\left(\operatorname{Re}\left[z_{1}\right], \ldots, \operatorname{Re}\left[z_{n}\right]\right)\right) \in \partial \Omega_{b}
$$

Our operators $\bar{Z}_{j}$ are operators in $\mathbb{C}^{n+1}$. The pushforward of these operators to $\mathbb{C}^{n} \times \mathbb{R}$ is

$$
\bar{Z}_{j}=\frac{\partial}{\partial x_{j}}+i\left(\frac{\partial}{\partial y_{j}}-\frac{\partial b}{\partial x_{j}}(\boldsymbol{x}) \frac{\partial}{\partial t}\right) .
$$

Lemma 2.2. Given $\boldsymbol{x} \in \mathbb{R}^{n}, \boldsymbol{\eta} \in \mathbb{R}^{n}$, and $\tau \in \mathbb{R}$, let

$$
\mathcal{M}[g](\boldsymbol{x}, \boldsymbol{\eta}, \tau)=e^{-2 \pi[\boldsymbol{\eta} \cdot \boldsymbol{x}-b(\boldsymbol{x}) \tau]} g(\boldsymbol{x}, \boldsymbol{\eta}, \tau),
$$

and define the partial Fourier transform

$$
\mathcal{F}[f](\boldsymbol{x}, \boldsymbol{y}, \tau)=\hat{f}(\boldsymbol{x}, \boldsymbol{\eta}, \tau)=\int_{\mathbb{R}^{n+1}} e^{-2 \pi i(\boldsymbol{y} \cdot \boldsymbol{\eta}+t \tau)} f(\boldsymbol{x}, \boldsymbol{y}, t) d \boldsymbol{y} d t
$$

Then

$$
\mathcal{M}: L^{2}\left(\mathbb{R}^{2 n+1}, d \boldsymbol{x} d \boldsymbol{\eta} d \tau\right) \rightarrow L^{2}\left(\mathbb{R}^{2 n+1}, e^{4 \pi[\boldsymbol{\eta} \cdot \boldsymbol{x}-b(\boldsymbol{x}) \tau]} d \boldsymbol{x} d \boldsymbol{\eta} d \tau\right)
$$

is an isometry for $f \in L^{2}\left(\mathbb{R}^{2 n+1}, d \boldsymbol{x} d \boldsymbol{\eta} d \tau\right)$, and

$$
\bar{Z}_{j}[f]=\mathcal{F}^{-1} \mathcal{M}^{-1} \frac{\partial}{\partial x_{j}} \mathcal{M F}[f] \quad j=1, \ldots n .
$$

Proof. It is easy to check that $\mathcal{M}$ is an isometry in this weighted $L^{2}$ space. Also,

$$
\begin{aligned}
\bar{Z}_{j}[f] & =\bar{Z}_{j} \mathcal{F}^{-1}(\hat{f}) \\
& =\int_{\mathbb{R}^{n+1}} e^{2 \pi i(\boldsymbol{y} \cdot \boldsymbol{\eta}+t \tau)}\left(\frac{\partial \hat{f}(\boldsymbol{x}, \boldsymbol{\eta}, \tau)}{\partial x_{j}}-2 \pi \eta_{j} \hat{f}(\boldsymbol{x}, \boldsymbol{\eta}, \tau)+\frac{\partial b}{\partial x_{j}}(\boldsymbol{x}) 2 \pi \tau \hat{f}(\boldsymbol{x}, \boldsymbol{\eta}, \tau)\right) d \boldsymbol{\eta} d \tau \\
& =\int_{\mathbb{R}^{n+1}} e^{2 \pi i(\boldsymbol{y} \cdot \boldsymbol{\eta}+t \tau)} e^{2 \pi[\boldsymbol{\eta} \boldsymbol{x}-b(\boldsymbol{x}) \tau]} \frac{\partial}{\partial x_{j}}\left(e^{-2 \pi[\boldsymbol{\eta} \cdot \boldsymbol{x}-b(\boldsymbol{x}) \tau]} \hat{f}(\boldsymbol{x}, \boldsymbol{\eta}, \tau)\right) d \boldsymbol{\eta} d \tau \\
& =\mathcal{F}^{-1} \mathcal{M}^{-1} \frac{\partial}{\partial x_{j}} \mathcal{M} \mathcal{F}[f] .
\end{aligned}
$$

Since $\mathcal{F}$ and $\mathcal{M}$ are isometries, instead of projecting onto the null space of the tangential CauchyRiemann operators we can project onto the closed subspace of functions in $L^{2}\left(\mathbb{R}^{2 n+1}, e^{4 \pi[\boldsymbol{\eta} \cdot \boldsymbol{x}-b(\boldsymbol{x}) \tau]}\right.$ $d \boldsymbol{x} d \boldsymbol{\eta} d \tau)$ which are a.e. constant in $x$.

More precisely, as in [25], let 


$$
\Sigma=\left\{(\boldsymbol{\eta}, \tau) \in \mathbb{R}^{n+1} \mid \int_{\mathbb{R}^{n}} e^{4 \pi[\boldsymbol{\eta} \cdot \boldsymbol{x}-b(\boldsymbol{x}) \tau]} d \boldsymbol{x}<\infty\right\}
$$

Then, because of the growth hypothesis on $b$,

$$
\Sigma=\left\{(\boldsymbol{\eta}, \tau) \in \mathbb{R}^{n+1} \mid \tau>0\right\} .
$$

This follows from the fact that $b$ is positive and grows at least quadratically in all directions.

Let $\widehat{\Pi}_{\boldsymbol{\eta}, \tau}$ be the projection of $L^{2}\left(\mathbb{R}^{n}, e^{4 \pi[\boldsymbol{\eta} \cdot \boldsymbol{x}-b(\boldsymbol{x}) \tau]} d \boldsymbol{x}\right)$ onto the constants if $(\boldsymbol{\eta}, \tau) \in \Sigma$, and let $\widehat{\Pi}_{\boldsymbol{\eta}, \tau}=0$ otherwise. Then, if $(\boldsymbol{\eta}, \tau) \in \Sigma$,

$$
\widehat{\Pi}_{\boldsymbol{\eta}, \tau} g=\frac{<g, 1>1}{<1,1>}=\int_{\mathbb{R}^{n}} g\left(\boldsymbol{x}^{\prime}\right)\left(\frac{e^{4 \pi\left[\boldsymbol{\eta} \cdot \boldsymbol{x}^{\prime}-b\left(\boldsymbol{x}^{\prime}\right) \tau\right]}}{\int_{\mathbb{R}^{n}} e^{4 \pi[\boldsymbol{\eta} \cdot \boldsymbol{v}-b(\boldsymbol{v}) \tau]} d \boldsymbol{v}}\right) d \boldsymbol{x}^{\prime} .
$$

We define the projection

$$
\widehat{\Pi}: L^{2}\left(\mathbb{R}^{2 n+1}, e^{4 \pi[\boldsymbol{\eta} \cdot \boldsymbol{x}-b(\boldsymbol{x}) \tau]} d \boldsymbol{x} d \boldsymbol{\eta} d \tau\right) \rightarrow L^{2}\left(\mathbb{R}^{2 n+1}, e^{4 \pi[\boldsymbol{\eta} \cdot \boldsymbol{x}-b(\boldsymbol{x}) \tau]} d \boldsymbol{x} d \boldsymbol{\eta} d \tau\right)
$$

by

$$
\widehat{\Pi} g(\boldsymbol{x}, \boldsymbol{\eta}, \tau)=\widehat{\Pi}_{\boldsymbol{\eta}, \tau}\left(g_{\boldsymbol{\eta}, \tau}\right)(\boldsymbol{x}),
$$

where $g_{\boldsymbol{\eta}, \tau}(\boldsymbol{x})=g(\boldsymbol{x}, \boldsymbol{\eta}, \tau)$. Then $\Pi[f]=\mathcal{F}^{-1} \mathcal{M}^{-1} \widehat{\Pi} \mathcal{M} \mathcal{F}[f]$ is the projection from $L^{2}\left(\mathbb{R}^{2 n+1}, d \boldsymbol{x} d \boldsymbol{y} d t\right)$ onto the null space of the tangential Cauchy-Riemann operators. Thus, if $f \in L^{2}\left(\mathbb{R}^{2 n+1}, d \boldsymbol{x} d \boldsymbol{y} d t\right)$, the Szegö projection is given by

$$
\Pi[f](\boldsymbol{x}, \boldsymbol{y}, t)=\mathcal{F}^{-1} \mathcal{M}^{-1} \widehat{\Pi} \mathcal{M} \mathcal{F}[f](\boldsymbol{x}, \boldsymbol{y}, t)=\int_{\mathbb{R}^{2 n+1}} f\left(\boldsymbol{x}^{\prime}, \boldsymbol{y}^{\prime}, t^{\prime}\right) S\left((\boldsymbol{x}, \boldsymbol{y}, t) ;\left(\boldsymbol{x}^{\prime}, \boldsymbol{y}^{\prime}, t^{\prime}\right)\right) d \boldsymbol{x}^{\prime} d \boldsymbol{y}^{\prime} d t^{\prime}
$$

where the Szegő kernel is given by

$$
S\left((\boldsymbol{x}, \boldsymbol{y}, t) ;\left(\boldsymbol{x}^{\prime}, \boldsymbol{y}^{\prime}, t^{\prime}\right)\right)=\int_{0}^{\infty} e^{-2 \pi \tau\left[b\left(\boldsymbol{x}^{\prime}\right)+b(\boldsymbol{x})+i\left(t^{\prime}-t\right)\right]}\left(\int_{\mathbb{R}^{n}} \frac{e^{2 \pi \boldsymbol{\eta} \cdot\left[\boldsymbol{x}+\boldsymbol{x}^{\prime}-i\left(y^{\prime}-y\right)\right]}}{\int_{\mathbb{R}^{n}} e^{4 \pi[\boldsymbol{\eta} \cdot \boldsymbol{v}-b(\boldsymbol{v}) \tau]} d \boldsymbol{v}} d \boldsymbol{\eta}\right) d \tau .
$$

This finishes the proof of Proposition 2.1. 


\section{Coefficients of CONVEX POlynomials of SEVERAl VARIABles}

In this section we obtain bounds for the absolute value of the coefficients of convex polynomials of several variables with no constant or linear terms.

Theorem 3.1. Let $\Gamma(M)=\left\{\left(\alpha_{1}, \ldots, \alpha_{n}\right) \in \mathbb{N}^{n}: 2 \leq|\alpha| \leq M\right\}$. Let $S(M)$ be the set of convex polynomials of the form $g(\boldsymbol{v})=\sum_{\alpha \in \Gamma(M)} c_{\alpha} \boldsymbol{v}^{\alpha}$. Then for any fixed $a>0$, there exists a positive constant $C(M, a)$ that depends only on $M$ and the constant a such that if $g \in S(M)$,

$$
\sum_{\alpha \in \Gamma(M)}\left|c_{\alpha}\right| \leq C(M, a) \int_{|\sigma|=a} g(\sigma) d \sigma .
$$

Remark 3.2. This is a generalization of the result in one variable by Bruna, Nagel and Wainger in [5] (Lemma 2.1).

Proof. Let $a>0$ be a fixed positive constant and let $|\Gamma(M)|$ denote the cardinality of the set of indices $\Gamma(M)$. We identify the space $S(M)$ with a cone in $\mathbb{R}^{|\Gamma(M)|}$ via the identification

$$
g(\boldsymbol{v})=\sum_{\alpha \in \Gamma(M)} c_{\alpha} \boldsymbol{v}^{\alpha} \in S(M) \leftrightarrow\left(c_{1}, \ldots, c_{|\Gamma(M)|}\right) \in \mathbb{R}^{|\Gamma(M)|},
$$

where $c_{j}$ corresponds to the coefficient $c_{\alpha}$ for the $j^{\text {th }}$ element $\alpha$ in $\Gamma(M)$, under some fixed but arbitrary ordering of $\Gamma(M)$.

Let

$$
\Sigma_{M}=\left\{g(\boldsymbol{v})=\sum_{\alpha \in \Gamma(M)} c_{\alpha} \boldsymbol{v}^{\alpha} \in S(M): \sum_{\alpha \in \Gamma(M)}\left|c_{\alpha}\right|=1\right\}
$$

We claim that $\Sigma_{M}$ is a compact subset of the cone $S(M)$. In fact, let $\left\{\boldsymbol{c}_{\boldsymbol{n}}\right\}_{n \in \mathbb{N}}$ in $\mathbb{R}^{|\Gamma(M)|}$ be a sequence of tuples associated to a sequence of polynomials $\left\{q_{n}\right\}_{n \in \mathbb{N}}$ in $\Sigma_{M}$. Since $\left\{\boldsymbol{c}_{n}\right\}_{n \in \mathbb{N}}$ is a sequence contained in the compact set $B_{M}=\left\{\left(c_{1}, \ldots, c_{|\Gamma(M)|}\right) \in \mathbb{R}^{|\Gamma(m)|}: \sum_{1 \leq j \leq|\Gamma(M)|}\left|c_{j}\right|=1\right\}$, it has a convergent subsequence $\left\{\boldsymbol{c}_{\boldsymbol{n}_{\boldsymbol{i}}}\right\}_{n_{i} \in \mathbb{N}}$. Let $\boldsymbol{c}$ be the limit of this subsequence, and let $q$ be the polynomial associated to this tuple. We claim that $q$ is an element of $\Sigma_{M}$. In fact, the identification preserves the degree of the polynomial and the fact that there are no constant or linear terms. Also, since $\boldsymbol{c}$ is an element of $B_{M}$, it satisfies that $\sum_{1 \leq j \leq|\Gamma(M)|}\left|c_{j}\right|=1$. Thus, is suffices to show that $q$ is convex. This follows easily, since given any polynomial $q_{n_{i}}$ associated to an element of the convergent subsequence $\left\{\boldsymbol{c}_{\boldsymbol{n}_{\boldsymbol{i}}}\right\}_{n_{i} \in \mathbb{N}}$, we have that $q_{n_{i}}(\alpha \boldsymbol{x}+(1-\alpha) \boldsymbol{y}) \leq \alpha q_{n_{i}}(\boldsymbol{x})+(1-\alpha) q_{n_{i}}(\boldsymbol{y})$ for all $0 \leq \alpha \leq 1$ and for all points $\boldsymbol{x}, \boldsymbol{y}$ in $\mathbb{R}^{n}$. Thus, and since $q_{n_{i}}(\alpha \boldsymbol{x}+(1-\alpha) \boldsymbol{y}) \rightarrow q(\alpha \boldsymbol{x}+(1-\alpha) \boldsymbol{y})$; $\alpha q_{n_{1}}(\boldsymbol{x}) \rightarrow \alpha q(\boldsymbol{x})$; and $(1-\alpha) q_{n_{i}}(\boldsymbol{y}) \rightarrow(1-\alpha) q(\boldsymbol{y})$, the convexity of $q$ follows immediately.

Let

$$
\Phi_{I}(g)=\frac{1}{\omega_{n}(a)} \int_{|\sigma|=a} g(\sigma) d \sigma
$$

where $\omega_{n}(a)$ is the surface area of the sphere of radius $a$ in $\mathbb{R}^{n}$ and

$$
\Phi_{I I}(g)=\sum_{\alpha \in \Gamma(M)}\left|c_{\alpha}\right|
$$

Notice that these functions are continuous on $S(M)$, and that $\Phi_{I I}(g)=1$ on $\Sigma_{M}$. 
We claim that $\Phi_{I}(g)$ is strictly positive on $\Sigma_{M}$. In fact, since $g$ is convex, $g(\mathbf{0})=0$ and $\nabla g(\mathbf{0})=\mathbf{0}$ it follows that $g$ is nonnegative. Moreover, on $\Sigma_{M}$ at least one of the coefficients of $g$ must be different from zero, so $g$ can not be the zero polynomial. Thus $g$ must be positive almost everywhere. In particular, the average over the circle of radius $a$ must be strictly positive.

Therefore, and since $\Phi_{I}(g)$ is continuous as a function of $g$, it attains a minimum in $\Sigma_{M}$, and this minimum is strictly positive. Thus, and since $\Phi_{I I}(g)=1$ on $\Sigma_{M}$, there exists a constant $C>0$ such that for any $g \in \Sigma_{M}$,

$$
\Phi_{I}(g) \geq C=C \Phi_{I I}(g) .
$$

That is,

$$
\frac{1}{\omega_{n}(a)} \int_{|\sigma|=a} g(\sigma) d \sigma \geq C \Phi_{I I}(g)=C \sum_{\alpha \in \Gamma(M)}\left|c_{\alpha}\right|,
$$

as desired.

Consider now a polynomial $g(\boldsymbol{v})=\sum_{\alpha \in \Gamma(M)} c_{\alpha} \boldsymbol{v}^{\alpha} \in S(M)$, but which is not necessarily in $\Sigma_{M}$. Then let $h(\boldsymbol{v})=\sum_{\alpha \in \Gamma(M)} b_{\alpha} \boldsymbol{v}^{\alpha}$ where

$$
b_{\alpha}=\frac{c_{\alpha}}{\sum_{\beta \in \Gamma(M)}\left|c_{\beta}\right|}
$$

so that $\sum_{\alpha \in \Gamma(M)}\left|b_{\alpha}\right|=1$ and $h(\boldsymbol{v}) \in \Sigma_{M}$. It follows from the previous case that

That is,

$$
\frac{1}{\omega_{n}(a)} \int_{|\sigma|=a} h(\sigma) d \sigma \geq C
$$

This gives the desired inequality.

$$
\frac{1}{\omega_{n}(a)} \int_{|\sigma|=a} \frac{g(\sigma)}{\sum_{\beta \in \Gamma}\left|c_{\beta}\right|} d \sigma \geq C
$$

Corollary 3.3. Let $g(\boldsymbol{v})=\sum_{\alpha} c_{\alpha} \boldsymbol{v}^{\alpha}$ be a convex polynomial such that $g(\mathbf{0})=0$ and $\nabla g(\mathbf{0})=\mathbf{0}$. Suppose there exist two positive constants $A$ and $B$ such that

$$
\{\boldsymbol{v}:|\boldsymbol{v}| \leq A\} \subseteq\{\boldsymbol{v}: g(\boldsymbol{v}) \leq 1\} \subseteq\{\boldsymbol{v}:|\boldsymbol{v}| \leq B\} .
$$

Then there exists a constant $C$ that depends only on $A$ and the degree of $g$ such that

$$
\sum_{\alpha}\left|c_{\alpha}\right| \leq C
$$

Moreover, for any point $\boldsymbol{x}=\left(x_{1}, \ldots, x_{n}\right)$ on the sphere of radius $A$, there exist constants $C_{1}>0$, $C_{2}>0$ that depend only on $A, B$ and the degree of $g$ such that

$$
g(\boldsymbol{x}) \geq C_{1} \geq C_{2} \sum_{\alpha}\left|c_{\alpha}\right|
$$

Remark 3.4. The bound given by equation (10) can be obtained using just the left containment, i.e, the existence of a constant $A>0$ such that $\{\boldsymbol{v}:|\boldsymbol{v}| \leq A\} \subseteq\{\boldsymbol{v}: g(\boldsymbol{v}) \leq 1\}$. The second bound, however, requires the existence of both an inner and an outer ball. 
Proof. The first result follows immediately from the previous claim. In fact, we showed that

$$
\sum_{\alpha}\left|c_{\alpha}\right| \leq C \int_{|\sigma|=A} g(\sigma) d \sigma
$$

But by (9) we have that $g(\sigma) \leq 1$ for all $\sigma$ such that $|\sigma|=A$. The result follows.

Observe that the bound $g(\boldsymbol{x}) \geq C_{2} \sum_{\alpha}\left|c_{\alpha}\right|$ will be an immediate consequence of the above bound on the coefficients once we show that $g(\boldsymbol{x}) \geq C_{1}$.

The proof of equation (11) requires the use of Lemma 2.1 of [5]. The lemma states that given a convex polynomial of one variable of degree $M$ of the form

$$
p(t)=\sum_{j=2}^{M} a_{j} t^{j}
$$

there exists a constant $C_{M}>0$ that depends only on $M$ such that

$$
C_{M} \sum_{j=2}^{M}\left|a_{j}\right| t^{j} \leq p(t) \leq \sum_{j=2}^{M}\left|a_{j}\right| t^{j} \quad \forall t \geq 0 .
$$

In particular, this result implies that for any $\lambda>1$ and $t \geq 0$,

$$
p(\lambda t) \leq \sum_{j=2}^{M}\left|a_{j}\right| \lambda^{j} t^{j} \leq \lambda^{M} \sum_{j=2}^{M}\left|a_{j}\right| t^{j} \leq \frac{\lambda^{M}}{C_{M}} p(t) .
$$

Given a point $\boldsymbol{x}$ on the sphere of radius $A$ centered at the origin, we will let

$$
p(t)=g(t \boldsymbol{x}) .
$$

Notice that this defines a convex polynomial of one variable for which the bounds in equation (12) apply. Taking $t=1$ and $\lambda=\frac{B}{A}$ (where $A$ and $B$ are the radius of the inner and outer ball respectively) in equation (13), we have that

$$
p(1) \geq \frac{C_{M}}{\lambda^{M}} p\left(\frac{B}{A}\right)
$$

That is,

$$
g(\boldsymbol{x}) \geq C_{M} \frac{A^{M}}{B^{M}} g\left(\frac{B \boldsymbol{x}}{A}\right) .
$$

Since $|\boldsymbol{x}|=A$, then $\left|\frac{B}{A} \boldsymbol{x}\right|=B$. Because $g\left(\frac{B \boldsymbol{x}}{A}\right) \geq 1$ by assumption, it follows that $g(\boldsymbol{x}) \geq C_{M} \frac{A^{M}}{B^{M}}$. This completes the proof of Corollary 3.3.

Remark 3.5. Notice that the convexity of $g$ implies that

$$
g(\boldsymbol{x}) \geq C_{1} \geq C_{2} \sum_{\alpha}\left|c_{\alpha}\right|
$$

for any point $\boldsymbol{x}$ such that $|\boldsymbol{x}| \geq A$. 


\section{DECAY OF $\theta(\boldsymbol{\eta})$}

In this section we study the decay of a function $\theta(\boldsymbol{\eta})$, which we will presently define. The decay properties obtained for $\theta(\boldsymbol{\eta})$, stated in Lemma 4.1, will be used in the next section to study the decay of the denominator integral in the integral formula for the Szegö kernel derived in Proposition 2.1.

Lemma 4.1. Let

$$
g(\boldsymbol{v})=\sum_{\alpha \in \Gamma} c_{\alpha} \boldsymbol{v}^{\alpha}
$$

be a strictly convex polynomial in $\mathbb{R}^{n}$ such that

i) $g(\mathbf{0})=0$

ii) $\nabla g(\mathbf{0})=0$;

iii) there exists a constant $0<A<1$ such that $\{\boldsymbol{v}:|\boldsymbol{v}| \leq A\} \subseteq\{\boldsymbol{v}: g(\boldsymbol{v}) \leq 1\} \subseteq\{\boldsymbol{v}:|\boldsymbol{v}| \leq 1\}$; and

iv) there exist positive integers $m_{1}, \ldots, m_{n}$ such that the combined degree of $g$ is $\left(m_{1}, \ldots, m_{n}\right)$ (refer to definition on page 2).

Then

$$
\theta(\boldsymbol{\eta})=\left[\int_{\mathbb{R}^{n}} e^{\boldsymbol{\eta} \cdot \boldsymbol{v}-g(\boldsymbol{v})} d \boldsymbol{v}\right]^{-1}
$$

is a Schwartz function. Moreover, its decay depends only on the constant $A$ and the exponents $\left\{m_{1}, \ldots, m_{n}\right\}$.

Remark 4.2. Notice that under these assumptions, the coefficients of the polynomial $g(\boldsymbol{v})=\sum_{\alpha \in \Gamma} c_{\alpha} \boldsymbol{v}^{\alpha}$ satisfy $\sum_{\alpha \in \Gamma}\left|c_{\alpha}\right| \leq C$, where $C$ depends only on the constant $A$, on the degree of the polynomial and on the dimension of the space. This was shown in Corollary 3.3 on page 9.

Let $I=\int_{\mathbb{R}^{n}} e^{\boldsymbol{\eta} \cdot \boldsymbol{v}-g(\boldsymbol{v})} d \boldsymbol{v}$. We will show that $I$ grows at an exponential rate. We can write

$$
I=e^{h\left(\boldsymbol{v}_{\mathbf{0}}\right)} \int_{\mathbb{R}^{n}} e^{h(\boldsymbol{v})-h\left(\boldsymbol{v}_{\mathbf{0}}\right)} d \boldsymbol{v},
$$

where $h(\boldsymbol{v})=\boldsymbol{\eta} \cdot \boldsymbol{v}-g(\boldsymbol{v})$ and $\boldsymbol{v}_{\mathbf{0}}$ is the point where $h(\boldsymbol{v})$ attains its maximum (notice that $\boldsymbol{\eta}=$ $\left.\nabla g\left(\boldsymbol{v}_{\mathbf{0}}\right)\right)$. Notice that from the growth condition of $g$, and from the fact that $g$ is positive, it follows that $h$ is bounded above, and therefore attains a maximum. The convexity hypothesis further ensures that $h$ is strictly concave, and therefore that the maximum, $\boldsymbol{v}_{\mathbf{0}}$, is unique.

Notice that $h\left(\boldsymbol{v}_{\mathbf{0}}\right)=L(\boldsymbol{\eta})=\sup _{\boldsymbol{v}}\{\boldsymbol{\eta} \cdot \boldsymbol{v}-g(\boldsymbol{v})\}$ is the Legendre Transform of $g$. We will show that the dominant term, $e^{h\left(\boldsymbol{v}_{\mathbf{0}}\right)}$, grows at an exponential rate in $\boldsymbol{\eta}$. This term will provide the desired decay for $I^{-1}$. We will then show that $\int e^{h(\boldsymbol{v})-h\left(\boldsymbol{v}_{\mathbf{0}}\right)} d \boldsymbol{v}$ does not decrease too fast, that is, that it does not annul the growth of the dominant term. 
4.1. The dominant term. We begin by studying the growth of the term $e^{h\left(\boldsymbol{v}_{\mathbf{0}}\right)}=e^{L(\boldsymbol{\eta})}$. We show that $e^{L(\boldsymbol{\eta})}$ grows exponentially as a function of $\boldsymbol{\eta}$. Moreover, we claim that the growth is independent of the choice of $g$, but rather depends only on the constant $A$, on the combined degree of $g$ and on the dimension of the space. More precisely, we show that there exist positive constants $C, \widetilde{C}$ which depend only on the combined degree of $g$, the dimension of the space and the constant $A$, such that

$$
e^{L(\boldsymbol{\eta})} \geq \exp \left[\widetilde{C}\left(\left|\eta_{1}\right|^{\frac{2 m_{1}}{2 m_{1}-1}}+\ldots+\left|\eta_{n}\right|^{\frac{2 m_{n}}{2 m_{n}-1}}\right)-C\right] .
$$

We begin by showing that the polynomial $g$ is dominated, independently of its coefficients, by its pure terms of highest order.

Claim 4.3. If $g(\boldsymbol{v})$ is as in the statement of Lemma 4.1, then there exists a constant $C>0$ that depends only on the constant $A$, on the combined degree of the polynomial and on the dimension of the space such that

$$
g(\boldsymbol{v}) \leq C\left(1+v_{1}^{2 m_{1}}+\ldots+v_{n}^{2 m_{n}}\right) .
$$

Proof. Let

$$
r(\boldsymbol{v})=v_{1}^{2 m_{1}}+\ldots+v_{n}^{2 m_{n}}
$$

Notice that for any $\boldsymbol{v} \in \mathbb{R}^{n}$,

$$
v_{1}^{\alpha_{1}} \cdots v_{n}^{\alpha_{n}} \leq r(\boldsymbol{v})^{\frac{\alpha_{1}}{2 m_{1}}+\cdots+\frac{\alpha_{n}}{2 m_{n}}}
$$

Also, since $g \geq 0$,

$$
g(\boldsymbol{v})=|g(\boldsymbol{v})| \leq \sum_{\alpha \in \Gamma}\left|c_{\alpha}\right|\left|v_{1}^{\alpha_{1}} \cdots v_{n}^{\alpha_{n}}\right| \leq \sum_{\alpha \in \Gamma}\left|c_{\alpha}\right|\left|r(\boldsymbol{v})^{\frac{\alpha_{1}}{2 m_{1}}+\cdots+\frac{\alpha_{n}}{2 m_{n}}}\right|
$$

Moreover, recall that since $g$ is of combined degree $\left(m_{1}, \ldots, m_{n}\right)$, any index $\alpha \in \Gamma$ satisfies that

Hence,

$$
\frac{\alpha_{1}}{2 m_{1}}+\ldots+\frac{\alpha_{n}}{2 m_{n}} \leq 1
$$

$$
r(\boldsymbol{v})^{\frac{\alpha_{1}}{2 m_{1}}+\cdots+\frac{\alpha_{n}}{2 m_{n}}} \leq 1+r(\boldsymbol{v}) .
$$

Thus, and since $\sum_{\alpha \in \Gamma}\left|c_{\alpha}\right| \leq C$, it follows from equations (19) and (20) that

$$
g(\boldsymbol{v}) \leq \sum_{\alpha \in \Gamma}\left|c_{\alpha}\right|(1+r(\boldsymbol{v})) \leq C(1+r(\boldsymbol{v})) .
$$

This finishes the proof of Claim 4.3

Since this estimate does not depend on the coefficients of $g$, it is now easy to obtain a lower bound for $h\left(\boldsymbol{v}_{\mathbf{0}}\right)$ in terms of $\boldsymbol{\eta}$ which does not depend on the choice of $g$. 
AN APPLICATION OF JOHN ELLIPSOIDS TO THE SZEGŐ KERNEL ON UNBOUNDED CONVEX DOMAINS 13

Claim 4.4. The Legendre Transform of $g(\boldsymbol{v})$ where $\boldsymbol{v} \in \mathbb{R}^{n}$ is large for large values of $|\boldsymbol{\eta}|$. More precisely,

$$
L(\boldsymbol{\eta}) \geq \widetilde{C}\left(\left|\eta_{1}\right|^{\frac{2 m_{1}}{2 m_{1}-1}}+\ldots+\left|\eta_{n}\right|^{\frac{2 m_{n}}{2 m_{n}-1}}\right)-C,
$$

where $C$ and $\widetilde{C}$ are positive constants that depend only on the constant $A$, on the combined degree of $g$ and on the dimension of the space.

Proof. It follows from the previous claim that

$$
\begin{aligned}
L(\boldsymbol{\eta}) & =\sup _{\boldsymbol{v}}\{\boldsymbol{\eta} \cdot \boldsymbol{v}-g(\boldsymbol{v})\} \\
& \geq \sup _{\boldsymbol{v}}\left\{\boldsymbol{\eta} \cdot \boldsymbol{v}-C-C\left|v_{1}\right|^{2 m_{1}}-\ldots-C\left|v_{n}\right|^{2 m_{n}}\right\} \\
& =-C+\sup _{v_{1}}\left\{\eta_{1} v_{1}-C\left|v_{1}\right|^{2 m_{1}}\right\}+\ldots+\sup _{v_{n}}\left\{\eta_{n} v_{n}-C\left|v_{n}\right|^{2 m_{n}}\right\}
\end{aligned}
$$

But given $w \in \mathbb{R}$, the Legendre Transform of $\frac{B}{2 k}|w|^{2 k}$ is $\widetilde{B}|\eta|^{\frac{2 k}{2 k-1}}$, where

$$
\widetilde{B}=B^{\frac{-1}{2 k-1}}\left(\frac{2 k-1}{2 k}\right) \text {. }
$$

Thus,

$$
L(\boldsymbol{\eta}) \geq \widetilde{C}\left(\left|\eta_{1}\right|^{\frac{2 m_{1}}{2 m_{1}-1}}+\ldots+\left|\eta_{n}\right|^{\frac{2 m_{n}}{2 m_{n}-1}}\right)-C,
$$

where $\widetilde{C}=\min \left\{B_{1}^{\frac{-1}{2 m_{1}-1}}\left(\frac{2 m_{1}-1}{2 m_{1}}\right), \ldots, B_{n}^{\frac{-1}{2 m_{n}-1}}\left(\frac{2 m_{n}-1}{2 m_{n}}\right)\right\}$, and $B_{j}=C 2 m_{j}$.

This finishes the proof that the dominant term, $e^{L(\boldsymbol{\eta})}$, grows at an exponential rate in $\boldsymbol{\eta}$, independently of the coefficients of $g$. More precisely, we have shown that

$$
e^{L(\boldsymbol{\eta})} \geq \exp \left[\widetilde{C}\left(\left|\eta_{1}\right|^{\frac{2 m_{1}}{2 m_{1}-1}}+\ldots+\left|\eta_{n}\right|^{\frac{2 m_{n}}{2 m_{n}-1}}\right)-C\right] .
$$

4.2. A polynomial bound for the remaining terms. It suffices now to show that

$$
J=\int_{\mathbb{R}^{n}} e^{h(\boldsymbol{v})-h\left(\boldsymbol{v}_{\mathbf{0}}\right)} d \boldsymbol{v}
$$

is not too small to obtain the desired decay for $I^{-1}$. Recall that

$$
J=\int_{\mathbb{R}^{n}} e^{\boldsymbol{\eta} \cdot\left(\boldsymbol{v}-\boldsymbol{v}_{\mathbf{0}}\right)+g\left(\boldsymbol{v}_{\mathbf{0}}\right)-g(\boldsymbol{v})} d \boldsymbol{v} .
$$

In order to estimate this integral, we will use the fact that if $f: \mathbb{R}^{n} \rightarrow \mathbb{R}$ is a convex function such that $f(\mathbf{0})=0$ and $\nabla f(\mathbf{0})=0$ then (see Appendix)

$$
\int_{\mathbb{R}^{n}} e^{-f(\boldsymbol{w})} d \boldsymbol{w} \approx|\{\boldsymbol{w}: f(\boldsymbol{w}) \leq 1\}|
$$

Since $\boldsymbol{\eta}=\nabla g\left(\boldsymbol{v}_{\mathbf{0}}\right)$, and making the change of variables $\boldsymbol{w}=\boldsymbol{v}-\boldsymbol{v}_{\mathbf{0}}$, we can write 


$$
J=\int_{\mathbb{R}^{n}} e^{-f(\boldsymbol{w})} d \boldsymbol{w}
$$

where

$$
f(\boldsymbol{w})=-\nabla g\left(\boldsymbol{v}_{\mathbf{0}}\right) \cdot \boldsymbol{w}-g\left(\boldsymbol{v}_{\mathbf{0}}\right)+g\left(\boldsymbol{v}_{\mathbf{0}}+\boldsymbol{w}\right) .
$$

Clearly $f(\mathbf{0})=0$ and $\nabla f(\mathbf{0})=0$. Also, since $g$ is convex, so is $f$. Thus,

$$
J \approx|\{\boldsymbol{w}: f(\boldsymbol{w}) \leq 1\}|
$$

That is,

$$
I=\int_{\mathbb{R}^{n}} e^{\eta \cdot \boldsymbol{v}-g(\boldsymbol{v})} d \boldsymbol{v} \approx e^{h\left(\boldsymbol{v}_{\mathbf{0}}\right)}|\{\boldsymbol{w}: f(\boldsymbol{w}) \leq 1\}|
$$

Our goal is to show that as $|\boldsymbol{\eta}|$ grows, the volume given in equation (23) decreases slower than the rate of growth we obtained for $e^{h\left(\boldsymbol{v}_{\mathbf{0}}\right)}$. We begin by obtaining an upper bound for $f$ that is independent of the choice of $g$, but rather depends only on its combined degree, on the dimension of the space and on the constant $A$ (where the constant $A$ is from assumption (iii) of Lemma 4.1). To do so we will write $f$ as an integral in terms of the quadratic form associated to the Hessian of $g$. In Claim 4.5 we obtain an upper bound for this quadratic form in terms of a polynomial that is independent of the coefficients of $g$. In Claim 4.6 we use this estimate to obtain the desired bound for $f$.

Claim 4.5. There is a constant $C$ depending only on the combined degree of $g$ so that

$$
\sum_{i, j=1}^{n} g_{i j}(\boldsymbol{v}) w_{i} w_{j} \leq C(1+r(\boldsymbol{v}))|\boldsymbol{w}|^{2},
$$

where $r(\boldsymbol{v})=v_{1}^{2 m_{1}}+\ldots+v_{n}^{2 m_{n}}$ and $g_{i j}(\boldsymbol{v})=\frac{\partial^{2} g}{\partial v_{i} \partial v_{j}}(\boldsymbol{v})$.

Proof. Let $L$ be the Hessian matrix of $g$ so that $\boldsymbol{w}^{\mathbf{T}} L \boldsymbol{w}=\sum_{i, j=1}^{n} g_{i j} w_{i} w_{j}$. Since $L$ is symmetric, it has $n$ linearly independent eigenvectors. Let $\boldsymbol{u}_{\boldsymbol{i}}, i=1, \ldots, n$ be the eigenvectors of $\mathrm{L}$, and $\lambda_{i}, i=1, \ldots, n$ be the corresponding eigenvalues. Since $g$ is convex, the matrix $L$ is positive semi-definite, so its eigenvalues are non-negative.

Let $P=\operatorname{Tr}(L) I$, where $\operatorname{Tr}(L)=\lambda_{1}+\ldots+\lambda_{n}$ is the trace of the matrix $L$ and $I$ is the identity matrix. Let $Q=P-L$. We claim that $Q$ is positive semi-definite, and hence that $L \leq P$ as quadratic forms. In fact, notice that for $i=1, \ldots, n$

$$
Q \boldsymbol{u}_{i}=P \boldsymbol{u}_{i}-L \boldsymbol{u}_{i}=\operatorname{Tr}(L) \boldsymbol{u}_{i}-\lambda_{i} \boldsymbol{u}_{i}=\left(\operatorname{Tr}(L)-\lambda_{i}\right) \boldsymbol{u}_{i}=\sum_{\substack{1 \leq j \leq n \\ j \neq i}} \lambda_{j} \boldsymbol{u}_{i} .
$$

Thus, for $i=1, \ldots, n, \boldsymbol{u}_{\boldsymbol{i}}$ is an eigenvector of $Q$, with eigenvalue

$$
\mu_{i}=\sum_{\substack{1 \leq j \leq n \\ j \neq i}} \lambda_{j}>0
$$


Thus, since $Q$ is a symmetric matrix whose eigenvalues are non-negative, $Q$ is positive semi-definite. Hence, since $\boldsymbol{w}^{\mathbf{T}} L \boldsymbol{w} \leq \boldsymbol{w}^{\mathbf{T}} P \boldsymbol{w}$, it follows that

$$
0 \leq \sum_{i, j=1}^{n} g_{i j}(\boldsymbol{v}) w_{i} w_{j} \leq\left(\left|g_{11}(\boldsymbol{v})\right|+\ldots+\left|g_{n n}(\boldsymbol{v})\right|\right)|w|^{2}
$$

Notice that each $g_{j j}(\boldsymbol{v})$ is a polynomial of the form $\sum_{\beta} \widetilde{c_{\beta}} \boldsymbol{v}^{\beta}$ where the indexes $\beta$ satisfy

$$
\frac{\beta_{1}}{2 m_{1}}+\cdots+\frac{\beta_{n}}{2 m_{n}} \leq 1-\frac{1}{m_{j}}<1
$$

In particular, this implies that

$$
r(\boldsymbol{v})^{\frac{\beta_{1}}{2 m_{1}}+\cdots+\frac{\beta_{n}}{2 m_{n}}} \leq 1+r(\boldsymbol{v}) .
$$

Moreover, since for any $\boldsymbol{v} \in \mathbb{R}^{n}$ we have that $v_{1}^{\beta_{1}} \cdots v_{n}^{\beta_{n}} \leq r(\boldsymbol{v})^{\frac{\beta_{1}}{2 m_{1}}+\cdots+\frac{\beta_{n}}{2 m_{n}}}$ it follows that

$$
\left|g_{j j}(\boldsymbol{v})\right| \leq \sum_{\beta}\left|\widetilde{c_{\beta}}\right|\left|v_{1}^{\beta_{1}} \cdots v_{n}^{\beta_{n}}\right| \leq \sum_{\beta}\left|\widetilde{c_{\beta}}\right|(1+r(\boldsymbol{v}))
$$

Since $\sum_{\alpha \in \Gamma}\left|c_{\alpha}\right| \leq C$, the sum $\sum_{\beta \in \Gamma} \widetilde{c_{\beta}}$ is also bounded by a constant that does not depend on the choice of $g$, but rather on the combined degree of $g$. Then, and by the previous inequality, we have that

$$
\left|g_{11}(\boldsymbol{v})\right|+\ldots+\left|g_{n n}(\boldsymbol{v})\right| \leq C(1+r(\boldsymbol{v})),
$$

where $C$ depends only on the combined degree of $g$. That is,

$$
\sum_{i, j=1}^{n} g_{i j}(\boldsymbol{v}) w_{i} w_{j} \leq C(1+r(\boldsymbol{v}))|\boldsymbol{w}|^{2} .
$$

Using this result it is now possible to obtain an upper bound for $f$ which is independent of the choice of $g$. We do so in the following claim.

Claim 4.6. If

$$
r(\boldsymbol{v})=v_{1}^{2 m_{1}}+\ldots+v_{n}^{2 m_{n}}
$$

and

$$
f(\boldsymbol{w})=-\nabla g\left(\boldsymbol{v}_{\mathbf{0}}\right) \cdot \boldsymbol{w}-g\left(\boldsymbol{v}_{\mathbf{0}}\right)+g\left(\boldsymbol{v}_{\mathbf{0}}+\boldsymbol{w}\right)
$$

then,

$$
f(\boldsymbol{w}) \lesssim|\boldsymbol{w}|^{2}\left(1+r\left(\boldsymbol{v}_{\mathbf{0}}\right)+r(\boldsymbol{w})\right),
$$

where the constant depends only on the constant $A$, the combined degree of $g$ and the dimension of the space. 
Proof. We begin by rewriting $f$ as an integral in terms of the quadratic form associated to the Hessian, so that we can apply our previous estimate. Integrating by parts, we can write

$$
g\left(\boldsymbol{v}_{\mathbf{0}}+\boldsymbol{w}\right)-g\left(\boldsymbol{v}_{\mathbf{0}}\right)=\nabla g\left(\boldsymbol{v}_{\mathbf{0}}\right) \cdot \boldsymbol{w}+\int_{0}^{1} \sum_{i, j=1}^{n} g_{i j}\left(\boldsymbol{v}_{\mathbf{0}}+t \boldsymbol{w}\right) w_{i} w_{j}(1-t) d t
$$

It follows that

$$
f(\boldsymbol{w})=\int_{0}^{1} \sum_{i, j=1}^{n} g_{i j}\left(\boldsymbol{v}_{\mathbf{0}}+t \boldsymbol{w}\right) w_{i} w_{j}(1-t) d t .
$$

In particular, by convexity of $g$ we have that $f \geq 0$.

We can now use the bound for the Hessian obtained in Claim 4.5. It follows that

$$
f(\boldsymbol{w}) \lesssim \int_{0}^{1}\left(1+r\left(\boldsymbol{v}_{\mathbf{0}}+t \boldsymbol{w}\right)\right)|\boldsymbol{w}|^{2}(1-t) d t
$$

Using convexity, it is easy to show that $r(\boldsymbol{u}+\boldsymbol{v}) \leq \max \left\{2^{2 m_{1}-1}, \ldots, 2^{2 m_{n}-1}\right\}[r(\boldsymbol{u})+r(\boldsymbol{v})]$. Applying this inequality to $r\left(\boldsymbol{v}_{\mathbf{0}}+t \boldsymbol{w}\right)$, and since $0 \leq t \leq 1$, we have that

$$
r\left(\boldsymbol{v}_{\mathbf{0}}+t \boldsymbol{w}\right) \lesssim r\left(\boldsymbol{v}_{\mathbf{0}}\right)+r(t \boldsymbol{w}) \leq r\left(\boldsymbol{v}_{\mathbf{0}}\right)+r(\boldsymbol{w}) .
$$

Hence, it follows from equation (26) that

$$
f(\boldsymbol{w}) \lesssim|\boldsymbol{w}|^{2}\left(1+r\left(\boldsymbol{v}_{\mathbf{0}}\right)+r(\boldsymbol{w})\right)
$$

In the next three claims we show that there is a polynomial $P$ and a constant $C$ depending only on the degrees $\left\{m_{1}, \ldots, m_{n}\right\}$ so that

$$
|\{\boldsymbol{w}: f(\boldsymbol{w}) \leq 1\}|^{-1} \leq C(1+P(|\boldsymbol{\eta}|))^{\frac{n}{2}} .
$$

In Claim 4.7 we show that $|\{\boldsymbol{w}: f(\boldsymbol{w}) \leq 1\}|^{-1}$ is bounded by a polynomial in terms of $r\left(\boldsymbol{v}_{\mathbf{0}}\right)$, and in Claim 4.8 we compare the sizes of $\left|\boldsymbol{v}_{\mathbf{0}}\right|$ and $|\boldsymbol{\eta}|$. In Claim 4.9 we conclude that $r\left(\boldsymbol{v}_{\mathbf{0}}\right)$ grows at most at a polynomial rate in $|\boldsymbol{\eta}|$.

Claim 4.7. If $f(\boldsymbol{w}) \lesssim|\boldsymbol{w}|^{2}\left(1+r\left(\boldsymbol{v}_{\mathbf{0}}\right)+r(\boldsymbol{w})\right)$, then

$$
|\{\boldsymbol{w}: f(\boldsymbol{w}) \leq 1\}| \gtrsim\left(1+r\left(\boldsymbol{v}_{\mathbf{0}}\right)\right)^{-\frac{n}{2}}
$$

Proof. Let $C$ be such that $f(\boldsymbol{w}) \leq C|\boldsymbol{w}|^{2}\left(1+r\left(\boldsymbol{v}_{\mathbf{0}}\right)+r(\boldsymbol{w})\right)$ and let

$$
T(\boldsymbol{w})=C|\boldsymbol{w}|^{2}\left(1+r\left(\boldsymbol{v}_{\mathbf{0}}\right)+r(\boldsymbol{w})\right)
$$

Then,

$$
|\{\boldsymbol{w}: f(\boldsymbol{w}) \leq 1\}| \geq|\{\boldsymbol{w}: T(\boldsymbol{w}) \leq 1\}|
$$


Let $\Sigma=\{\boldsymbol{u}: T(\boldsymbol{u})=1\}$ and let $m=\min \{|\boldsymbol{u}|: \boldsymbol{u} \in \Sigma\}$. Choose $\boldsymbol{w}_{\boldsymbol{T}}$ such that $T\left(\boldsymbol{w}_{\boldsymbol{T}}\right)=1$ and $\left|\boldsymbol{w}_{T}\right|=m$. Then the set $|\{\boldsymbol{w}: T(\boldsymbol{w}) \leq 1\}|$ is bounded from below by the volume of the ball of radius $\left|\boldsymbol{w}_{T}\right|$. That is,

where $C_{n}=\frac{\pi^{\frac{n}{2}}}{\Gamma\left(\frac{n}{2}+1\right)}$.

$$
|\{\boldsymbol{w}: T(\boldsymbol{w}) \leq 1\}| \geq C_{n}\left|\boldsymbol{w}_{\boldsymbol{T}}\right|^{n}
$$

Let $a=1+r\left(\boldsymbol{v}_{\mathbf{0}}\right)$. Our goal is to show that $\left|\boldsymbol{w}_{\boldsymbol{T}}\right|^{n} \gtrsim a^{-\frac{n}{2}}$. If $\left|\boldsymbol{w}_{\boldsymbol{T}}\right|^{2} \geq \frac{1}{2 C a}$, then $\left|\boldsymbol{w}_{\boldsymbol{T}}\right|^{n} \gtrsim a^{-\frac{n}{2}}$, as desired. Otherwise, we have that $\left|\boldsymbol{w}_{\boldsymbol{T}}\right|^{2}<\frac{1}{2 C a}$. Since $1=T\left(\boldsymbol{w}_{\boldsymbol{T}}\right)=C\left|\boldsymbol{w}_{\boldsymbol{T}}\right|^{2}\left(1+r\left(\boldsymbol{v}_{\mathbf{0}}\right)+r\left(\boldsymbol{w}_{\boldsymbol{T}}\right)\right)$ it follows that

$$
a\left|\boldsymbol{w}_{\boldsymbol{T}}\right|^{2}+\left|\boldsymbol{w}_{\boldsymbol{T}}\right|^{2} r\left(\boldsymbol{w}_{\boldsymbol{T}}\right)=\frac{1}{C}
$$

But since $\left|\boldsymbol{w}_{\boldsymbol{T}}\right|^{2}<\frac{1}{2 C a}$, it follows that

$$
\frac{1}{2 C}<\left|\boldsymbol{w}_{\boldsymbol{T}}\right|^{2} r\left(\boldsymbol{w}_{\boldsymbol{T}}\right)
$$

Also, since $a \geq 1$, we have that $\left|\boldsymbol{w}_{T}\right|^{2}<\frac{1}{2 C}$. Thus, $w_{T j}^{2}<\frac{1}{2 C}$ for every $1 \leq j \leq n$. Hence,

$$
r\left(\boldsymbol{w}_{\boldsymbol{T}}\right)<\left(\frac{1}{2 C}\right)^{m_{1}}+\cdots+\left(\frac{1}{2 C}\right)^{m_{n}} .
$$

Using this in equation (27) we have that

$$
\frac{1}{2 C}<\left|\boldsymbol{w}_{\boldsymbol{T}}\right|^{2}\left(\left(\frac{1}{2 C}\right)^{m_{1}}+\cdots+\left(\frac{1}{2 C}\right)^{m_{n}}\right)
$$

Thus, and since $a \geq 1$, it follows that

$$
\left|\boldsymbol{w}_{T}\right|^{2}>\Lambda \geq \frac{\Lambda}{a}
$$

where $\Lambda=\frac{1}{2 C}\left(\left(\frac{1}{2 C}\right)^{m_{1}}+\cdots+\left(\frac{1}{2 C}\right)^{m_{n}}\right)^{-1}$ is a strictly positive constant.

Therefore,

$$
|\{\boldsymbol{w}: f(\boldsymbol{w}) \leq 1\}| \gtrsim\left|\boldsymbol{w}_{T}\right|^{n} \gtrsim a^{-\frac{n}{2}} .
$$

This finishes the proof of Claim 4.7.

Claim 4.8. There exist positive constants $\beta_{1}$ and $\beta_{2}$ such that

$$
\left|\boldsymbol{v}_{\mathbf{0}}\right| \leq \beta_{1}|\boldsymbol{\eta}|+\beta_{2} .
$$

The constants depend only on $m_{1}, \ldots, m_{n}$ and the dimension of the space.

In the proof of Claim 4.8, we use Lemma 2.2 of [5]. For the reader's convenience, we state the Lemma below. 
Lemma 2.2 (Bruna - Nagel - Wainger). Let $C(m, T)$ denote the space of polynomials

which satisfy:

$$
P(t)=\sum_{j=0}^{m} a_{j} t^{j}
$$

(a) The degree of $P$ is no bigger than $m$;

(b) $P(0)=a_{0}=0 ; P^{\prime}(0)=a_{1}=0$;

(c) $P$ is convex for $0 \leq t \leq T$.

Then there is a constant $C_{m}$, independent of $T$, so that if $P \in C(m, T), P(t)=\sum_{j=2}^{m} a_{j} t^{j}$, then

$$
P^{\prime}(t) \geq C_{m} \sum_{j=2}^{m}\left|a_{j}\right| t^{j-1}
$$

for $0 \leq t \leq T$. In particular,

$$
\begin{array}{ll}
P^{\prime}(t) \geq C_{m} t^{m-1} \sum_{j=2}^{m}\left|a_{j}\right| & \text { if } 0 \leq t \leq 1, \quad \text { and } \\
P^{\prime}(t) \geq C_{m} t \sum_{j=2}^{m}\left|a_{j}\right| & \text { if } 1 \leq t \leq T .
\end{array}
$$

Proof of Claim 4.8. The statement is trivial if $\left|\boldsymbol{v}_{\mathbf{0}}\right| \leq 1$, so we will assume that $\left|\boldsymbol{v}_{\mathbf{0}}\right|>1$. Let $G(t)=$ $g\left(\frac{t v_{0}}{\left|\boldsymbol{v}_{\mathbf{0}}\right|}\right)$. Then

$$
G^{\prime}(t)=\nabla g\left(\frac{t \boldsymbol{v}_{\mathbf{0}}}{\left|\boldsymbol{v}_{\mathbf{0}}\right|}\right) \cdot\left(\frac{\boldsymbol{v}_{\mathbf{0}}}{\left|\boldsymbol{v}_{\mathbf{0}}\right|}\right) .
$$

Thus, since $\nabla g(\mathbf{0})=\mathbf{0}$ by hypothesis, $G^{\prime}(0)=\nabla g(\mathbf{0}) \cdot\left(\frac{\boldsymbol{v}_{\mathbf{0}}}{\left|\boldsymbol{v}_{\mathbf{0}}\right|}\right)=0$. Also, notice that since $g$ is convex, so is $G$. Hence, and since $G$ is a polynomial, $G^{\prime}(t)>0$ if $t>0$. By Cauchy-Schwarz,

$$
\left|G^{\prime}(t)\right| \leq\left|\nabla g\left(\frac{t \boldsymbol{v}_{\mathbf{0}}}{\left|\boldsymbol{v}_{\mathbf{0}}\right|}\right)\right|
$$

Evaluating at $t=\left|\boldsymbol{v}_{\mathbf{0}}\right|$ we have that $\left|G^{\prime}\left(\left|\boldsymbol{v}_{\mathbf{0}}\right|\right)\right| \leq\left|\nabla g\left(\boldsymbol{v}_{\mathbf{0}}\right)\right|=|\boldsymbol{\eta}|$. But since $\left|\boldsymbol{v}_{\mathbf{0}}\right|>0$, it follows that

$$
G^{\prime}\left(\left|\boldsymbol{v}_{\mathbf{0}}\right|\right)=\left|G^{\prime}\left(\left|\boldsymbol{v}_{\mathbf{0}}\right|\right)\right| \leq|\boldsymbol{\eta}|
$$

It suffices now to obtain a polynomial lower bound for $G^{\prime}\left(\left|\boldsymbol{v}_{\mathbf{0}}\right|\right)$ in terms of $\left|\boldsymbol{v}_{\mathbf{0}}\right|$. To do so, we use Lemma 2.2 of [5].

Notice that $G(t)$ is a convex polynomial of one variable such that $G(0)=G^{\prime}(0)=0$, so we can use the aforementioned result. Write

$$
G(t)=\sum_{j=2}^{m} a_{j} t^{j}
$$

Since we are considering $\left|\boldsymbol{v}_{\mathbf{0}}\right|>1$, it follows from equations (29) and (28) that

$$
\left|\boldsymbol{v}_{\mathbf{0}}\right| \sum_{j=2}^{m}\left|a_{j}\right| \lesssim G^{\prime}\left(\left|\boldsymbol{v}_{\mathbf{0}}\right|\right) \leq|\boldsymbol{\eta}|
$$


It suffices now to obtain a lower bound for $\sum_{j=2}^{m}\left|a_{j}\right|$, which must be independent of the choice of $g$. To do so, we use assumption (iii) of Lemma 4.1, namely, the fact that

$$
\{\boldsymbol{v}: g(\boldsymbol{v}) \leq 1\} \subseteq\{\boldsymbol{v}:|\boldsymbol{v}| \leq 1\} .
$$

In particular, if $|\boldsymbol{v}|=1$, it must follow that $g(\boldsymbol{v}) \geq 1$. Thus, evaluating at $t=1$, it follows that

$$
G(1)=g\left(\frac{\boldsymbol{v}_{\mathbf{0}}}{\left|\boldsymbol{v}_{\mathbf{0}}\right|}\right) \geq 1
$$

But $G(1)=\sum_{j=2}^{m} a_{j} \leq \sum_{j=2}^{m}\left|a_{j}\right|$. Using this bound on equation (30) yields $\left|\boldsymbol{v}_{\mathbf{0}}\right| \lesssim|\boldsymbol{\eta}|$. This finishes the proof of Claim 4.8.

Claim 4.9. For $p \in \mathbb{R},\left(1+r\left(\boldsymbol{v}_{\mathbf{0}}\right)^{p}\right)^{\frac{n}{2}}$ is at most of polynomial growth in $|\boldsymbol{\eta}|$.

Proof. The proof is trivial. In fact, since $\left|\boldsymbol{v}_{\mathbf{0}}\right| \leq \beta_{1}|\boldsymbol{\eta}|+\beta_{2}$, it is clear that $r\left(\boldsymbol{v}_{\mathbf{0}}\right)=v_{01}^{2 m_{1}}+\ldots+v_{0 n}^{2 m_{n}} \leq$ $\left|\boldsymbol{v}_{\mathbf{0}}\right|^{2 m_{1}}+\ldots+\left|\boldsymbol{v}_{\mathbf{0}}\right|^{2 m_{n}}$ is bounded from above by a polynomial in $|\boldsymbol{\eta}|$.

It follows from these claims that there exists a polynomial $P(|\boldsymbol{\eta}|)$, which does not depend on the choice of $g$, such that

$$
\theta(\boldsymbol{\eta})=I^{-1} \lesssim \exp \left[-C\left(\left|\eta_{1}\right|^{\frac{2 m_{1}}{2 m_{1}-1}}+\ldots+\left|\eta_{n}\right|^{\frac{2 m_{n}}{2 m_{n}-1}}\right)\right](1+P(|\boldsymbol{\eta}|))^{\frac{n}{2}}
$$

This finishes the proof that $\theta(\boldsymbol{\eta})$ decays at an exponential rate. Moreover, this decay is independent of the coefficients of the polynomial $g$ that defines it. We must now show that the same is true of all the derivatives of $\theta(\boldsymbol{\eta})$.

4.3. Decay of the derivatives. The derivatives of $\theta(\boldsymbol{\eta})$ consist of sums of terms of the form

$$
\frac{C\left[\int_{\mathbb{R}^{n}} e^{\boldsymbol{\eta} \boldsymbol{v}-g(\boldsymbol{v})} v_{1}^{i_{1,1}} \cdots v_{n}^{i_{n, 1}} d \boldsymbol{v}\right]^{a_{1}} \cdots\left[\int_{\mathbb{R}^{n}} e^{\boldsymbol{\eta} \boldsymbol{v}-g(\boldsymbol{v})} v_{1}^{i_{1, r}} \cdots v_{n}^{i_{n, r}} d \boldsymbol{v}\right]^{a_{r}}}{\left[\int_{\mathbb{R}^{n}} e^{\boldsymbol{\eta} \boldsymbol{v}-g(\boldsymbol{v})} d \boldsymbol{v}\right]^{d}},
$$

where $i_{1,1}, \ldots, i_{n, r}, a_{1}, \ldots, a_{r}, d \in \mathbb{N}$ and $a_{1}+\ldots+a_{r}+1=d$. We show that each of these terms decays rapidly, and that the decay depends only on the coefficients of $g$.

Remark 4.10. The fact that $a_{1}+\ldots+a_{r}-d<0$ is crucial. As before (equation (16)), we can factor out a term $e^{h\left(\boldsymbol{v}_{\mathbf{0}}\right)}$ for each of these integrals. That is, we will factor out $\left(e^{h\left(\boldsymbol{v}_{\mathbf{0}}\right)}\right)^{a_{1}+\ldots+a_{r}-d}=e^{-h\left(\boldsymbol{v}_{\mathbf{0}}\right)}$. This term will provide the desired decay.

In order to understand the decay of the derivatives of $\theta(\boldsymbol{\eta})$ we need to study integrals of the form $\int_{\mathbb{R}^{n}} e^{\boldsymbol{\eta} \boldsymbol{v}-g(\boldsymbol{v})} v_{1}^{i_{1}} \cdots v_{n}^{i_{n}} d \boldsymbol{v}$. 


\section{Claim 4.11.}

$$
\left|\int_{\mathbb{R}^{n}} e^{\boldsymbol{\eta} \boldsymbol{v}-g(\boldsymbol{v})} v_{1}^{i_{1}} \cdots v_{n}^{i_{n}} d \boldsymbol{v}\right| \lesssim e^{h\left(\boldsymbol{v}_{\mathbf{0}}\right)} H_{f}\left[\left|\boldsymbol{v}_{\mathbf{0}}\right|\right]
$$

where

$$
\begin{aligned}
H_{f}\left[\left|\boldsymbol{v}_{\mathbf{0}}\right|\right] & =\sum_{s_{1}=0}^{i_{1}} \cdots \sum_{s_{n}=0}^{i_{n}}\left(\begin{array}{c}
i_{1} \\
s_{1}
\end{array}\right) \cdots\left(\begin{array}{c}
i_{n} \\
s_{n}
\end{array}\right)\left|\boldsymbol{v}_{\mathbf{0}}\right|^{i_{1}+\ldots+i_{n}-s} \\
& \times\left(|\{\boldsymbol{w}: f(\boldsymbol{w}) \leq 1\}|\left(1+\left|\boldsymbol{v}_{\mathbf{0}}\right|^{s B}\right)+\Theta\right) ;
\end{aligned}
$$

$\Theta$ is a constant that depends only on the combined degree of $g$ and the dimension of the space; $s=s_{1}+\cdots+s_{n} ;$ and $B=4 \max \left\{m_{1}, \ldots, m_{n}\right\}$.

Proof. As before (equation (16)), we can write

$$
\widetilde{I}=\int_{\mathbb{R}^{n}} e^{\boldsymbol{\eta} \boldsymbol{v}-g(\boldsymbol{v})} v_{1}^{i_{1}} \cdots v_{n}^{i_{n}} d \boldsymbol{v}=e^{h\left(\boldsymbol{v}_{\mathbf{0}}\right)} \int_{\mathbb{R}^{n}} e^{h(\boldsymbol{v})-h\left(\boldsymbol{v}_{\mathbf{0}}\right)} v_{1}^{i_{1}} \cdots v_{n}^{i_{n}} d \boldsymbol{v},
$$

where $h(\boldsymbol{v})=\boldsymbol{\eta} \cdot \boldsymbol{v}-g(\boldsymbol{v})$ and $\boldsymbol{v}_{\mathbf{0}}$ is the point where $h(\boldsymbol{v})$ attains its maximum; and

$$
\widetilde{J}=\int_{\mathbb{R}^{n}} e^{h(\boldsymbol{v})-h\left(\boldsymbol{v}_{\mathbf{0}}\right)} v_{1}^{i_{1}} \cdots v_{n}^{i_{n}} d \boldsymbol{v}=\int_{\mathbb{R}^{n}}\left(w_{1}+v_{01}\right)^{i_{1}} \cdots\left(w_{n}+v_{0 n}\right)^{i_{n}} e^{-f(\boldsymbol{w})} d \boldsymbol{w},
$$

where $f(\boldsymbol{w})=g\left(\boldsymbol{v}_{\mathbf{0}}+\boldsymbol{w}\right)-g\left(\boldsymbol{v}_{\mathbf{0}}\right)-\nabla g\left(\boldsymbol{v}_{\mathbf{0}}\right) \cdot \boldsymbol{w}$ as in equation (22). Writing

$$
\widetilde{J}=\sum_{s_{1}=0}^{i_{1}} \cdots \sum_{s_{n}=0}^{i_{n}}\left(\begin{array}{c}
i_{1} \\
s_{1}
\end{array}\right) \cdots\left(\begin{array}{c}
i_{n} \\
s_{n}
\end{array}\right) v_{01}^{i_{1}-s_{1}} \cdots v_{0 n}^{i_{n}-s_{n}} \int_{\mathbb{R}^{n}} w_{1}^{s_{1}} \cdots w_{n}^{s_{n}} e^{-f(\boldsymbol{w})} d \boldsymbol{w},
$$

it follows that

$$
|\widetilde{J}| \leq \sum_{s_{1}=0}^{i_{1}} \ldots \sum_{s_{n}=0}^{i_{n}}\left(\begin{array}{l}
i_{1} \\
s_{1}
\end{array}\right) \cdots\left(\begin{array}{l}
i_{n} \\
s_{n}
\end{array}\right)\left|\boldsymbol{v}_{\mathbf{0}}\right|^{i_{1}+\ldots+i_{n}-\left(s_{1}+\ldots+s_{n}\right)} \int_{\mathbb{R}^{n}}|\boldsymbol{w}|^{s_{1}+\ldots+s_{n}} e^{-f(\boldsymbol{w})} d \boldsymbol{w} .
$$

Let $s=s_{1}+\cdots+s_{n}$ and $J_{s}=\int_{\mathbb{R}^{n}}|\boldsymbol{w}|^{s} e^{-f(\boldsymbol{w})} d \boldsymbol{w}$. Write

$$
J_{s}=\int_{\left\{\boldsymbol{w} \in \mathbb{R}^{n}:|\boldsymbol{w}| \leq 1\right\}}|\boldsymbol{w}|^{s} e^{-f(\boldsymbol{w})} d \boldsymbol{w}+\int_{\left\{\boldsymbol{w} \in \mathbb{R}^{n}:|\boldsymbol{w}|>1\right\}}|\boldsymbol{w}|^{s} e^{-f(\boldsymbol{w})} d \boldsymbol{w}=J_{s_{1}}+J_{s_{2}} .
$$

Then

$$
J_{s_{1}} \leq \int_{\mathbb{R}^{n}} e^{-f(\boldsymbol{w})} d \boldsymbol{w} \approx|\{\boldsymbol{w}: f(\boldsymbol{w}) \leq 1\}| .
$$

Given $\boldsymbol{v}_{\mathbf{0}}$, we can estimate the size of $J_{s_{2}}$ by splitting the integral into the two following regions:

$$
J_{s_{2}}=\int_{\substack{|\boldsymbol{w}|>1 \\|\boldsymbol{w}| \leq \lambda\left|\boldsymbol{v}_{\mathbf{0}}\right|^{B}}}|\boldsymbol{w}|^{s} e^{-f(\boldsymbol{w})} d \boldsymbol{w}+\int_{\substack{|\boldsymbol{w}|>1 \\|\boldsymbol{w}|>\lambda\left|\boldsymbol{v}_{\mathbf{0}}\right|^{B}}}|\boldsymbol{w}|^{s} e^{-f(\boldsymbol{w})} d \boldsymbol{w},
$$

for some large constant $\lambda$ yet to be determined and $B=4 \max \left\{m_{1}, \ldots, m_{n}\right\}$. Then

$$
\int_{\substack{|\boldsymbol{w}|>1 \\|\boldsymbol{w}| \leq \lambda\left|\boldsymbol{v}_{\mathbf{0}}\right|^{B}}}|\boldsymbol{w}|^{s} e^{-f(\boldsymbol{w})} d \boldsymbol{w} \leq \lambda^{s}\left|\boldsymbol{v}_{\mathbf{0}}\right|^{s B} \int_{\mathbb{R}^{n}} e^{-f(\boldsymbol{w})} d \boldsymbol{w} \approx \lambda^{s}\left|\boldsymbol{v}_{\mathbf{0}}\right|^{s B}|\{\boldsymbol{w}: f(\boldsymbol{w}) \leq 1\}| .
$$


AN APPLICATION OF JOHN ELLIPSOIDS TO THE SZEGŐ KERNEL ON UNBOUNDED CONVEX DOMAINS 21

In order to estimate $\int_{\left\{\boldsymbol{w}:|\boldsymbol{w}|>1,|\boldsymbol{w}|>\lambda\left|\boldsymbol{v}_{\mathbf{0}}\right|^{B}\right\}}|\boldsymbol{w}|^{s} e^{-f(\boldsymbol{w})} d \boldsymbol{w}$, we will find a lower bound in this region for $f(\boldsymbol{w})$ in terms of $|\boldsymbol{w}|^{2}$ and we will then bound the integral by a constant. Since $f \geq 0$,

$$
f(\boldsymbol{w}) \geq\left|g\left(\boldsymbol{v}_{\mathbf{0}}+\boldsymbol{w}\right)\right|-\left|g\left(\boldsymbol{v}_{\mathbf{0}}\right)\right|-\left|\nabla g\left(\boldsymbol{v}_{\mathbf{0}}\right) \cdot \boldsymbol{w}\right|
$$

We will show that $g\left(\boldsymbol{v}_{\mathbf{0}}+\boldsymbol{w}\right)$ is bounded from below by a constant multiple of $|\boldsymbol{w}|^{2}$. It will then suffice to show that the remaining terms in the above expression can be dominated by this bound.

Let

$$
F(t)=g\left(\frac{t\left(\boldsymbol{v}_{\mathbf{0}}+\boldsymbol{w}\right)}{\left|\boldsymbol{v}_{\mathbf{0}}+\boldsymbol{w}\right|}\right)
$$

where $t \in \mathbb{R}$. Then $F(t)$ is a convex polynomial in one variable, such that $F(0)=F^{\prime}(0)=0$. We will write

$$
F(t)=\sum_{j=2}^{M} a_{j} t^{j}
$$

Notice that in the region we are considering, and since $B>1$, we have that

$$
\left|\boldsymbol{v}_{\mathbf{0}}+\boldsymbol{w}\right| \geq|\boldsymbol{w}|-\left|\boldsymbol{v}_{\mathbf{0}}\right|>|\boldsymbol{w}|-\frac{|\boldsymbol{w}|^{\frac{1}{B}}}{\lambda^{\frac{1}{B}}} \geq|\boldsymbol{w}|\left(1-\frac{1}{\lambda^{\frac{1}{B}}}\right)>1-\frac{1}{\lambda^{\frac{1}{B}}} .
$$

In particular, if $\lambda>2^{B}$, then $\left|\boldsymbol{v}_{\mathbf{0}}+\boldsymbol{w}\right|>1 / 2$. But it follows from Lemma 2.1 of [5] (refer to equation (12) on page 10), that there exists a constant $C_{M}>0$ that depends only on the degree of $F$ such that

$$
g\left(\boldsymbol{v}_{\mathbf{0}}+\boldsymbol{w}\right)=F\left(\left|\boldsymbol{v}_{\mathbf{0}}+\boldsymbol{w}\right|\right) \geq C_{M}\left|\boldsymbol{v}_{\mathbf{0}}+\boldsymbol{w}\right|^{2} \sum_{j=2}^{M}\left|a_{j}\right| .
$$

Furthermore, we claim that $\sum_{j=2}^{M}\left|a_{j}\right| \geq 1$ so that $g\left(\boldsymbol{v}_{\mathbf{0}}+\boldsymbol{w}\right) \geq C_{M}\left|\boldsymbol{v}_{\mathbf{0}}+\boldsymbol{w}\right|^{2}$. In fact, since by hypothesis $\{\boldsymbol{v}: g(\boldsymbol{v}) \leq 1\} \subseteq\{\boldsymbol{v}:|\boldsymbol{v}| \leq 1\}$, it follows that

$$
F(1)=g\left(\frac{\boldsymbol{v}_{\mathbf{0}}+\boldsymbol{w}}{\left|\boldsymbol{v}_{\mathbf{0}}+\boldsymbol{w}\right|}\right) \geq 1
$$

Therefore, $\sum_{j=2}^{M}\left|a_{j}\right| \geq \sum_{j=2}^{M} a_{j}=F(1) \geq 1$. Also, by equation (38), it follows that for $\lambda>\left(\frac{\sqrt{2}}{\sqrt{2}-1}\right)^{B}$,

$$
g\left(\boldsymbol{v}_{\mathbf{0}}+\boldsymbol{w}\right) \geq C_{M}\left|\boldsymbol{v}_{\mathbf{0}}+\boldsymbol{w}\right|^{2} \geq C_{M}|\boldsymbol{w}|^{2}\left(1-\frac{1}{\lambda^{\frac{1}{B}}}\right)^{2} \geq \frac{C_{M}|\boldsymbol{w}|^{2}}{2} .
$$

We would now like to obtain an upper bound for $\left|g\left(\boldsymbol{v}_{\mathbf{0}}\right)\right|$. Recall that by Claim 4.3, for any $\boldsymbol{v} \in \mathbb{R}^{n}$ we have that $g(\boldsymbol{v}) \leq C(1+r(\boldsymbol{v}))$. Thus, and since $\max \left\{2 m_{1}, \ldots, 2 m_{n}\right\}=B / 2<B$,

$$
\left|g\left(\boldsymbol{v}_{\mathbf{0}}\right)\right| \leq C\left(1+\left|\boldsymbol{v}_{\mathbf{0}}\right|^{2 m_{1}}+\ldots+\left|\boldsymbol{v}_{\mathbf{0}}\right|^{2 m_{n}}\right) \leq C\left(1+n+n\left|\boldsymbol{v}_{\mathbf{0}}\right|^{B}\right)<C\left(1+n+\frac{n|\boldsymbol{w}|}{\lambda}\right) .
$$

Thus, for $\lambda>\frac{8 C n}{C_{M}}$, it follows that

$$
\left|g\left(\boldsymbol{v}_{\mathbf{0}}\right)\right| \leq C\left(1+n+\frac{n}{\lambda}+\frac{n|\boldsymbol{w}|^{2}}{\lambda}\right) \leq C\left(1+n+\frac{n}{\lambda}\right)+\frac{C_{M}|\boldsymbol{w}|^{2}}{8} .
$$


It now suffices to obtain an upper bound for $\left|\nabla g\left(\boldsymbol{v}_{\mathbf{0}}\right) \cdot \boldsymbol{w}\right|$. Notice that for each $1 \leq j \leq n$, the $j^{\text {th }}$ entry of $\nabla g$ is a polynomial whose exponents satisfy

$$
\frac{2 \alpha_{1}}{B}+\cdots+\frac{2 \alpha_{n}}{B} \leq \frac{\alpha_{1}}{2 m_{1}}+\cdots+\frac{\alpha_{n}}{2 m_{n}} \leq 1-\frac{1}{2 m_{j}}<1
$$

That is, $\alpha_{1}+\ldots+\alpha_{n}<\frac{B}{2}$. Thus, we can bound each entry of $\left|\nabla g\left(\boldsymbol{v}_{\mathbf{0}}\right)\right|$ by a constant multiple of $1+\left|\boldsymbol{v}_{\mathbf{0}}\right|^{\frac{B}{2}}$. The coefficients of each of these entries are multiples of the coefficients of $g$, where the factors depend only on the degree of $g$. Thus, since $\sum_{\alpha \in \Gamma}\left|c_{\alpha}\right| \leq C$, there exists a constant $C_{1}$ that depends only on $C$ and the degree of $g$ such that

$$
\left|\nabla g\left(\boldsymbol{v}_{\mathbf{0}}\right)\right| \leq C_{1}\left(1+\left|\boldsymbol{v}_{\mathbf{0}}\right|^{\frac{B}{2}}\right) .
$$

Hence, in the region under consideration we have

$$
\left|\nabla g\left(\boldsymbol{v}_{\mathbf{0}}\right)\right| \leq C_{1}\left(1+\frac{|\boldsymbol{w}|^{\frac{1}{2}}}{\lambda^{\frac{1}{2}}}\right)
$$

It follows that

$$
\left|\nabla g\left(\boldsymbol{v}_{\mathbf{0}}\right) \cdot \boldsymbol{w}\right| \leq\left|\nabla g\left(\boldsymbol{v}_{\mathbf{0}}\right)\right||\boldsymbol{w}| \leq C_{1}\left(1+\frac{|\boldsymbol{w}|^{\frac{1}{2}}}{\lambda^{\frac{1}{2}}}\right)|\boldsymbol{w}|=C_{1}\left(|\boldsymbol{w}|+\frac{|\boldsymbol{w}|^{\frac{3}{2}}}{\lambda^{\frac{1}{2}}}\right) .
$$

Since $|\boldsymbol{w}| \leq \frac{1}{A}|\boldsymbol{w}|^{2}+A^{2}$ for any constant $A>0$, and since $|\boldsymbol{w}|^{\frac{3}{2}} \leq|\boldsymbol{w}|^{2}+1$, we have that

$$
\left|\nabla g\left(\boldsymbol{v}_{\mathbf{0}}\right) \cdot \boldsymbol{w}\right| \leq C_{1}\left(\frac{1}{A}|\boldsymbol{w}|^{2}+A^{2}+\frac{|\boldsymbol{w}|^{2}}{\lambda^{\frac{1}{2}}}+\frac{1}{\lambda^{\frac{1}{2}}}\right) .
$$

Then for $A>\frac{16 C_{1}}{C_{M}}$ and $\lambda>\left(\frac{16 C_{1}}{C_{M}}\right)^{2}$ it follows that

$$
\left|\nabla g\left(\boldsymbol{v}_{\mathbf{0}}\right) \cdot \boldsymbol{w}\right| \leq C_{1}\left(A^{2}+\frac{1}{\lambda^{\frac{1}{2}}}\right)+\frac{C_{M}|\boldsymbol{w}|^{2}}{8} .
$$

Therefore, by equations (39),(40) and (41), and taking

$$
\lambda>\max \left\{\left(\frac{\sqrt{2}}{\sqrt{2}-1}\right)^{B}, \frac{8 C n}{C_{M}},\left(\frac{16 C_{1}}{C_{M}}\right)^{2}\right\}
$$

it follows that

$$
f(\boldsymbol{w}) \geq|\boldsymbol{w}|^{2}\left(\frac{C_{M}}{2}-\frac{C_{M}}{8}-\frac{C_{M}}{8}\right)-E=\frac{C_{M}|\boldsymbol{w}|^{2}}{4}-E,
$$

where $E$ is a constant that depends on the combined degree of $g$ and the dimension of the space, but is otherwise independent.

Recall that our goal is to obtain an upper bound for

$$
I_{s}=\int_{\substack{|\boldsymbol{w}|>1 \\|\boldsymbol{w}|>\lambda\left|\boldsymbol{v}_{\mathbf{0}}\right|^{B}}}|\boldsymbol{w}|^{s} e^{-f(\boldsymbol{w})} d \boldsymbol{w} .
$$

Using the lower bound for $f(\boldsymbol{w})$ obtained in equation(42) we have that 


$$
I_{s} \lesssim \int_{\substack{|\boldsymbol{w}|>1 \\|\boldsymbol{w}|>\lambda\left|\boldsymbol{v}_{0}\right|^{B}}}|\boldsymbol{w}|^{s} e^{\frac{-C_{M}|\boldsymbol{w}|^{2}}{4}} d \boldsymbol{w} \lesssim \int_{0}^{\infty} r^{s} e^{\frac{-C_{M} r^{2}}{4}} d r
$$

Since $C_{M}$ is a strictly positive constant, the above integral converges. This finishes the proof of Claim 4.11.

It follows from equation (24) and Claim 4.11 that the derivatives of $\theta(\boldsymbol{\eta})$ are bounded from above by a sum of terms of the form

$$
\frac{H_{f}\left[\left|\boldsymbol{v}_{\mathbf{0}}\right|\right]^{d-1}}{e^{h\left(\boldsymbol{v}_{\mathbf{0}}\right)}|\{\boldsymbol{w}: f(\boldsymbol{w}) \leq 1\}|^{d}}
$$

Moreover, by Claim 4.11 these terms can be bounded by terms of the form

$$
\frac{q\left(\left|\boldsymbol{v}_{\mathbf{0}}\right|\right)}{e^{h\left(\boldsymbol{v}_{\mathbf{0}}\right)}|\{\boldsymbol{w}: f(\boldsymbol{w}) \leq 1\}|^{k}}
$$

where $q: \mathbb{R} \rightarrow \mathbb{R}$ is a polynomial, and $k \in[1, d] \cap \mathbb{Z}$. By Claim 4.8 it follows that $q\left(\left|\boldsymbol{v}_{\mathbf{0}}\right|\right)$ is bounded by a polynomial in $|\boldsymbol{\eta}|$. Furthermore, by Claim 4.7, $|\{\boldsymbol{w}: f(\boldsymbol{w}) \leq 1\}|^{-k} \lesssim\left(1+r\left(\boldsymbol{v}_{\mathbf{0}}\right)\right)^{\frac{k n}{2}}$. By Claim 4.9 this latter bound is at most of polynomial growth in $|\boldsymbol{\eta}|$. Thus, the derivatives of $\theta(\boldsymbol{\eta})$ are bounded by sums of terms of the form $e^{-h\left(\boldsymbol{v}_{\mathbf{0}}\right)} \tilde{q}(|\boldsymbol{\eta}|)$, where $\tilde{q}$ grows at a polynomial rate. Finally, by equation (21) $e^{-h\left(\boldsymbol{v}_{\mathbf{0}}\right)}$ decays at an exponential rate in $|\boldsymbol{\eta}|$. This finishes the proof that the derivatives of $\theta(\boldsymbol{\eta})$ decay exponentially. Thus, $\theta$ is a Schwartz function. Moreover, it follows from the previous computations that its decay is independent of the coefficients of $g$.

\section{BOUNDS FOR THE SZEGŐ KERNEL}

In this section we present the proof of our main result. With $\Omega_{b}$ defined as before, let $(\boldsymbol{x}, \boldsymbol{y}, t)$ and $\left(\boldsymbol{x}^{\prime}, \boldsymbol{y}^{\prime}, t^{\prime}\right)$ be any two points in $\partial \Omega_{b}$. Define

$$
\begin{gathered}
\tilde{b}(\boldsymbol{v})=b\left(\boldsymbol{v}+\frac{\boldsymbol{x}+\boldsymbol{x}^{\prime}}{2}\right)-\nabla b\left(\frac{\boldsymbol{x}+\boldsymbol{x}^{\prime}}{2}\right) \cdot \boldsymbol{v}-b\left(\frac{\boldsymbol{x}+\boldsymbol{x}^{\prime}}{2}\right) \\
\delta\left(\boldsymbol{x}, \boldsymbol{x}^{\prime}\right)=b(\boldsymbol{x})+b\left(\boldsymbol{x}^{\prime}\right)-2 b\left(\frac{\boldsymbol{x}+\boldsymbol{x}^{\prime}}{2}\right)
\end{gathered}
$$

and

$$
w=\left(t^{\prime}-t\right)+\nabla b\left(\frac{\boldsymbol{x}+\boldsymbol{x}^{\prime}}{2}\right) \cdot\left(\boldsymbol{y}^{\prime}-\boldsymbol{y}\right) .
$$

We obtain the following estimate for the Szegö kernel associated to the domain $\Omega_{b}$ :

$$
\left|S\left((\boldsymbol{x}, \boldsymbol{y}, t) ;\left(\boldsymbol{x}^{\prime}, \boldsymbol{y}^{\prime}, t^{\prime}\right)\right)\right| \leq \frac{C}{\sqrt{\delta^{2}+\widetilde{b}\left(\boldsymbol{y}-\boldsymbol{y}^{\prime}\right)^{2}+w^{2}}\left|\left\{\boldsymbol{v}: \tilde{b}(\boldsymbol{v})<\sqrt{\delta^{2}+\widetilde{b}\left(\boldsymbol{y}-\boldsymbol{y}^{\prime}\right)^{2}+w^{2}}\right\}\right|^{2}} .
$$

Here the constant $C$ depends on the exponents $\left\{m_{1}, \ldots, m_{n}\right\}$ and the dimension of the space, but is independent of the two given points. 
Remark 5.1. Here, $\tilde{b}$ is a strictly convex polynomial of the same combined degree as b, but with $\tilde{b}(\mathbf{0})=0$, and $\nabla \tilde{b}(\mathbf{0})=\mathbf{0}$. Notice that we can write $\tilde{b}(\boldsymbol{v})=f(\boldsymbol{v})-L(\boldsymbol{v})$, where $f(\boldsymbol{v})=b\left(\boldsymbol{v}+\frac{\boldsymbol{x}+\boldsymbol{x}^{\prime}}{2}\right)$ and $L$ is the tangent hyperplane to $f$ at $\boldsymbol{v}=0$.

Remark 5.2. Since $b$ is strictly convex, $\delta\left(\boldsymbol{x}, \boldsymbol{x}^{\prime}\right)>0$.

We obtain this bound by estimating the integral expression for the Szegö kernel obtained in Proposition 2.1. That is, we study

$$
S\left((\boldsymbol{x}, \boldsymbol{y}, t) ;\left(\boldsymbol{x}^{\prime}, \boldsymbol{y}^{\prime}, t^{\prime}\right)\right)=\int_{0}^{\infty} e^{-2 \pi \tau\left[b\left(\boldsymbol{x}^{\prime}\right)+b(\boldsymbol{x})+i\left(t^{\prime}-t\right)\right]}\left(\int_{\mathbb{R}^{n}} \frac{e^{2 \pi \boldsymbol{\eta} \cdot\left[\boldsymbol{x}+\boldsymbol{x}^{\prime}-i\left(\boldsymbol{y}^{\prime}-\boldsymbol{y}\right)\right]}}{\int_{\mathbb{R}^{n}} e^{4 \pi[\boldsymbol{\eta} \cdot \boldsymbol{v}-b(\boldsymbol{v}) \tau]} d \boldsymbol{v}} d \boldsymbol{\eta}\right) d \tau
$$

The proof is in essence an application of John ellipsoids. Recall that by John [17], given a symmetric convex compact region, there exists a maximal inscribed ellipsoid $\mathfrak{E}$ in that region (centered at the center of symmetry) such that $\sqrt{n} \mathfrak{E}$ contains the region, where $n$ is the dimension of the space and $\sqrt{n} \mathfrak{E}$ is the dilation of $\mathfrak{E}$ relative to its center of symmetry. The key step of our proof consists in introducing factors $\mu_{1}\left(\boldsymbol{x}, \boldsymbol{x}^{\prime}, \tau\right), \ldots, \mu_{n}\left(\boldsymbol{x}, \boldsymbol{x}^{\prime}, \tau\right)$ via a change of variable so that

$$
\mu_{1} \cdots \mu_{n} \approx\left|\left\{\boldsymbol{v}: \tilde{b}(\boldsymbol{v}) \leq \frac{1}{\tau}\right\}\right|
$$

These factors are chosen to be the length of the axes of the John ellipsoid associated to a symmetrization of the convex region $\left\{\boldsymbol{v}: \tilde{b}(\boldsymbol{v}) \leq \frac{1}{\tau}\right\}$. We explain this construction in the following subsection.

\subsection{Construction of the factors $\mu_{1} \ldots, \mu_{n}$. Let}

$$
R=\left\{\boldsymbol{v}: \tilde{b}(\boldsymbol{v}) \leq \frac{1}{\tau}\right\} .
$$

Notice that since $b$ is convex, so is $\tilde{b}$, and the region $\mathrm{R}$ is convex. In order to be able to use John's bounds, we need to show that the set $R$ is also compact. We do so in the following claim.

Claim 5.3. For any $M>0$, the set $\{\boldsymbol{v}: \tilde{b}(\boldsymbol{v}) \leq M\}$ is compact.

Proof. Notice that since $\tilde{b}$ is convex, $\tilde{b}(\mathbf{0})=0$, and $\nabla \tilde{b}(\mathbf{0})=\mathbf{0}$, then $\tilde{b} \geq 0$ (and $\tilde{b}$ is not the zero polynomial).

Suppose towards a contradiction that the set $\{\boldsymbol{v}: \tilde{b}(\boldsymbol{v}) \leq M\}$ is unbounded. Then, by compactness of the unit ball, there exists a $\boldsymbol{x} \in \mathbb{R}^{n}$ such that $\forall c>0, \tilde{b}(c \boldsymbol{x}) \leq M$. In particular, since $\tilde{b}(\mathbf{0})=0$ and $\tilde{b}$ is convex, $\forall c>0, \tilde{b}(c \boldsymbol{x}) \equiv 0$. For $\boldsymbol{x}=\left(x_{1}, \ldots, x_{n}\right)$ as above, define $\boldsymbol{w}(t)=t \cdot\left(x_{1}, \ldots, x_{n}\right)$. Then since $\tilde{b}(c \boldsymbol{x}) \equiv 0$ for all $c>0$, it follows that $\tilde{b}(\boldsymbol{w}(t)) \equiv 0$.

On the other hand, since $\tilde{b}$ is of combined degree, its highest order terms corresponds to pure terms. Thus, the highest degree terms of $\tilde{b}(\boldsymbol{w}(t))$ are of the form $c_{\alpha}\left(t x_{i}\right)^{2 m_{i}}$, for some $i$ 's. In other words, the highest degree terms have coefficients of the form $c_{\alpha} x_{i}^{2 m_{i}}$, for some $i$ 's. The coefficients $c_{\alpha}$ of the highest degree pure terms of $\tilde{b}$ are positive, since $\tilde{b}\left(0, \ldots, 0, t x_{i}, 0, \ldots, 0\right)$ takes positive values. Therefore, the sum of the coefficients $c_{\alpha} x_{i}^{2 m_{i}}$ of the highest degree terms of $\tilde{b} \circ \boldsymbol{w}$ are also 

positive. Thus, $\tilde{b} \circ \boldsymbol{w}$ is not the zero polynomial. This yields the desired contradiction. It follows that $\{\boldsymbol{v}: \tilde{b}(\boldsymbol{v}) \leq M\}$ is bounded, and therefore compact.

Recall that by construction $\tilde{b}(\mathbf{0})=0$, so the region $R$ contains the origin. We would now like to show that there exists an ellipsoid $\mathfrak{E}$ centered at the origin such that

$$
\mathfrak{E} \subseteq R \subseteq C \mathfrak{E}
$$

for some independent positive constant $C$. The existence of such an ellipsoid would follow immediately by John if the region were symmetric (with the origin as center of symmetry). However, we have made no symmetry assumptions on our domain. Nevertheless, we can show the following:

Claim 5.4. Let $L$ be any line through the origin. This line $L$ will intersect $R$ in two points. Let $d_{1}$ be the shortest distance along $L$ from the origin to the boundary of $R$, and let $d_{2}$ be the largest distance. Then there exist constants $m, M$ depending only on the degree of the polynomial $b$ such that

$$
0<m \leq \frac{d_{2}}{d_{1}} \leq M<+\infty
$$

Proof. Let $h(\boldsymbol{v})=\tau \tilde{b}(\boldsymbol{v})$. Along the line $L$, the polynomial $h(\boldsymbol{v})$ is a polynomial of one variable which we will call $h_{L}(t)$. This polynomial satisfies $h_{L}(0)=h_{L}^{\prime}(0)=0$. Write

$$
h_{L}(t)=\sum_{j=2}^{N} c_{j} t^{j} .
$$

Then there exists some $2 \leq k \leq N$, and $\left|c_{k}\right| \neq 0$, such that

$$
h_{L}\left(d_{1}\right) \leq \sum_{j=2}^{N}\left|c_{j}\right| d_{1}^{j} \leq(N-1)\left|c_{k}\right| d_{1}^{k} .
$$

On the other hand, it follows from Lemma 2.1 on [5] that there exists a constant $0<C_{N} \leq 1$ such that

$$
h_{L}\left(d_{2}\right) \geq C_{N} \sum_{j=2}^{N}\left|c_{j}\right| d_{2}^{j} .
$$

For $k$ as above, it follows that

$$
C_{N}\left|c_{k}\right| d_{2}^{k} \leq C_{N} \sum_{j=2}^{N}\left|c_{j}\right| d_{2}^{j} \leq h_{L}\left(d_{2}\right) .
$$

Moreover, $h_{L}\left(d_{1}\right)=h_{L}\left(d_{2}\right)=1$, since $d_{1}$ and $d_{2}$ where chosen as the distances where $L$ intersects the boundary of the region $R=\{\boldsymbol{v}: h(\boldsymbol{v}) \leq 1\}$. Thus,

$$
C_{N}\left|c_{k}\right| d_{2}^{k} \leq h_{L}\left(d_{2}\right)=1=h_{L}\left(d_{1}\right) \leq(N-1)\left|c_{k}\right| d_{1}^{k}
$$

Therefore, 


$$
\frac{d_{2}}{d_{1}} \leq\left(\frac{N-1}{C_{N}}\right)^{\frac{1}{k}}
$$

On the other hand, since $d_{1}$ is the shortest distance along $L$ from $R$ to the origin and $d_{2}$ is the largest, it follows that $d_{1} \leq d_{2}$. Choosing $M=\max _{2 \leq k \leq N}\left\{\left(\frac{N-1}{C_{N}}\right)^{\frac{1}{k}}\right\}$ and $m=1$ it follows that

This finishes the proof of Claim 5.4.

$$
m \leq \frac{d_{2}}{d_{1}} \leq M
$$

We have shown that even though the region $R$ is not symmetric, the ratio between rays passing through the origin is bounded by universal constants that only depend on the degree of $b$. In the following lemma we will show that this is enough to guarantee the existence of an ellipsoid centered at the origin contained in $R$ and such that a dilation by a universal constant contains $R$.

Lemma 5.5. Let $R=\left\{\boldsymbol{v}: \tilde{b}(\boldsymbol{v}) \leq \frac{1}{\tau}\right\}$ and $\widetilde{R}=\{\boldsymbol{x}:-\boldsymbol{x} \in R\}$. Let $\mathfrak{E}$ be the maximal inscribed ellipsoid in the region $R \cap \widetilde{R}$. Then

$$
\mathfrak{E} \subseteq R \subseteq M \sqrt{n} \mathfrak{E}
$$

where $M$ is as in Claim 5.4, and $n$ is the dimension of the space.

Proof. By definition, the set $R \cap \widetilde{R}$ is symmetric about the origin. Moreover, since $R$ and $\widetilde{R}$ are compact and convex, their intersection is also compact and convex. It follows from John that there exists an ellipsoid $\mathfrak{E}$ centered at the origin such that

$$
\mathfrak{E} \subseteq R \cap \widetilde{R} \subseteq \sqrt{n} \mathfrak{E}
$$

It is clear that $\mathfrak{E} \subseteq R$. We would like to show that there is a dilation of $\mathfrak{E}$ which contains $R$. Let $\boldsymbol{x}$ be any point in $R$. Then, if $-\boldsymbol{x} \in R$, it follows by definition that $x \in \sqrt{n} \mathfrak{E}$. Now suppose that $-\boldsymbol{x} \notin R$. Let $L$ be the line that goes through the origin and $\boldsymbol{x}$. Using the notation of the previous claim, we have that $|\boldsymbol{x}| \leq d_{2}$. Given $M$ as in Claim 5.4, let $\rho=\frac{1}{M}$. Then $|-\rho \boldsymbol{x}| \leq \rho d_{2} \leq \rho M d_{1}=d_{1}$. But since $d_{1}$ is the minimum distance from the boundary of $R$ to the origin along line $L$, it follows that $-\rho \boldsymbol{x} \in R$. Thus, given any point $\boldsymbol{x} \in R$ the point $-\frac{1}{M} \boldsymbol{x}$ is also contained in $R$. It follows that

$$
\mathfrak{E} \subseteq R \subseteq M \sqrt{n} \mathfrak{E}
$$

This finishes the proof of Lemma 5.5.

It follows from the previous lemma that

$$
\operatorname{Vol}(\mathfrak{E}) \approx\left|\left\{\boldsymbol{v}: \tilde{b}(\boldsymbol{v}) \leq \frac{1}{\tau}\right\}\right|
$$

Let $\mu_{1}, \ldots, \mu_{n}$ be the lengths of the semi-axes of $\sqrt{n} M \mathfrak{E}$, indexed so that $\mu_{1} \geq \mu_{2} \geq \ldots \geq \mu_{n}$. Then, 


$$
\mu_{1} \cdots \mu_{n} \approx\left|\left\{\boldsymbol{v}: \tilde{b}(\boldsymbol{v}) \leq \frac{1}{\tau}\right\}\right|
$$

In equations (46) and (47), the constant depends only on the combined degree of $b$ and the dimension of the space.

5.2. Proof of the Main Theorem. We are now ready to present the proof of the Main Theorem. For the reader's convenience, we have divided the proof into three subsections, corresponding to a bound in terms of $\delta$, a bound in terms of $\widetilde{b}\left(\boldsymbol{y}-\boldsymbol{y}^{\prime}\right)$ and a bound in terms of $w$. We finish by combining all three bounds to obtain the estimate stated in the Main Theorem. It will be convenient in the course of the proof of all three bounds to rearrange the terms of the integral expression for the Szegö kernel obtained in Theorem 2.1 as follows:

Making the change of variables $\boldsymbol{v} \rightarrow \boldsymbol{v}+\frac{\boldsymbol{x}}{2}+\frac{\boldsymbol{x}^{\prime}}{2}$ to get rid of the term $e^{2 \pi \boldsymbol{\eta} \cdot\left(\boldsymbol{x}+\boldsymbol{x}^{\prime}\right)}$ in the original expression obtained for the Szegő kernel given by

$$
S\left((\boldsymbol{x}, \boldsymbol{y}, t) ;\left(\boldsymbol{x}^{\prime}, \boldsymbol{y}^{\prime}, t^{\prime}\right)\right)=\int_{0}^{\infty} e^{-2 \pi \tau\left[b\left(\boldsymbol{x}^{\prime}\right)+b(\boldsymbol{x})+i\left(t^{\prime}-t\right)\right]}\left(\int_{\mathbb{R}^{n}} \frac{e^{2 \pi \boldsymbol{\eta} \cdot\left[\boldsymbol{x}+\boldsymbol{x}^{\prime}-i\left(\boldsymbol{y}^{\prime}-\boldsymbol{y}\right)\right]}}{\int_{\mathbb{R}^{n}} e^{4 \pi[\boldsymbol{\eta} \cdot \boldsymbol{v}-b(\boldsymbol{v}) \tau]} d \boldsymbol{v}} d \boldsymbol{\eta}\right) d \tau
$$

it follows that

$$
S=\int_{0}^{\infty} e^{-2 \pi \tau\left[b\left(\boldsymbol{x}^{\prime}\right)+b(\boldsymbol{x})+i\left(t^{\prime}-t\right)\right]} \int_{\mathbb{R}^{n}} \frac{e^{2 \pi i \boldsymbol{\eta} \cdot\left(\boldsymbol{y}-\boldsymbol{y}^{\prime}\right)}}{\int_{\mathbb{R}^{n}} e^{4 \pi\left[\boldsymbol{\eta} \cdot \boldsymbol{v}-\tau b\left(\boldsymbol{v}+\frac{\boldsymbol{x}+\boldsymbol{x}^{\prime}}{2}\right)\right]} d \boldsymbol{v}} d \boldsymbol{\eta} d \tau
$$

We will modify the denominator integral so as to change it into an integral of the form $[\theta(\boldsymbol{\eta})]^{-1}$, where $\theta$ is the function studied in Lemma 4.1. In particular, the exponent of the denominator integral must be of the form $\boldsymbol{\eta} \cdot \boldsymbol{v}-g(\boldsymbol{v})$, where $g(\mathbf{0})=0$ and $\nabla g(\mathbf{0})=0$. We make the change of variables $\boldsymbol{\eta} \rightarrow \boldsymbol{\eta}+\nabla b\left(\frac{\boldsymbol{x}+\boldsymbol{x}^{\prime}}{2}\right) \tau$ so that

$$
S=\int_{0}^{\infty} e^{-2 \pi \tau\left[b\left(\boldsymbol{x}^{\prime}\right)+b(\boldsymbol{x})+i\left(t^{\prime}-t\right)\right]} e^{4 \pi \tau b\left(\frac{\boldsymbol{x}+\boldsymbol{x}^{\prime}}{2}\right)} e^{2 \pi i \tau \nabla b\left(\frac{\boldsymbol{x}+\boldsymbol{x}^{\prime}}{2}\right) \cdot\left(\boldsymbol{y}-\boldsymbol{y}^{\prime}\right)} \int_{\mathbb{R}^{n}} \frac{e^{2 \pi i \boldsymbol{\eta} \cdot\left(\boldsymbol{y}-\boldsymbol{y}^{\prime}\right)}}{\int_{\mathbb{R}^{n}} e^{4 \pi[\boldsymbol{\eta} \cdot \boldsymbol{v}-\tau \tilde{b}(\boldsymbol{v})]} d \boldsymbol{v}} d \boldsymbol{\eta} d \tau,
$$

where $\tilde{b}(\boldsymbol{v})$ is as in equation (43) and the term $b\left(\frac{\boldsymbol{x}+\boldsymbol{x}^{\prime}}{2}\right)$ has been added so that $\tilde{b}(\mathbf{0})=0$. With $\delta\left(\boldsymbol{x}, \boldsymbol{x}^{\prime}\right)$ and $w$ be as in equations (44) and (45), it follows that

$$
S=\int_{0}^{\infty} e^{-2 \pi \tau \delta} e^{-2 \pi \tau i w} \int_{\mathbb{R}^{n}} \frac{e^{2 \pi i \boldsymbol{\eta} \cdot\left(\boldsymbol{y}-\boldsymbol{y}^{\prime}\right)}}{\int_{\mathbb{R}^{n}} e^{4 \pi[\boldsymbol{\eta} \cdot \boldsymbol{v}-\tau \tilde{b}(\boldsymbol{v})]} d \boldsymbol{v}} d \boldsymbol{\eta} d \tau
$$

Notice that since $b$ is strictly convex, $\tau \tilde{b}$ is also strictly convex. Moreover, if $b$ is of combined degree $\left(m_{1}, \ldots, m_{n}\right)$, so is $\tau \tilde{b}$. Thus, $\tau \tilde{b}$ is a strictly convex polynomial satisfying conditions (i), (ii) and (iv) of Lemma 4.1. It remains to renormalize $\tau \tilde{b}$ so that it also satisfies condition (iii).

With $\mu_{1}, \ldots, \mu_{n}$ chosen as in equation (47), let

$$
g(\boldsymbol{v})=\tau \tilde{b}(\boldsymbol{\mu} \boldsymbol{v})
$$


Here, $\boldsymbol{\mu} \boldsymbol{v}=\left[\begin{array}{llll}\mu_{1} v_{1} & \mu_{2} v_{2} & \ldots & \mu_{n} v_{n}\end{array}\right]^{T}$. Notice that since $\mathfrak{E} \subseteq\{\boldsymbol{v}: \tau \tilde{b}(\boldsymbol{v}) \leq 1\} \subseteq \sqrt{n} M \mathfrak{E}$ and letting $A=(\sqrt{n} M)^{-1}$ condition (iii) of Lemma 4.1 is satisfied.

By making the change of variables $\boldsymbol{v} \rightarrow \boldsymbol{\mu} \boldsymbol{v}$ so as to introduce the factors $\mu_{1}, \ldots, \mu_{n}$ in the denominator integral of equation (48), as well as the change of variables $\boldsymbol{\eta} \rightarrow \frac{\boldsymbol{\eta}}{\boldsymbol{\mu}}$ we have that

$$
S=\int_{0}^{\infty} \frac{e^{-2 \pi \tau \delta} e^{-2 \pi \tau i w}}{\mu_{1}^{2} \cdots \mu_{n}^{2}} \int_{\mathbb{R}^{n}} \frac{e^{2 \pi i \boldsymbol{\eta} \cdot\left(\frac{\boldsymbol{y}-\boldsymbol{y}^{\prime}}{\boldsymbol{\mu}}\right)}}{\int_{\mathbb{R}^{n}} e^{4 \pi[\boldsymbol{\eta} \cdot \boldsymbol{v}-\tau \tilde{b}(\boldsymbol{\mu} \boldsymbol{v})]} d \boldsymbol{v}} d \boldsymbol{\eta} d \tau
$$

\subsubsection{The bound in terms of $\delta$.}

Proposition 5.6. Let $(\boldsymbol{x}, \boldsymbol{y}, t)$ and $\left(\boldsymbol{x}^{\prime}, \boldsymbol{y}^{\prime}, t^{\prime}\right)$ be any two points in $\partial \Omega_{b}$. Then,

$$
\left|S\left((\boldsymbol{x}, \boldsymbol{y}, t) ;\left(\boldsymbol{x}^{\prime}, \boldsymbol{y}^{\prime}, t^{\prime}\right)\right)\right| \lesssim \frac{1}{\delta|\{\boldsymbol{v}: \tilde{b}(\boldsymbol{v})<\delta\}|^{2}},
$$

where the constant may depend on the combined degree of $b$ and the dimension of the space, but is independent of the two given points.

Proof. It follows from equation (50) that

$$
|S| \leq \int_{0}^{\infty} \frac{e^{-2 \pi \tau \delta}}{\mu_{1}^{2} \cdots \mu_{n}^{2}} \int_{\mathbb{R}^{n}} \frac{1}{\int_{\mathbb{R}^{n}} e^{4 \pi[\boldsymbol{\eta} \cdot \boldsymbol{v}-\tau \tilde{b}(\mu \boldsymbol{v})]} d \boldsymbol{v}} d \boldsymbol{\eta} d \tau .
$$

But since $\mu_{1} \cdots \mu_{n} \approx\left|\left\{\boldsymbol{v}: \tilde{b}(\boldsymbol{v}) \leq \frac{1}{\tau}\right\}\right|$, it follows that

$$
|S| \lesssim \int_{0}^{\infty} \frac{e^{-2 \pi \tau \delta}}{\left|\left\{\boldsymbol{v}: \tilde{b}(\boldsymbol{v}) \leq \frac{1}{\tau}\right\}\right|^{2}} \int_{\mathbb{R}^{n}} \frac{1}{\int_{\mathbb{R}^{n}} e^{4 \pi[\boldsymbol{\eta} \cdot \boldsymbol{v}-\tau \tilde{b}(\mu \boldsymbol{v})]} d \boldsymbol{v}} d \boldsymbol{\eta} d \tau
$$

By Lemma 4.1 the reciprocal of the denominator integral is Schwartz, and the decay is independent of the coefficients of $g$. In particular, the decay does not depend on $\tau$, and $\int_{\mathbb{R}^{n}} \theta(\boldsymbol{\eta}) d \boldsymbol{\eta}$ converges. Hence,

We can write

$$
|S| \lesssim \int_{0}^{\infty} \frac{e^{-2 \pi \tau \delta}}{\left|\left\{\boldsymbol{v}: \tilde{b}(\boldsymbol{v}) \leq \frac{1}{\tau}\right\}\right|^{2}} d \tau
$$

Thus,

$$
\int_{0}^{\infty} \frac{e^{-2 \pi \tau \delta}}{\left|\left\{\boldsymbol{v}: \tilde{b}(\boldsymbol{v}) \leq \frac{1}{\tau}\right\}\right|^{2}} d \tau=\sum_{j=-\infty}^{\infty} \int_{\tau \delta=2^{j}}^{\tau \delta=2^{j+1}} \frac{e^{-2 \pi \tau \delta}}{\left|\left\{\boldsymbol{v}: \tilde{b}(\boldsymbol{v}) \leq \frac{1}{\tau}\right\}\right|^{2}} d \tau
$$

$$
|S| \lesssim \sum_{j=-\infty}^{\infty} \frac{e^{-2 \pi 2^{j}}}{\left|\left\{\boldsymbol{v}: \tilde{b}(\boldsymbol{v}) \leq \frac{\delta}{2^{j+1}}\right\}\right|^{2}} \int_{\tau=2^{j} \delta^{-1}}^{\tau=2^{j+1} \delta^{-1}} d \tau=\sum_{j=-\infty}^{\infty} \frac{2^{j} e^{-2 \pi 2^{j}}}{\delta\left|\left\{\boldsymbol{v}: \tilde{b}(\boldsymbol{v}) \leq \frac{\delta}{2^{j+1}}\right\}\right|^{2}}
$$


In order to get rid of the dependence on $j$ of $\left|\left\{\boldsymbol{v}: \tilde{b}(\boldsymbol{v}) \leq \frac{\delta}{2^{j+1}}\right\}\right|$ we can write

$$
|S| \lesssim \sum_{-\infty<j<0} \frac{e^{-2 \pi 2^{j}} 2^{j}}{\delta|\{\boldsymbol{v}: \tilde{b}(\boldsymbol{v}) \leq \delta\}|^{2}}+\sum_{0 \leq j<\infty} \frac{e^{-2 \pi 2^{j}} 2^{j}}{\delta\left|\left\{\boldsymbol{v}: \tilde{b}(\boldsymbol{v}) \leq \frac{\delta}{2^{j+1}}\right\}\right|^{2}}
$$

But for $j \geq 0$ we have that (see Claim 6.1 in the Appendix)

It follows that

$$
|\{\boldsymbol{v}: \tilde{b}(\boldsymbol{v}) \leq \delta\}| \leq 2^{n(j+1)}\left|\left\{\boldsymbol{v}: \tilde{b}(\boldsymbol{v}) \leq \frac{\delta}{2^{j+1}}\right\}\right|
$$

$$
|S| \lesssim \frac{1}{\delta|\{\boldsymbol{v}: \tilde{b}(\boldsymbol{v}) \leq \delta\}|^{2}}\left(\sum_{-\infty<j<0} e^{-2^{j+1} \pi} 2^{j}+\sum_{0 \leq j<\infty} e^{-2^{j+1} \pi} 2^{2 n(j+1)+j}\right) .
$$

Since both sums converge, we obtain the desired estimate.

5.2.2. The bound in terms of $\widetilde{b}\left(\boldsymbol{y}-\boldsymbol{y}^{\prime}\right)$.

Proposition 5.7. Let $(\boldsymbol{x}, \boldsymbol{y}, t)$ and $\left(\boldsymbol{x}^{\prime}, \boldsymbol{y}^{\prime}, t^{\prime}\right)$ be any two points in $\partial \Omega_{b}$. Then

$$
\left|S\left((\boldsymbol{x}, \boldsymbol{y}, t) ;\left(\boldsymbol{x}^{\prime}, \boldsymbol{y}^{\prime}, t^{\prime}\right)\right)\right| \lesssim \frac{1}{\widetilde{b}\left(\boldsymbol{y}-\boldsymbol{y}^{\prime}\right)\left|\left\{\boldsymbol{v}: \tilde{b}(\boldsymbol{v})<\widetilde{b}\left(\boldsymbol{y}-\boldsymbol{y}^{\prime}\right)\right\}\right|^{2}},
$$

where the constant may depend on the combined degree of $b$ and the dimension of the space, but is independent of the two given points.

Proof. We had shown in equation (50) on page 28 that

$$
S=\int_{0}^{\infty} \frac{e^{-2 \pi \tau \delta} e^{-2 \pi \tau i w}}{\mu_{1}^{2} \cdots \mu_{n}^{2}} \int_{\mathbb{R}^{n}} \frac{e^{2 \pi i \boldsymbol{\eta} \cdot\left(\frac{\boldsymbol{y}-\boldsymbol{y}^{\prime}}{\boldsymbol{\mu}}\right)}}{\int_{\mathbb{R}^{n}} e^{4 \pi[\boldsymbol{\eta} \cdot \boldsymbol{v}-\tau \tilde{b}(\boldsymbol{\mu} \boldsymbol{v})]} d \boldsymbol{v}} d \boldsymbol{\eta} d \tau .
$$

Thus, and since $\delta>0$,

$$
|S| \leq \int_{0}^{\infty} \frac{1}{\mu_{1}^{2} \cdots \mu_{n}^{2}}\left|\int_{\mathbb{R}^{n}} \frac{e^{2 \pi i \boldsymbol{\eta} \cdot\left(\frac{\boldsymbol{y}-\boldsymbol{y}^{\prime}}{\boldsymbol{\mu}}\right)}}{\int_{\mathbb{R}^{n}} e^{4 \pi[\boldsymbol{\eta} \cdot \boldsymbol{v}-\tau \tilde{b}(\boldsymbol{\mu} \boldsymbol{v})]} d \boldsymbol{v}} d \boldsymbol{\eta}\right| d \tau .
$$

But by Lemma 4.1, the reciprocal of the denominator integral is Schwartz. Moreover, its decay is independent of $\tau$ and the coefficients of $b$. The same is true of its Fourier transform, $\hat{\theta}$. We can write

$$
|S| \leq \int_{0}^{\infty} \frac{1}{\mu_{1}^{2} \cdots \mu_{n}^{2}}\left|\hat{\theta}\left(\frac{\boldsymbol{y}-\boldsymbol{y}^{\prime}}{\boldsymbol{\mu}}\right)\right| d \tau
$$

And as in the previous bound, we can write

$$
|S| \lesssim \int_{0}^{\infty} \frac{1}{\left|\left\{\boldsymbol{v}: \widetilde{b}(\boldsymbol{v}) \leq \frac{1}{\tau}\right\}\right|^{2}}\left|\hat{\theta}\left(\frac{\boldsymbol{y}-\boldsymbol{y}^{\prime}}{\boldsymbol{\mu}}\right)\right| d \tau
$$

Let $\boldsymbol{\gamma}=\boldsymbol{y}-\boldsymbol{y}^{\prime}$. We can split the interval of integration into dyadic intervals in the following way: 


$$
\int_{0}^{\infty} \frac{1}{\left|\left\{\boldsymbol{v}: \widetilde{b}(\boldsymbol{v}) \leq \frac{1}{\tau}\right\}\right|^{2}}\left|\hat{\theta}\left(\frac{\boldsymbol{y}-\boldsymbol{y}^{\prime}}{\boldsymbol{\mu}}\right)\right| d \tau=\sum_{j=-\infty}^{\infty} \int_{2^{j}(\widetilde{b}(\gamma))^{-1}}^{2^{j+1}(\widetilde{b}(\gamma))^{-1}} \frac{1}{\left|\left\{\boldsymbol{v}: \widetilde{b}(\boldsymbol{v}) \leq \frac{1}{\tau}\right\}\right|^{2}}\left|\hat{\theta}\left(\frac{\boldsymbol{\gamma}}{\boldsymbol{\mu}}\right)\right| d \tau
$$

But since in each interval $\frac{\widetilde{b}(\gamma)}{2^{j+1}} \leq \frac{1}{\tau}$, It follows that

$$
\begin{aligned}
|S| \lesssim & \sum_{-\infty<j<0} \int_{2^{j}(\widetilde{b}(\gamma))^{-1}}^{\left.2^{j+1} \widetilde{b}(\gamma)\right)^{-1}} \frac{1}{\left|\left\{\boldsymbol{v}: \widetilde{b}(\boldsymbol{v}) \leq \frac{\widetilde{b}(\gamma)}{2^{j+1}}\right\}\right|^{2}}\left|\hat{\theta}\left(\frac{\gamma}{\boldsymbol{\mu}}\right)\right| d \tau \\
& +\sum_{0 \leq j<\infty} \int_{2^{j}(\widetilde{b}(\gamma))^{-1}}^{2^{j+1}(\widetilde{b}(\gamma))^{-1}} \frac{1}{\left|\left\{\boldsymbol{v}: \widetilde{b}(\boldsymbol{v}) \leq \frac{\widetilde{b}(\gamma)}{2^{j+1}}\right\}\right|^{2}}\left|\hat{\theta}\left(\frac{\gamma}{\boldsymbol{\mu}}\right)\right| d \tau
\end{aligned}
$$

As in the previous bound, for $j<0$ we have that $\left|\left\{\boldsymbol{v}: \widetilde{b}(\boldsymbol{v}) \leq \frac{\widetilde{b}(\gamma)}{2^{j+1}}\right\}\right|^{-1} \leq|\{\boldsymbol{v}: \widetilde{b}(\boldsymbol{v}) \leq \widetilde{b}(\gamma)\}|^{-1}$, and for $j \geq 0$,

$$
\left|\left\{\boldsymbol{v}: \widetilde{b}(\boldsymbol{v}) \leq \frac{\widetilde{b}(\gamma)}{2^{j+1}}\right\}\right|^{-1} \leq 2^{n(j+1)}|\{\boldsymbol{v}: \widetilde{b}(\boldsymbol{v}) \leq \widetilde{b}(\boldsymbol{\gamma})\}|^{-1}
$$

Hence,

$$
\begin{aligned}
|S| & \lesssim \sum_{-\infty<j<0} \frac{1}{|\{\boldsymbol{v}: \widetilde{b}(\boldsymbol{v}) \leq \widetilde{b}(\gamma)\}|^{2}} \int_{2^{j}(\widetilde{b}(\gamma))^{-1}}^{2^{j+1}(\widetilde{b}(\gamma))^{-1}}\left|\hat{\theta}\left(\frac{\gamma}{\boldsymbol{\mu}}\right)\right| d \tau \\
& +\sum_{0 \leq j<\infty} \frac{2^{2 n(j+1)}}{|\{\boldsymbol{v}: \widetilde{b}(\boldsymbol{v}) \leq \widetilde{b}(\gamma)\}|^{2}} \int_{2^{j}(\widetilde{b}(\gamma))^{-1}}^{2^{j+1}(\widetilde{b}(\gamma))^{-1}}\left|\hat{\theta}\left(\frac{\gamma}{\boldsymbol{\mu}}\right)\right| d \tau
\end{aligned}
$$

For the first sum it suffices to bound $|\hat{\theta}|$ by a universal constant. It follows that

$$
\begin{aligned}
& \sum_{-\infty<j<0} \frac{1}{|\{\boldsymbol{v}: \widetilde{b}(\boldsymbol{v}) \leq \widetilde{b}(\gamma)\}|^{2}} \int_{2^{j}(\widetilde{b}(\gamma))^{-1}}^{2^{j+1}(\widetilde{b}(\gamma))^{-1}}\left|\hat{\theta}\left(\frac{\gamma}{\boldsymbol{\mu}}\right)\right| d \tau \\
\lesssim & \sum_{-\infty \leq j<0} \frac{1}{|\{\boldsymbol{v}: \widetilde{b}(\boldsymbol{v}) \leq \widetilde{b}(\gamma)\}|^{2}} \int_{2^{j}(\widetilde{b}(\gamma))^{-1}}^{2^{j+1}(\widetilde{b}(\gamma))^{-1}} d \tau \\
= & \sum_{-\infty \leq j<0} \frac{2^{j}}{\widetilde{b}(\gamma)|\{\boldsymbol{v}: \widetilde{b}(\boldsymbol{v}) \leq \widetilde{b}(\gamma)\}|^{2}} \approx \frac{1}{\widetilde{b}(\gamma)|\{\boldsymbol{v}: \widetilde{b}(\boldsymbol{v}) \leq \widetilde{b}(\gamma)\}|^{2}} .
\end{aligned}
$$

We would like to obtain a similar bound for the second sum, with $j \geq 0$. The main obstacle is obtaining decay in $j$ to counteract the growth of the term $2^{2 n(j+1)}$, thus ensuring the convergence of the series. We will show that 


$$
\int_{2^{j}(\widetilde{b}(\gamma))^{-1}}^{2^{j+1}(\widetilde{b}(\gamma))^{-1}}\left|\hat{\theta}\left(\frac{\gamma}{\boldsymbol{\mu}}\right)\right| d \tau \leq \frac{C_{k}}{2^{K j} \widetilde{b}(\gamma)}
$$

for any positive constant $K$, and a positive constant $C_{k}$ which depends only on $K$, on the combined degree of $b$, and on the dimension of the space.

Since $\hat{\theta}$ is Schwartz,

$$
\left|\hat{\theta}\left(\frac{\gamma}{\boldsymbol{\mu}}\right)\right| \leq \frac{D}{|C+| p\left(\frac{\gamma}{\mu}\right)||^{N}}
$$

for any polynomial $p: \mathbb{R}^{n} \rightarrow \mathbb{R}$ and positive constants $C$ and $N$. Here, the constant $D>0$ depends on $C, N$, and $p$. In this interval, $2^{j} \leq \tau \widetilde{b}(\gamma)$ so it suffices to show that there exists some polynomial $p$ and constant $C$ such that $\tau \widetilde{b}(\gamma) \leq C+\left|p\left(\frac{\gamma}{\mu}\right)\right|$. In fact, it would follow that for any $N>0$,

so that

$$
\left|\hat{\theta}\left(\frac{\gamma}{\boldsymbol{\mu}}\right)\right| \leq \frac{D}{|\tau \widetilde{b}(\gamma)|^{N}} \leq \frac{D}{2^{N j}}
$$

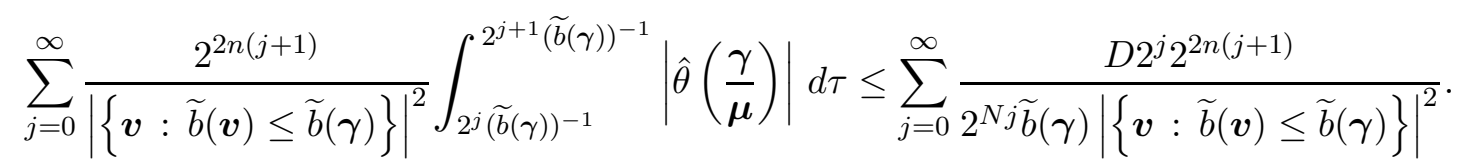

For sufficiently large $N$, the series converges, and we would obtain the desired estimate.

In order to find a polynomial $p$ and positive constant $C$ such that $\tau \widetilde{b}(\gamma) \leq C+\left|p\left(\frac{\gamma}{\mu}\right)\right|$, let $s=\frac{\gamma}{\mu}$ and write the above requirement as $\tau \widetilde{b}(\boldsymbol{\mu s}) \leq C+|p(\boldsymbol{s})|$. By construction of the factors $\mu_{1}, \ldots, \mu_{n}$ the polynomial $\tau \widetilde{b}(\boldsymbol{\mu} \boldsymbol{s})$ satisfies all the hypothesis of Lemma 4.1. In particular, Claim 4.3 on page 12 holds. Thus, there exists a constant $C$, which depends only on the combined degree of $b$ and on the dimension of the space, such that

$$
\tau \widetilde{b}(\boldsymbol{\mu s}) \leq C\left(1+s_{1}^{2 m_{1}}+\ldots+s_{n}^{2 m_{n}}\right) .
$$

This finishes the proof of Proposition 5.7.

\subsubsection{The bound in terms of $w$.}

Proposition 5.8. Let $(\boldsymbol{x}, \boldsymbol{y}, t)$ and $\left(\boldsymbol{x}^{\prime}, \boldsymbol{y}^{\prime}, t^{\prime}\right)$ be any two points in $\partial \Omega_{b}$. Then,

$$
\left|S\left((\boldsymbol{x}, \boldsymbol{y}, t) ;\left(\boldsymbol{x}^{\prime}, \boldsymbol{y}^{\prime}, t^{\prime}\right)\right)\right| \lesssim \frac{1}{|w||\{\boldsymbol{v}: \widetilde{b}(\boldsymbol{v})<|w|\}|^{2}}
$$

where the constant may depend on the combined degree of $b$ and the dimension of the space, but is independent of the two given points.

The derivation of this last bound is rather long and technical. Before giving all the technical details, however, we shall begin by briefly outlining the main ideas behind the proof. It follows from equation (48) on page 27 that 


$$
S=\int_{0}^{\frac{\pi}{|u|}} e^{-i u \tau} F(\tau) d \tau+\int_{\frac{\pi}{|u|}}^{\infty} e^{-i u \tau} F(\tau) d \tau
$$

where for convenience we have set $u=2 \pi w$, and

$$
F(\tau)=e^{-2 \pi \tau \delta} \int_{\mathbb{R}^{n}} \frac{e^{2 \pi i \boldsymbol{\eta} \cdot\left(\boldsymbol{y}-\boldsymbol{y}^{\prime}\right)}}{\int_{\mathbb{R}^{n}} e^{4 \pi[\boldsymbol{\eta} \cdot \boldsymbol{v}-\tau \tilde{b}(\boldsymbol{v})]} d \boldsymbol{v}} d \boldsymbol{\eta} .
$$

The integral for $0 \leq \tau \leq \frac{\pi}{u}$ in equation (53) yields the desired estimate by using similar techniques as those detailed in the proof of the previous two bounds. Thus, the main difficulty lies in estimating the integral for $\frac{\pi}{|u|} \leq \tau<\infty$. In particular, we must show that the integral converges. To do so, we will take advantage of the oscillation of the term $e^{-i u \tau}$. Integrating the latter by parts $N$ times, for an arbitrary positive integer $N$, we obtain formally an equation of the form

$$
\int_{\frac{\pi}{|u|}}^{\infty} \frac{e^{-i u \tau}}{|u|^{N}} F^{(N)}(\tau) d \tau
$$

We then show that after introducing the factors $\boldsymbol{\mu}$ as in the two previous bounds, every derivative of $F(\tau)$ yields a factor of $\frac{1}{\tau}$ times a bounded function, so that

$$
\left|\int_{\frac{\pi}{|u|}}^{\infty} \frac{e^{-i u \tau}}{|u|^{N}} F^{(N)}(\tau) d \tau\right| \approx \frac{1}{|u|^{N}} \int_{\frac{\pi}{|u|}}^{\infty} \frac{1}{\mu_{1}^{2} \cdots \mu_{n}^{2}} \cdot \frac{1}{\tau^{N}} d \tau \approx \frac{1}{|u|^{N}} \int_{\frac{\pi}{|u|}}^{\infty} \frac{1}{\left|\left\{\boldsymbol{v}: \tilde{b}(\boldsymbol{v}) \leq \frac{1}{\tau}\right\}\right|^{2}} \cdot \frac{1}{\tau^{N}} d \tau .
$$

Finally, using Claim 6.1 (see Appendix), we show that this last integral is bounded by an expression of the form

$$
\frac{1}{|u|^{N}|\{\boldsymbol{v}: \widetilde{b}(\boldsymbol{v}) \leq|u|\}|^{2}} \int_{\frac{\pi}{|u|}}^{\infty} \frac{|u|^{2 n} \tau^{2 n}}{\tau^{N}} d \tau,
$$

yielding the desired estimate for large enough values of $N$.

Before presenting a rigorous proof of Proposition 5.8, we discuss three technical results that will be used in the course of the proof. In Claim 5.9 we obtain an upper bound for $\int_{0}^{\infty} e^{-i u \tau} F(\tau) d \tau$ in terms of the $(N+1)^{s t}$ derivative of $F$. The method we use is analogous to integration by parts, but does not yield boundary terms, making the computation slightly simpler (see, e.g., Proposition $X_{19}$ on p. 14 [31]). In Claim 5.10 we compute the $N^{\text {th }}$ derivative of $F$. In Claim 5.11 we show that, after introducing the factors $\boldsymbol{\mu}$, the $N^{t h}$ derivative of $F$ is dominated by $\frac{1}{\tau^{N}}$ times a bounded function.

Claim 5.9. Let $t \in \mathbb{R}$ and

$$
I(t)=\int_{0}^{\infty} e^{-i t \tau} F(\tau) d \tau
$$

where $F \in C^{\infty}(\mathbb{R})$ and $F \in L^{1}(\mathbb{R})$. Then given $N \in \mathbb{N}$ there exist positive coefficients $c_{1}, \ldots, c_{N+1}$, such that 


$$
\begin{aligned}
|I(t)| \leq & \sum_{j=0}^{N+1} c_{j}\left|\int_{0}^{\frac{\pi}{|t|}} e^{-i t \tau} F\left(\tau+\frac{j \pi}{|t|}\right) d \tau\right| \\
& +\frac{1}{2^{N+1}}\left|\int_{\frac{\pi}{|t|}}^{\infty} e^{-i t \tau} \int_{0}^{\frac{\pi}{|t|}} \cdots \int_{0}^{\frac{\pi}{|t|}} F^{(N+1)}\left(\tau+s_{1}+\ldots+s_{N+1}\right) d s_{1} \cdots d s_{N+1} d \tau\right| .
\end{aligned}
$$

Proof. We can write

$$
I(t)=\int_{0}^{\frac{\pi}{|t|}} e^{-i t \tau} F(\tau) d \tau+\int_{\frac{\pi}{|t|}}^{\infty} e^{-i t \tau} F(\tau) d \tau=S+L
$$

Introducing a factor of $e^{i \operatorname{sgn}(t) \pi}$, we can split $L$ as follows:

$$
\begin{aligned}
L & =\frac{1}{2}\left[\int_{\frac{\pi}{|t|}}^{\infty} e^{-i t \tau} F(\tau) d \tau-\int_{\frac{\pi}{|t|}}^{\infty} e^{i \operatorname{sgn}(t) \pi} e^{-i t \tau} F(\tau) d \tau\right] \\
& =\frac{1}{2}\left[\int_{\frac{\pi}{|t|}}^{\infty} e^{-i t \tau} F(\tau) d \tau-\int_{\frac{\pi}{|t|}}^{\infty} e^{-i t\left(\tau-\frac{\pi}{|t|}\right)} F(\tau) d \tau\right] \\
& =\frac{1}{2}\left[\int_{\frac{\pi}{|t|}}^{\infty} e^{-i t \tau} F(\tau) d \tau-\int_{0}^{\infty} e^{-i t \tau} F\left(\tau+\frac{\pi}{|t|}\right) d \tau\right]
\end{aligned}
$$

Writing $F\left(\tau+\frac{\pi}{|t|}\right)=\left[F\left(\tau+\frac{\pi}{|t|}\right)-F(\tau)\right]+F(\tau)$, we have that

$$
L=\frac{1}{2}\left(-\int_{0}^{\frac{\pi}{|t|}} e^{-i t \tau} F(\tau) d \tau-\int_{0}^{\infty} e^{-i t \tau}\left[F\left(\tau+\frac{\pi}{|t|}\right)-F(\tau)\right] d \tau\right)
$$

Using this last expression in equation (56), it follows that

$$
I(t)=\frac{1}{2}\left(\int_{0}^{\frac{\pi}{|t|}} e^{-i t \tau} F(\tau) d \tau-\int_{0}^{\infty} e^{-i t \tau}\left[F\left(\tau+\frac{\pi}{|t|}\right)-F(\tau)\right] d \tau\right) .
$$

Now let $F_{1}(\tau)=F\left(\tau+\frac{\pi}{|t|}\right)-F(\tau)$ and let $I_{1}(t)=\int_{0}^{\infty} e^{-i t \tau} F_{1}(\tau) d \tau$. Then, by the same argument, it follows that

$$
I_{1}(t)=\frac{1}{2}\left(\int_{0}^{\frac{\pi}{|t|}} e^{-i t \tau} F_{1}(\tau) d \tau-\int_{0}^{\infty} e^{-i t \tau}\left[F_{1}\left(\tau+\frac{\pi}{|t|}\right)-F_{1}(\tau)\right] d \tau\right)
$$

After $N$ times of repeating this process, we have that

$$
\begin{aligned}
I_{N}(t) & =\int_{0}^{\infty} e^{-i t \tau} F_{N}(\tau) d \tau \\
& =\frac{1}{2}\left(\int_{0}^{\frac{\pi}{|t|}} e^{-i t \tau} F_{N}(\tau) d \tau-\int_{0}^{\infty} e^{-i t \tau}\left[F_{N}\left(\tau+\frac{\pi}{|t|}\right)-F_{N}(\tau)\right] d \tau\right),
\end{aligned}
$$

where $F_{N}(\tau)=F_{N-1}\left(\tau+\frac{\pi}{|t|}\right)-F_{N-1}(\tau)$.

Letting 


$$
S_{j}(t)=\int_{0}^{\frac{\pi}{|t|}} e^{-i t \tau} F_{j}(\tau) d \tau
$$

for $1 \leq j \leq N-1$, it follows that

$$
I(t)=\frac{1}{2}\left[S(t)-\frac{1}{2}\left[S_{1}(t)-\frac{1}{2}\left[S_{2}(t) \cdots-\frac{1}{2}\left[S_{N-1}(t)-I_{N}(t)\right]\right]\right]\right] .
$$

That is,

$$
I(t)=\frac{1}{2} S(t)+\sum_{k=1}^{N} \frac{(-1)^{k}}{2^{k+1}} \int_{0}^{\frac{\pi}{|t|}} e^{-i t \tau} F_{k}(\tau) d \tau+\frac{(-1)^{N+1}}{2^{N+1}} \int_{0}^{\infty} e^{-i t \tau} F_{N+1}(\tau) d \tau .
$$

Notice that after expanding and rearranging terms, we can write for $1 \leq k \leq N$

$$
F_{k}(\tau)=\sum_{j=0}^{k-1}(-1)^{k+j+1}\left(\begin{array}{c}
k-1 \\
j
\end{array}\right)\left[F\left(\tau+\frac{(j+1) \pi}{|t|}\right)-F\left(\tau+\frac{j \pi}{|t|}\right)\right] .
$$

It follows that

$$
\begin{aligned}
I(t) & =\frac{1}{2} S(t)+\sum_{k=1}^{N} \sum_{j=0}^{k-1}\left(\begin{array}{c}
k-1 \\
j
\end{array}\right) \frac{(-1)^{j+1}}{2^{k+1}} \int_{0}^{\frac{\pi}{|t|}} e^{-i t \tau}\left[F\left(\tau+\frac{(j+1) \pi}{|t|}\right)-F\left(\tau+\frac{j \pi}{|t|}\right)\right] d \tau \\
& +\sum_{j=0}^{N} \frac{(-1)^{j+1}}{2^{N+1}}\left(\begin{array}{c}
N \\
j
\end{array}\right) \int_{0}^{\frac{\pi}{|t|}} e^{-i t \tau}\left[F\left(\tau+\frac{(j+1) \pi}{|t|}\right)-F\left(\tau+\frac{j \pi}{|t|}\right)\right] d \tau \\
& +\frac{(-1)^{N+1}}{2^{N+1}} \int_{\frac{\pi}{|t|}}^{\infty} e^{-i t \tau} F_{N+1}(\tau) d \tau .
\end{aligned}
$$

Changing the order of summation, we get

$$
\begin{aligned}
I(t) & =\frac{1}{2} S(t)+\sum_{j=1}^{N} \sum_{k=j}^{N}\left(\begin{array}{c}
k-1 \\
j-1
\end{array}\right) \frac{(-1)^{j}}{2^{k+1}} \int_{0}^{\frac{\pi}{|t|}} e^{-i t \tau} F\left(\tau+\frac{j \pi}{|t|}\right) d \tau \\
& +\sum_{j=0}^{N-1} \sum_{k=j+1}^{N}\left(\begin{array}{c}
k-1 \\
j
\end{array}\right) \frac{(-1)^{j}}{2^{k+1}} \int_{0}^{\frac{\pi}{|t|}} e^{-i t \tau} F\left(\tau+\frac{j \pi}{t}\right) d \tau \\
& +\sum_{j=1}^{N+1} \frac{(-1)^{j}}{2^{N+1}}\left(\begin{array}{c}
N \\
j-1
\end{array}\right) \int_{0}^{\frac{\pi}{|t|}} e^{-i t \tau} F\left(\tau+\frac{j \pi}{|t|}\right) d \tau \\
& +\sum_{j=0}^{N} \frac{(-1)^{j}}{2^{N+1}}\left(\begin{array}{c}
N \\
j
\end{array}\right) \int_{0}^{\frac{\pi}{|t|}} e^{-i t \tau} F\left(\tau+\frac{j \pi}{|t|}\right) d \tau+\frac{(-1)^{N+1}}{2^{N+1}} \int_{\frac{\pi}{|t|}}^{\infty} e^{-i t \tau} F_{N+1}(\tau) d \tau .
\end{aligned}
$$

\section{Letting}




$$
\begin{aligned}
& c_{0}=\frac{1}{2}+\sum_{k=1}^{N} \frac{1}{2^{k+1}}+\frac{1}{2^{N+1}} \\
& c_{j}=\sum_{k=j}^{N}\left(\begin{array}{c}
k-1 \\
j-1
\end{array}\right) \frac{1}{2^{k+1}}+\sum_{k=j+1}^{N}\left(\begin{array}{c}
k-1 \\
j
\end{array}\right) \frac{1}{2^{k+1}}+\left(\begin{array}{c}
N \\
j-1
\end{array}\right) \frac{1}{2^{N+1}}+\left(\begin{array}{c}
N \\
j
\end{array}\right) \frac{1}{2^{N+1}} \text { for } 1 \leq J \leq N-1 ; \\
& c_{N}=\frac{N+2}{2^{N+1}} ; \text { and } \\
& c_{N+1}=\frac{1}{2^{N+1}},
\end{aligned}
$$

it follows that

$$
|I(t)| \leq \sum_{j=0}^{N+1} c_{j}\left|\int_{0}^{\frac{\pi}{|t|}} e^{-i t \tau} F\left(\tau+\frac{j \pi}{|t|}\right) d \tau\right|+\frac{1}{2^{N+1}}\left|\int_{0}^{\infty} e^{-i t \tau} F_{N+1}(\tau) d \tau\right|
$$

It is worth noting that the exact form of the coefficients $c_{k}$ for $1 \leq k \leq N$ is irrelevant. The only fact that will be needed is that they exist and are positive.

It suffices now to show that

$$
F_{N+1}(\tau)=\int_{0}^{\frac{\pi}{|t|}} \cdots \int_{0}^{\frac{\pi}{|t|}} F^{(N+1)}\left(\tau+s_{1}+\ldots+s_{N+1}\right) d s_{1} \cdots d s_{N+1}
$$

where

$$
\begin{aligned}
F_{N+1}(\tau) & =\sum_{j=0}^{N}(-1)^{N+j}\left(\begin{array}{c}
N \\
j
\end{array}\right)\left[F\left(\tau+\frac{(j+1) \pi}{|t|}\right)-F\left(\tau+\frac{j \pi}{|t|}\right)\right] \\
& =\sum_{j=0}^{N}(-1)^{N+j}\left(\begin{array}{c}
N \\
j
\end{array}\right) \int_{0}^{\frac{\pi}{|t|}} F^{\prime}\left(\tau+s+\frac{j \pi}{|t|}\right) d s .
\end{aligned}
$$

Using the identity

$$
\left(\begin{array}{l}
n \\
k
\end{array}\right)=\left(\begin{array}{c}
n-1 \\
k
\end{array}\right)+\left(\begin{array}{l}
n-1 \\
k-1
\end{array}\right)
$$

it follows that for any integer $M>0$ and for any function $h$,

$$
\sum_{j=0}^{M}(-1)^{M+j}\left(\begin{array}{c}
M \\
j
\end{array}\right) h(j)=\sum_{j=0}^{M-1}(-1)^{M+j+1}\left(\begin{array}{c}
M-1 \\
j
\end{array}\right)[h(j+1)-h(j)] .
$$

Let $h(j)=\int_{0}^{\frac{\pi}{|t|}} F^{\prime}\left(\tau+s+\frac{j \pi}{|t|}\right) d s$ and $M=N$. Notice that we can write

$$
h(j+1)-h(j)=\int_{0}^{\frac{\pi}{|t|}} \int_{0}^{\frac{\pi}{|t|}} F^{\prime \prime}\left(\tau+s_{1}+s_{2}+\frac{j \pi}{|t|}\right) d s_{1} d s_{2} .
$$

It follows from equations (58), (59) and (60) that 


$$
F_{N+1}(\tau)=\sum_{j=0}^{N-1}(-1)^{N+j+1}\left(\begin{array}{c}
N-1 \\
j
\end{array}\right) \int_{0}^{\frac{\pi}{|t|}} \int_{0}^{\frac{\pi}{|t|}} F^{\prime \prime}\left(\tau+s_{1}+s_{2}+\frac{j \pi}{|t|}\right) d s_{1} d s_{2} .
$$

Repeating this process $N-1$ times, where the $i^{\text {th }}$ time we choose

$$
h(j)=\int_{0}^{\frac{\pi}{|t|}} \int_{0}^{\frac{\pi}{|t|}} F^{(i+1)}\left(\tau+s_{1}+\ldots+s_{i+1}+\frac{j \pi}{|t|}\right) d s_{1} \ldots d s_{i+1}
$$

and $M=N-i$, we obtain

$$
F_{N+1}(\tau)=\int_{0}^{\frac{\pi}{|t|}} \cdots \int_{0}^{\frac{\pi}{|t|}} F^{(N+1)}\left(\tau+s_{1}+\ldots+s_{N+1}\right) d s_{1} \cdots d s_{N+1}
$$

This finishes the proof of Claim 5.9.

Claim 5.10. The $N^{\text {th }}$ derivative of

$$
F(\tau)=e^{-2 \pi \tau \delta} \int_{\mathbb{R}^{n}} \frac{e^{2 \pi i \boldsymbol{\eta} \cdot\left(\boldsymbol{y}-\boldsymbol{y}^{\prime}\right)}}{\int_{\mathbb{R}^{n}} e^{4 \pi[\boldsymbol{\eta} \cdot \boldsymbol{v}-\tau \tilde{b}(\boldsymbol{v})]} d \boldsymbol{v}} d \boldsymbol{\eta}
$$

consists of sums of terms of the form

$$
\frac{C(\tau \delta)^{N-k} e^{-2 \pi \tau \delta}}{\tau^{N}} \int_{\mathbb{R}^{n}} \frac{e^{2 \pi i \boldsymbol{\eta} \cdot\left(\boldsymbol{y}-\boldsymbol{y}^{\prime}\right)} f_{1}(\tau, \boldsymbol{\eta}) \cdots f_{k}(\tau, \boldsymbol{\eta})}{\gamma(\tau, \boldsymbol{\eta})} d \boldsymbol{\eta},
$$

where

$$
\begin{gathered}
f_{s}(\tau, \boldsymbol{\eta})=\left[\int_{\mathbb{R}^{n}}(\tau \tilde{b}(\boldsymbol{v}))^{s} e^{4 \pi[\boldsymbol{\eta} \cdot \boldsymbol{v}-\tau \tilde{b}(\boldsymbol{v})]} d \boldsymbol{v}\right]^{a_{s}} ; \\
\gamma(\tau, \boldsymbol{\eta})=\left[\int_{\mathbb{R}^{n}} e^{4 \pi[\boldsymbol{\eta} \cdot \boldsymbol{v}-\tau \tilde{b}(\boldsymbol{v})]} d \boldsymbol{v}\right]^{d} ;
\end{gathered}
$$

$a_{1}, \ldots, a_{k}, k, d \in \mathbb{N} ; 0 \leq k \leq N ; a_{1}+\ldots+a_{k}=d-1 ;$ and $a_{1}+2 a_{2}+\ldots+k a_{k}=k$.

Proof. We will begin by showing by induction that the $k^{\text {th }}$ derivative of

$$
J(\tau)=\int_{\mathbb{R}^{n}} \frac{e^{2 \pi i \boldsymbol{\eta} \cdot\left(\boldsymbol{y}-\boldsymbol{y}^{\prime}\right)}}{\int_{\mathbb{R}^{n}} e^{4 \pi[\boldsymbol{\eta} \cdot \boldsymbol{v}-\tau \tilde{b}(\boldsymbol{v})]} d \boldsymbol{v}} d \boldsymbol{\eta}
$$

consists of sums of terms of the form

$$
C \int_{\mathbb{R}^{n}} \frac{e^{2 \pi i \boldsymbol{\eta} \cdot\left(\boldsymbol{y}-\boldsymbol{y}^{\prime}\right)}\left[\int_{\mathbb{R}^{n}} \tilde{b}(\boldsymbol{v}) e^{4 \pi[\boldsymbol{\eta} \cdot \boldsymbol{v}-\tau \tilde{b}(\boldsymbol{v})]} d \boldsymbol{v}\right]^{a_{1}} \cdots\left[\int_{\mathbb{R}^{n}} \tilde{b}(\boldsymbol{v})^{k} e^{4 \pi[\boldsymbol{\eta} \cdot \boldsymbol{v}-\tau \tilde{b}(\boldsymbol{v})]} d \boldsymbol{v}\right]^{a_{k}}}{\left[\int_{\mathbb{R}^{n}} e^{4 \pi[\boldsymbol{\eta} \cdot \boldsymbol{v}-\tau \tilde{b}(\boldsymbol{v})]} d \boldsymbol{v}\right]^{d}} d \boldsymbol{\eta},
$$

where $a_{1}+\ldots+a_{k}=d-1$; and $a_{1}+2 a_{2}+\ldots+k a_{k}=k$. 
Notice that $J^{\prime}(\tau)$ is of this form, with $a_{1}=1$, and $d=2$. Suppose $J^{(k)}(\tau)$ is of this form. We will show that $J^{(k+1)}(\tau)$ is of this form. Let

$$
g_{s}(\tau)=\left[\int_{\mathbb{R}^{n}} \tilde{b}(\boldsymbol{v})^{s} e^{4 \pi[\boldsymbol{\eta} \cdot \boldsymbol{v}-\tau \tilde{b}(\boldsymbol{v})]} d \boldsymbol{v}\right]^{a_{s}}
$$

Then $\frac{d}{d \tau}\left[J^{(k)}(\tau)\right]$ consists of sums of terms of the form

$$
C \int_{\mathbb{R}^{n}} \frac{e^{2 \pi i \boldsymbol{\eta} \cdot\left(\boldsymbol{y}-\boldsymbol{y}^{\prime}\right)} g_{1} \cdots g_{s-1} \frac{d}{d \tau}\left(g_{s}\right) g_{s+1} \cdots g_{k}}{\gamma} d \boldsymbol{\eta}
$$

or

$$
C \int_{\mathbb{R}^{n}} \frac{e^{2 \pi i \boldsymbol{\eta} \cdot\left(\boldsymbol{y}-\boldsymbol{y}^{\prime}\right)} g_{1} \cdots g_{k} \frac{d}{d \tau}(\gamma)}{\gamma^{2}} d \boldsymbol{\eta}
$$

with $\gamma$ as in equation (62). But

$$
\frac{d}{d \tau}\left(g_{s}\right)=-4 \pi a_{s}\left[\int_{\mathbb{R}^{n}} \tilde{b}(\boldsymbol{v})^{s} e^{4 \pi[\boldsymbol{\eta} \cdot \boldsymbol{v}-\tau \tilde{b}(\boldsymbol{v})]} d \boldsymbol{v}\right]^{a_{s}-1}\left[\int_{\mathbb{R}^{n}} \tilde{b}(\boldsymbol{v})^{s+1} e^{4 \pi[\boldsymbol{\eta} \cdot \boldsymbol{v}-\tau \tilde{b}(\boldsymbol{v})]} d \boldsymbol{v}\right]
$$

and

$$
\frac{d}{d \tau}(\gamma)=-4 \pi d \gamma^{\frac{d-1}{d}} \int_{\mathbb{R}^{n}} \widetilde{b}(\boldsymbol{v}) e^{4 \pi[\boldsymbol{\eta} \cdot \boldsymbol{v}-\tau \tilde{b}(\boldsymbol{v})]} d \boldsymbol{v}
$$

Thus, a generic term of the form given in equation (63) is given by

$$
C \int_{\mathbb{R}^{n}} \frac{e^{2 \pi i \boldsymbol{\eta} \cdot\left(\boldsymbol{y}-\boldsymbol{y}^{\prime}\right)} g_{1} \cdots g_{k}}{\gamma}\left[\int_{\mathbb{R}^{n}} \tilde{b}(\boldsymbol{v})^{s} e^{4 \pi[\boldsymbol{\eta} \cdot \boldsymbol{v}-\tau \tilde{b}(\boldsymbol{v})]} d \boldsymbol{v}\right]^{-1}\left[\int_{\mathbb{R}^{n}} \tilde{b}(\boldsymbol{v})^{s+1} e^{4 \pi[\boldsymbol{\eta} \cdot \boldsymbol{v}-\tau \tilde{b}(\boldsymbol{v})]} d \boldsymbol{v}\right] d \boldsymbol{\eta} \text {. }
$$

Let $h_{j}=g_{j}$ for $j \neq s, s+1 ; h_{s}=\left[\int_{\mathbb{R}^{n}} \widetilde{b}(\boldsymbol{v})^{s} e^{4 \pi[\boldsymbol{\eta} \cdot \boldsymbol{v}-\tau \tilde{b}(\boldsymbol{v})]} d \boldsymbol{v}\right]^{\widetilde{a}_{s}}$, where $\widetilde{a}_{s}=a_{s}-1 ;$ and $h_{s+1}=$ $\left[\int_{\mathbb{R}^{n}} \widetilde{b}(\boldsymbol{v})^{s+1} e^{4 \pi[\boldsymbol{\eta} \cdot \boldsymbol{v}-\tau \tilde{b}(\boldsymbol{v})]} d \boldsymbol{v}\right]^{\widetilde{a}_{s+1}}$, where $\widetilde{a}_{s+1}=a_{s+1}+1$. Then equation $(65)$ can be written as

$$
C \int_{\mathbb{R}^{n}} \frac{e^{2 \pi i \boldsymbol{\eta} \cdot\left(\boldsymbol{y}-\boldsymbol{y}^{\prime}\right)} h_{1} \cdots h_{k}}{\gamma} d \boldsymbol{\eta} .
$$

For this term to have the desired form, the exponents must satisfy $a_{1}+\ldots+a_{s-1}+\widetilde{a}_{s}+\widetilde{a}_{s+1}+$ $a_{s+2}+\ldots+a_{k}=d-1$, and $a_{1}+\ldots+(s-1) a_{s-1}+s \widetilde{a}_{s}+(s+1) \widetilde{a}_{s+1}+(s+2) a_{s+2}+\ldots+k a_{k}=k+1$. The former holds, since by inductive hypothesis $a_{1}+\ldots+a_{s-1}+\widetilde{a}_{s}+\widetilde{a}_{s+1}+a_{s+2}+\ldots+a_{k}=$ $a_{1}+\ldots+a_{s}-1+a_{s}+1+\ldots+a_{k}=a_{1}+\ldots+a_{k}=d-1$. The latter also holds, since by inductive hypothesis, $a_{1}+\ldots+(s-1) a_{s-1}+s \widetilde{a}_{s}+(s+1) \widetilde{a}_{s+1}+(s+2) a_{s+2}+\ldots+k a_{k}=a_{1}+\ldots+(s-$ 1) $a_{s-1}+s a_{s}-s+(s+1) a_{s+1}+s+1+(s+2) a_{s+2}+\ldots+k a_{k}=a_{1}+\ldots+k a_{k}+1=k+1$.

In the same way, a generic term of the form given in equation (64) is given by 


$$
C \int_{\mathbb{R}^{n}} \frac{e^{2 \pi i \boldsymbol{\eta} \cdot\left(\boldsymbol{y}-\boldsymbol{y}^{\prime}\right)} g_{1} \cdots g_{k} \int_{\mathbb{R}^{n}} \tilde{b}(\boldsymbol{v}) e^{4 \pi[\boldsymbol{\eta} \cdot \boldsymbol{v}-\tau \tilde{b}(\boldsymbol{v})]} d \boldsymbol{v}}{\left[\int_{\mathbb{R}^{n}} e^{4 \pi[\boldsymbol{\eta} \cdot \boldsymbol{v}-\tau \tilde{b}(\boldsymbol{v})]} d \boldsymbol{v}\right]^{d+1}} d \boldsymbol{\eta} .
$$

Let $h_{j}=g_{j}$ for $j \neq 1$, and $h_{1}=\left[\int_{\mathbb{R}^{n}} \widetilde{b}(\boldsymbol{v}) e^{4 \pi[\boldsymbol{\eta} \cdot \boldsymbol{v}-\tau \tilde{b}(\boldsymbol{v})]} d \boldsymbol{v}\right]^{\widetilde{a}_{1}}$, where $\widetilde{a}_{1}=a_{1}+1$ so that equation (66) can be written as

$$
C \int_{\mathbb{R}^{n}} \frac{e^{2 \pi i \boldsymbol{\eta} \cdot\left(\boldsymbol{y}-\boldsymbol{y}^{\prime}\right)} h_{1} \cdots h_{k}}{\left[\int_{\mathbb{R}^{n}} e^{4 \pi[\boldsymbol{\eta} \cdot \boldsymbol{v}-\tau \tilde{b}(\boldsymbol{v})]} d \boldsymbol{v}\right]^{d+1}} d \boldsymbol{\eta} .
$$

For this term to have the desired form, the exponents must satisfy $\widetilde{a}_{1}+a_{2}+\ldots+a_{k}=d$, and $\tilde{a}_{1}+2 a_{2}+\ldots+k a_{k}=k+1$. The former holds, since by inductive hypothesis, $\widetilde{a}_{1}+a_{2}+\ldots+$ $a_{k}=a_{1}+\ldots+a_{k}+1=(d-1)+1=d$. The latter also holds, since by inductive hypothesis $\widetilde{a}_{1}+2 a_{2}+\ldots+k a_{k}=a_{1}+2 a_{2}+\ldots+k a_{k}+1=k+1$.

It follows that for any $k \in \mathbb{N}$, the $k^{t h}$ derivative of $J(\tau)$ consists of sums of terms of the form

$$
C \int_{\mathbb{R}^{n}} \frac{e^{2 \pi i \boldsymbol{\eta} \cdot\left(\boldsymbol{y}-\boldsymbol{y}^{\prime}\right)}\left[\int_{\mathbb{R}^{n}} \tilde{b}(\boldsymbol{v}) e^{4 \pi[\boldsymbol{\eta} \cdot \boldsymbol{v}-\tau \tilde{b}(\boldsymbol{v})]} d \boldsymbol{v}\right]^{a_{1}} \cdots\left[\int_{\mathbb{R}^{n}} \tilde{b}(\boldsymbol{v})^{k} e^{4 \pi[\boldsymbol{\eta} \cdot \boldsymbol{v}-\tau \tilde{b}(\boldsymbol{v})]} d \boldsymbol{v}\right]^{a_{k}}}{\left[\int_{\mathbb{R}^{n}} e^{4 \pi[\boldsymbol{\eta} \cdot \boldsymbol{v}-\tau \tilde{b}(\boldsymbol{v})]} d \boldsymbol{v}\right]^{d}} d \boldsymbol{\eta},
$$

where $a_{1}+\ldots+a_{k}=d-1 ;$ and $a_{1}+2 a_{2}+\ldots+k a_{k}=k$.

Finally, since $F(\tau)=e^{-2 \pi \tau \delta} J(\tau)$, the $N^{t h}$ derivative of $F$ is given by

$$
F^{(N)}(\tau)=\sum_{k=0}^{N}\left(\begin{array}{l}
N \\
k
\end{array}\right)\left(e^{-2 \pi \tau \delta}\right)^{(N-k)} J^{(k)}(\tau) .
$$

But $\left(e^{-2 \pi \tau \delta}\right)^{(N-k)}=C \delta^{N-k} e^{-2 \pi \tau \delta}$. Thus, the $N^{t h}$ derivative of $F$ consists of sums of multiples of terms of the form

$$
\delta^{N-k} e^{-2 \pi \tau \delta} \int_{\mathbb{R}^{n}} \frac{e^{2 \pi i \boldsymbol{\eta} \cdot\left(\boldsymbol{y}-\boldsymbol{y}^{\prime}\right)}\left[\int_{\mathbb{R}^{n}} \tilde{b}(\boldsymbol{v}) e^{4 \pi[\boldsymbol{\eta} \cdot \boldsymbol{v}-\tau \tilde{b}(\boldsymbol{v})]} d \boldsymbol{v}\right]^{a_{1}} \cdots\left[\int_{\mathbb{R}^{n}} \tilde{b}(\boldsymbol{v})^{k} e^{4 \pi[\boldsymbol{\eta} \cdot \boldsymbol{v}-\tau \tilde{b}(\boldsymbol{v})]} d \boldsymbol{v}\right]^{a_{k}}}{\left[\int_{\mathbb{R}^{n}} e^{4 \pi[\boldsymbol{\eta} \cdot \boldsymbol{v}-\tau \tilde{b}(\boldsymbol{v})]} d \boldsymbol{v}\right]^{d}} d \boldsymbol{\eta},
$$

where $a_{1}+\ldots+a_{k}=d-1$ and $a_{1}+2 a_{2}+\ldots+k a_{k}=k$.

Finally, writing for $1 \leq s \leq k$,

$$
\left[\int_{\mathbb{R}^{n}} \widetilde{b}(\boldsymbol{v})^{s} e^{4 \pi[\boldsymbol{\eta} \cdot \boldsymbol{v}-\tau \tilde{b}(\boldsymbol{v})]} d \boldsymbol{v}\right]^{a_{s}}=\frac{1}{\tau^{s a_{s}}}\left[\int_{\mathbb{R}^{n}}(\tau \tilde{b}(\boldsymbol{v}))^{s} e^{4 \pi[\boldsymbol{\eta} \cdot \boldsymbol{v}-\tau \tilde{b}(\boldsymbol{v})]} d \boldsymbol{v}\right]^{a_{s}},
$$

yields the desired expression. This finishes the proof of Claim 5.10. 
Claim 5.11. Let

$$
\Delta_{N, k}^{\boldsymbol{\mu}}(\tau)=(\tau \delta)^{N-k} e^{-2 \pi \tau \delta} \int_{\mathbb{R}^{n}} \frac{e^{2 \pi i \frac{\boldsymbol{\eta}}{\boldsymbol{\mu}} \cdot\left(\boldsymbol{y}-\boldsymbol{y}^{\prime}\right)} f_{1}^{\mu}(\tau, \boldsymbol{\eta}) \cdots f_{k}^{\mu}(\tau, \boldsymbol{\eta})}{\gamma^{\mu}(\tau, \boldsymbol{\eta})} d \boldsymbol{\eta},
$$

where

$$
\begin{gathered}
f_{s}^{\mu}(\tau, \boldsymbol{\eta})=\left[\int_{\mathbb{R}^{n}}(\tau \tilde{b}(\boldsymbol{\mu} \boldsymbol{v}))^{s} e^{4 \pi[\boldsymbol{\eta} \cdot \boldsymbol{v}-\tau \tilde{b}(\boldsymbol{\mu} \boldsymbol{v})]} d \boldsymbol{v}\right]^{a_{s}} ; \\
\gamma^{\mu}(\tau, \boldsymbol{\eta})=\left[\int_{\mathbb{R}^{n}} e^{4 \pi[\boldsymbol{\eta} \cdot \boldsymbol{v}-\tau \tilde{b}(\boldsymbol{\mu} \boldsymbol{v})]} d \boldsymbol{v}\right]^{d}
\end{gathered}
$$

$a_{1}, \ldots, a_{k}, k, d \in \mathbb{N} ; 0 \leq k \leq N ; a_{1}+\ldots+a_{k}=d-1 ;$ and $a_{1}+2 a_{2}+\ldots+k a_{k}=k$. Then there exists a constant $C$ that depends only on $N, k$, the combined degree of $b$ and the dimension of the space such that

$$
\left|\Delta_{N, k}^{\mu}(\tau)\right| \leq C
$$

Remark 5.12. Notice that $\mu$ is a function of $\tau$.

Proof. It is easy to check that $(\tau \delta)^{N-k} e^{-2 \pi \tau \delta}$ is bounded. Thus, it suffices to show that

$$
\int_{\mathbb{R}^{n}} \frac{f_{1}^{\mu}(\tau, \boldsymbol{\eta}) \cdots f_{k}^{\mu}(\tau, \boldsymbol{\eta})}{\gamma^{\mu}(\tau, \boldsymbol{\eta})} d \boldsymbol{\eta}
$$

is bounded. We will begin by studying the properties of the $f_{s}^{\mu}(\tau, \boldsymbol{\eta})$ defined above. By Claim 4.3 on page 12 there exists a universal constant such that

$$
\tau \widetilde{b}(\boldsymbol{\mu v}) \leq C\left(1+v_{1}^{2 m_{1}}+\ldots+v_{n}^{2 m_{n}}\right) .
$$

Thus, we must study the behavior of integrals of the form

$$
L_{s}=\int_{\mathbb{R}^{n}} p_{s}(\boldsymbol{v}) e^{\eta \cdot \boldsymbol{v}-g(\boldsymbol{v})} d \boldsymbol{v},
$$

for polynomials $p_{s}: \mathbb{R}^{n} \rightarrow \mathbb{R}^{+}$with non-negative coefficients and $g(\boldsymbol{v})=\tau \tilde{b}(\boldsymbol{\mu} \boldsymbol{v})$. As usual (see, e.g., equation (16) on page 11), we can write

$$
L_{s}=e^{h\left(\boldsymbol{v}_{\mathbf{0}}\right)} \int_{\mathbb{R}^{n}} e^{h(\boldsymbol{v})-h\left(\boldsymbol{v}_{\mathbf{0}}\right)} p_{s}(\boldsymbol{v}) d \boldsymbol{v},
$$

where $h(\boldsymbol{v})=\boldsymbol{\eta} \cdot \boldsymbol{v}-g(\boldsymbol{v})$ and $\boldsymbol{v}_{\mathbf{0}}$ is the point where $h(\boldsymbol{v})$ attains its maximum. Making the change of variables $\boldsymbol{v}=\boldsymbol{w}+\boldsymbol{v}_{\mathbf{0}}$, it follows that

$$
L_{s}=e^{h\left(\boldsymbol{v}_{\mathbf{0}}\right)} \int_{\mathbb{R}^{n}} e^{-f(\boldsymbol{w})} p_{s}\left(\boldsymbol{w}+\boldsymbol{v}_{\mathbf{0}}\right) d \boldsymbol{w},
$$

where $f(\boldsymbol{w})=g\left(\boldsymbol{v}_{\mathbf{0}}+\boldsymbol{w}\right)-g\left(\boldsymbol{v}_{\mathbf{0}}\right)-\nabla g\left(\boldsymbol{v}_{\mathbf{0}}\right) \cdot \boldsymbol{w}$. Since the coefficients of $p$ are non-negative, it follows that

$$
p_{s}\left(w_{1}+v_{01}, \ldots, w_{n}+v_{0 n}\right) \leq p_{s}\left(|\boldsymbol{w}|+\left|\boldsymbol{v}_{\mathbf{0}}\right|, \ldots,|\boldsymbol{w}|+\left|\boldsymbol{v}_{\mathbf{0}}\right|\right)
$$


This last polynomial is now a polynomial of just one variable, and after expanding and regrouping all the terms, it consists of sums of terms of the form $|\boldsymbol{w}|^{j_{s}}\left|\boldsymbol{v}_{\mathbf{0}}\right|^{i_{s}}$ for indices $i_{s}$ and $j_{s}$. Hence, there exist positive coefficients $c_{i_{s}, j_{s}}$ such that

$$
L_{s} \leq e^{h\left(\boldsymbol{v}_{\mathbf{0}}\right)} \sum_{i_{s}, j_{s}} c_{i_{s}, j_{s}}\left|\boldsymbol{v}_{\mathbf{0}}\right|^{i_{s}} \int_{\mathbb{R}^{n}}|\boldsymbol{w}|^{j_{s}} e^{-f(\boldsymbol{w})} d \boldsymbol{w}
$$

Let $J_{j_{s}}=\int_{\mathbb{R}^{n}}|\boldsymbol{w}|^{j_{s}} e^{-f(\boldsymbol{w})} d \boldsymbol{w}$. This integral is identical to the one studied in equation (33) in Claim 4.11 on page 20 . Thus,

$$
J_{j_{s}} \lesssim|\{\boldsymbol{w}: f(\boldsymbol{w}) \leq 1\}|\left(1+\left|\boldsymbol{v}_{\mathbf{0}}\right|^{j_{s} B}\right)+\Theta
$$

where $\Theta$ is a constant that depends only on $m_{1}, \ldots, m_{n}$ and the dimension of the space; and $B=$ $4 \max \left\{m_{1}, \ldots, m_{n}\right\}$. It follows that

$$
L_{s} \lesssim e^{h\left(\boldsymbol{v}_{\mathbf{0}}\right)} \sum_{i_{s}, j_{s}} c_{i_{s}, j_{s}}\left|\boldsymbol{v}_{\mathbf{0}}\right|^{i_{s}}\left[|\{\boldsymbol{w}: f(\boldsymbol{w}) \leq 1\}|\left(1+\left|\boldsymbol{v}_{\mathbf{0}}\right|^{j_{s} B}\right)+\Theta\right] .
$$

That is,

$$
L_{s} \lesssim e^{h\left(\boldsymbol{v}_{\mathbf{0}}\right)}\left[|\{\boldsymbol{w}: f(\boldsymbol{w}) \leq 1\}| \phi_{s}\left(\left|\boldsymbol{v}_{\mathbf{0}}\right|\right)+\psi_{s}\left(\left|\boldsymbol{v}_{\mathbf{0}}\right|\right)\right]
$$

for some polynomials $\phi_{s}: \mathbb{R} \rightarrow \mathbb{R}^{+}, \psi_{s}: \mathbb{R} \rightarrow \mathbb{R}^{+}$.

On the other hand, by equation (24)

$$
\int_{\mathbb{R}^{n}} e^{4 \pi[\boldsymbol{\eta} \cdot \boldsymbol{v}-\tau \tilde{b}(\boldsymbol{\mu} \boldsymbol{v})]} d \boldsymbol{v} \approx e^{h\left(\boldsymbol{v}_{\mathbf{0}}\right)}|\{\boldsymbol{w}: f(\boldsymbol{w}) \leq 1\}| .
$$

Therefore, there exist some polynomials $q_{j}: \mathbb{R} \rightarrow \mathbb{R}^{+}$such that

$$
\int_{\mathbb{R}^{n}} \frac{f_{1}^{\mu}(\tau, \boldsymbol{\eta}) \cdots f_{k}^{\mu}(\tau, \boldsymbol{\eta})}{\gamma^{\mu}(\tau, \boldsymbol{\eta})} d \boldsymbol{\eta} \lesssim \int_{\mathbb{R}^{n}} \frac{\left[e^{h\left(\boldsymbol{v}_{\mathbf{0}}\right)}\right]^{a_{1}+\ldots+a_{k}}\left(\sum_{j=0}^{a_{1}+\ldots+a_{k}}|\{\boldsymbol{w}: f(\boldsymbol{w}) \leq 1\}|^{j} q_{j}\left(\left|\boldsymbol{v}_{\mathbf{0}}\right|\right)\right)}{\left[e^{h\left(\boldsymbol{v}_{\mathbf{0}}\right)}\right]^{d}|\{\boldsymbol{w}: f(\boldsymbol{w}) \leq 1\}|^{d}} d \boldsymbol{\eta} .
$$

But $a_{1}+\ldots+a_{k}=d-1$, so

$$
\int_{\mathbb{R}^{n}} \frac{f_{1}^{\mu}(\tau, \boldsymbol{\eta}) \cdots f_{k}^{\mu}(\tau, \boldsymbol{\eta})}{\gamma^{\mu}(\tau, \boldsymbol{\eta})} d \boldsymbol{\eta} \lesssim \int_{\mathbb{R}^{n}} e^{-h\left(\boldsymbol{v}_{\mathbf{0}}\right)}\left(\sum_{j=0}^{d-1}|\{\boldsymbol{w}: f(\boldsymbol{w}) \leq 1\}|^{j-d} q_{j}\left(\left|\boldsymbol{v}_{\mathbf{0}}\right|\right)\right) d \boldsymbol{\eta} .
$$

Moreover, by Claim 4.7,

$$
|\{\boldsymbol{w}: f(\boldsymbol{w}) \leq 1\}| \gtrsim\left(1+r\left(\boldsymbol{v}_{\mathbf{0}}\right)\right)^{-\frac{n}{2}} .
$$

where $r(\boldsymbol{v})=v_{1}^{2 m_{1}}+\ldots+v_{n}^{2 m_{n}}$. Thus, and since $j-d<0$,

$$
\int_{\mathbb{R}^{n}} \frac{f_{1}^{\mu}(\tau, \boldsymbol{\eta}) \cdots f_{k}^{\mu}(\tau, \boldsymbol{\eta})}{\gamma^{\mu}(\tau, \boldsymbol{\eta})} d \boldsymbol{\eta} \lesssim \int_{\mathbb{R}^{n}} e^{-h\left(\boldsymbol{v}_{\mathbf{0}}\right)}\left(\sum_{j=0}^{d-1}\left(1+r\left(\boldsymbol{v}_{\mathbf{0}}\right)\right)^{\frac{n(d-j)}{2}} q_{j}\left(\left|\boldsymbol{v}_{\mathbf{0}}\right|\right)\right) d \boldsymbol{\eta} .
$$

By Claims 4.9 and $4.8, \sum_{j=0}^{d-1}\left(1+r\left(\boldsymbol{v}_{\mathbf{0}}\right)\right)^{\frac{n(d-j)}{2}} q_{j}\left(\left|\boldsymbol{v}_{\mathbf{0}}\right|\right)$ is at most of polynomial growth in $|\boldsymbol{\eta}|$. On the other hand, by equation (21), $e^{-h\left(\boldsymbol{v}_{\mathbf{0}}\right)}$ decays exponentially in $|\boldsymbol{\eta}|$. Hence, 


$$
\int_{\mathbb{R}^{n}} \frac{f_{1}^{\mu}(\tau, \boldsymbol{\eta}) \cdots f_{k}^{\mu}(\tau, \boldsymbol{\eta})}{\gamma^{\mu}(\tau, \boldsymbol{\eta})} d \boldsymbol{\eta}
$$

is bounded. This finishes the proof of Claim 5.11.

Corollary 5.13. Let

$$
F(\tau)=e^{-2 \pi \tau \delta} \int_{\mathbb{R}^{n}} \frac{e^{2 \pi i \boldsymbol{\eta} \cdot\left(\boldsymbol{y}-\boldsymbol{y}^{\prime}\right)}}{\int_{\mathbb{R}^{n}} e^{4 \pi[\boldsymbol{\eta} \cdot \boldsymbol{v}-\tau \tilde{b}(\boldsymbol{v})]} d \boldsymbol{v}} d \boldsymbol{\eta} .
$$

Then $F \in L^{1}(\mathbb{R})$.

Proof. Making the change of variables $\boldsymbol{\eta} \rightarrow \frac{\eta}{\mu}$ and $\boldsymbol{v} \rightarrow \boldsymbol{\mu v}$, we have that

$$
F(\tau)=\frac{e^{-2 \pi \tau \delta}}{\mu_{1}^{2} \cdots \mu_{n}^{2}} \int_{\mathbb{R}^{n}} \frac{e^{2 \pi i \frac{\boldsymbol{\eta}}{\boldsymbol{\mu}} \cdot\left(\boldsymbol{y}-\boldsymbol{y}^{\prime}\right)}}{\int_{\mathbb{R}^{n}} e^{4 \pi[\boldsymbol{\eta} \cdot \boldsymbol{v}-\tau \tilde{b}(\boldsymbol{\mu} \boldsymbol{v})]} d \boldsymbol{v}} d \boldsymbol{\eta} .
$$

Taking $k=N=0$, and $d=1$, it follows from the proof of Claim 5.11 that

$$
\left|\int_{\mathbb{R}^{n}} \frac{e^{2 \pi i \frac{\boldsymbol{\eta}}{\boldsymbol{\mu}} \cdot\left(\boldsymbol{y}-\boldsymbol{y}^{\prime}\right)}}{\int_{\mathbb{R}^{n}} e^{4 \pi[\boldsymbol{\eta} \cdot \boldsymbol{v}-\tau \tilde{b}(\boldsymbol{\mu} \boldsymbol{v})]} d \boldsymbol{v}} d \boldsymbol{\eta}\right|
$$

is bounded. Thus, and by equation (47), it follows that

$$
|F(\tau)| \lesssim \frac{e^{-2 \pi \tau \delta}}{\left|\left\{\boldsymbol{v}: \widetilde{b}(\boldsymbol{v}) \leq \frac{1}{\tau}\right\}\right|^{2}}
$$

If $\tau \leq 1$, then $|F(\tau)|$ is bounded. If $\tau>1$, it follows from Claim 6.1 (see Appendix) that

which decays exponentially as $\tau \rightarrow \infty$.

$$
|F(\tau)| \lesssim \frac{e^{-2 \pi \tau \delta} \tau^{2 n}}{|\{\boldsymbol{v}: \widetilde{b}(\boldsymbol{v}) \leq 1\}|^{2}}
$$

We are now ready to present the proof of Proposition 5.8.

Proof. We had shown in equation (48) on page 27 that

$$
S=\int_{0}^{\infty} e^{-i u \tau} F(\tau) d \tau
$$

where $u=2 \pi w$, and

$$
F(\tau)=e^{-2 \pi \tau \delta} \int_{\mathbb{R}^{n}} \frac{e^{2 \pi i \boldsymbol{\eta} \cdot\left(\boldsymbol{y}-\boldsymbol{y}^{\prime}\right)}}{\int_{\mathbb{R}^{n}} e^{4 \pi[\boldsymbol{\eta} \cdot \boldsymbol{v}-\tau \tilde{b}(\boldsymbol{v})]} d \boldsymbol{v}} d \boldsymbol{\eta} .
$$

Then by Corollary 5.13 and Claim 5.9 it follows that 


$$
\begin{aligned}
|S| \leq & \sum_{j=0}^{N+1} c_{j}\left|\int_{0}^{\frac{\pi}{|u|}} e^{-i u \tau} F\left(\tau+\frac{j \pi}{|u|}\right) d \tau\right| \\
& +\frac{1}{2^{N+1}}\left|\int_{\frac{\pi}{|u|}}^{\infty} e^{-i u \tau} \int_{0}^{\frac{\pi}{|u|}} \cdots \int_{0}^{\frac{\pi}{|u|}} F^{(N+1)}\left(\tau+s_{1}+\ldots+s_{N+1}\right) d s_{1} \cdots d s_{N+1} d \tau\right| .
\end{aligned}
$$

We will begin by obtaining the desired bound for the terms of the form

$$
I_{j}(u)=\int_{0}^{\frac{\pi}{|u|}} e^{-i u \tau} F\left(\tau+\frac{j \pi}{|u|}\right) d \tau
$$

We can write

$$
\left|I_{j}\right| \leq \int_{0}^{\frac{\pi}{|u|}}\left|F\left(\tau+\frac{j \pi}{|u|}\right)\right| d \tau=\int_{0}^{\frac{\pi}{|u|}}\left|e^{-2 \pi \delta\left(\tau+\frac{j \pi}{|u|}\right)} \int_{\mathbb{R}^{n}} \frac{e^{2 \pi i \boldsymbol{\eta} \cdot\left(\boldsymbol{y}-\boldsymbol{y}^{\prime}\right)}}{\int_{\mathbb{R}^{n}} e^{4 \pi\left[\boldsymbol{\eta} \cdot \boldsymbol{v}-\left(\tau+\frac{j \pi}{|u|}\right) \tilde{b}(\boldsymbol{v})\right]} d \boldsymbol{v}} d \boldsymbol{\eta}\right| d \tau .
$$

Making the change of variables $s=\tau+\frac{j \pi}{|u|}$ as well as $\boldsymbol{\eta} \rightarrow \frac{\boldsymbol{\eta}}{\boldsymbol{\mu}}$ and $\boldsymbol{v} \rightarrow \boldsymbol{\mu} \boldsymbol{v}$, we have that

$$
\left|I_{j}(u)\right| \leq \int_{\frac{j \pi}{|u|}}^{\frac{(j+1) \pi}{|u|}} \frac{e^{-2 \pi \delta s}}{\mu_{1}^{2} \cdots \mu_{n}^{2}} \int_{\mathbb{R}^{n}} \frac{1}{\int_{\mathbb{R}^{n}} e^{4 \pi[\boldsymbol{\eta} \cdot \boldsymbol{v}-s \tilde{b}(\boldsymbol{\mu} \boldsymbol{v})]} d \boldsymbol{v}} d \boldsymbol{\eta} d s
$$

By Lemma 4.1,

$$
\int_{\mathbb{R}^{n}} \frac{1}{\int_{\mathbb{R}^{n}} e^{4 \pi[\boldsymbol{\eta} \cdot \boldsymbol{v}-s \tilde{b}(\boldsymbol{\mu} \boldsymbol{v})]} d \boldsymbol{v}} d \boldsymbol{\eta}
$$

converges. Also, by convexity of $b, \delta>0$. Thus,

$$
\left|I_{j}(u)\right| \lesssim \int_{\frac{j \pi}{|u|}}^{\frac{(j+1) \pi}{|u|}} \frac{1}{\mu_{1}^{2} \cdots \mu_{n}^{2}} d s .
$$

By the choice of the factors $\boldsymbol{\mu}$, it follows that

$$
\left|I_{j}(u)\right| \lesssim \int_{\frac{j \pi}{|u|}}^{\frac{(j+1) \pi}{|u|}} \frac{1}{\left|\left\{\boldsymbol{v}: \widetilde{b}(\boldsymbol{v}) \leq \frac{1}{\tau}\right\}\right|^{2}} d \tau
$$

But since we are considering $\tau \leq \frac{(j+1) \pi}{|u|}$, then

$$
\left|I_{j}(u)\right| \lesssim \int_{\frac{j \pi}{|u|}}^{\frac{(j+1) \pi}{|u|}} \frac{1}{\left|\left\{\boldsymbol{v}: \widetilde{b}(\boldsymbol{v}) \leq \frac{|u|}{\pi(j+1)}\right\}\right|^{2}} d \tau=\frac{\pi}{|u|\left|\left\{\boldsymbol{v}: \widetilde{b}(\boldsymbol{v}) \leq \frac{|u|}{\pi(j+1)}\right\}\right|^{2}}
$$

Moreover, since $u=2 \pi w$, the desired bound follows immediately for $j=0$ and $j=1$. For $j>1$, it follows from Claim 6.1 (see Appendix) that

$$
|\{\boldsymbol{v}: \widetilde{b}(\boldsymbol{v}) \leq|w|\}| \leq \frac{(j+1)^{n}}{2^{n}}\left|\left\{\boldsymbol{v}: \widetilde{b}(\boldsymbol{v}) \leq \frac{2|w|}{(j+1)}\right\}\right| .
$$


Hence,

Since the sum over $j$ is finite, it follows that

$$
\left|I_{j}(w)\right| \lesssim \frac{(j+1)^{2 n}}{|w||\{\boldsymbol{v}: \widetilde{b}(\boldsymbol{v}) \leq|w|\}|^{2}}
$$

$$
\begin{aligned}
& |S| \lesssim \frac{1}{|w||\{\boldsymbol{v}: \widetilde{b}(\boldsymbol{v}) \leq|w|\}|^{2}} \\
& +\frac{1}{2^{N+1}}\left|\int_{\frac{\pi}{|u|}}^{\infty} e^{-i u \tau} \int_{0}^{\frac{\pi}{|u|}} \cdots \int_{0}^{\frac{\pi}{|u|}} F^{(N+1)}\left(\tau+s_{1}+\ldots+s_{N+1}\right) d s_{1} \cdots d s_{N+1} d \tau\right| .
\end{aligned}
$$

We must now bound the term

$$
\left|\int_{\frac{\pi}{|u|}}^{\infty} e^{-i u \tau} \int_{0}^{\frac{\pi}{|u|}} \cdots \int_{0}^{\frac{\pi}{|u|}} F^{(N+1)}\left(\tau+s_{1}+\ldots+s_{N+1}\right) d s_{1} \cdots d s_{N+1} d \tau\right| .
$$

As previously discussed, the $(N+1)^{t h}$ derivative of $F(\tau)$ consists of sums of terms of the form

$$
\frac{C(\tau \delta)^{N+1-k} e^{-2 \pi \tau \delta}}{\tau^{N+1}} \int_{\mathbb{R}^{n}} \frac{e^{2 \pi i \boldsymbol{\eta} \cdot\left(\boldsymbol{y}-\boldsymbol{y}^{\prime}\right)} f_{1}(\tau, \boldsymbol{\eta}) \cdots f_{k}(\tau, \boldsymbol{\eta})}{\gamma(\tau, \boldsymbol{\eta})} d \boldsymbol{\eta},
$$

where $f_{s}(\tau, \boldsymbol{\eta})=\left[\int_{\mathbb{R}^{n}}(\tau \widetilde{b}(\boldsymbol{v}))^{s} e^{4 \pi[\boldsymbol{\eta} \cdot \boldsymbol{v}-\tau \tilde{b}(\boldsymbol{v})]} d \boldsymbol{v}\right]^{a_{s}} ; \gamma(\tau, \boldsymbol{\eta})=\left[\int_{\mathbb{R}^{n}} e^{4 \pi[\boldsymbol{\eta} \cdot \boldsymbol{v}-\tau \tilde{b}(\boldsymbol{v})]} d \boldsymbol{v}\right]^{d} ; a_{1}, \ldots, a_{k}, k, d \in$ $\mathbb{N} ; 0 \leq k \leq N+1 ; a_{1}+\ldots+a_{k}=d-1 ;$ and $a_{1}+2 a_{2}+\ldots+k a_{k}=k$. These terms can be written as $C \tau^{-(N+1)} \Delta_{N+1, k}(\tau)$, where

$$
\Delta_{N+1, k}(\tau)=(\tau \delta)^{N+1-k} e^{-2 \pi \tau \delta} \int_{\mathbb{R}^{n}} \frac{e^{2 \pi i \boldsymbol{\eta} \cdot\left(\boldsymbol{y}-\boldsymbol{y}^{\prime}\right)} f_{1}(\tau, \boldsymbol{\eta}) \cdots f_{k}(\tau, \boldsymbol{\eta})}{\gamma(\tau, \boldsymbol{\eta})} d \boldsymbol{\eta}
$$

Thus, we must study integrals of the form

$$
J=\int_{\frac{\pi}{|u|}}^{\infty} \frac{e^{-i u \tau}}{\tau^{N+1}} \int_{0}^{\frac{\pi}{|u|}} \cdots \int_{0}^{\frac{\pi}{|u|}} \Delta_{N+1, k}\left(\tau+s_{1}+\ldots+s_{N+1}\right) d s_{1} \cdots d s_{N+1} d \tau .
$$

With $\mu_{1}, \ldots, \mu_{n}$ chosen as in equation (47) on page 27 we can make the changes of variable $\boldsymbol{\eta} \rightarrow \frac{\eta}{\mu}$ and $\boldsymbol{v} \rightarrow \boldsymbol{\mu} \boldsymbol{v}$. We obtain an integral of the form

$$
J=\int_{\frac{\pi}{|u|}}^{\infty} \frac{\left(\mu_{1} \cdots \mu_{n}\right)^{a_{1}+\ldots+a_{k}}}{\tau^{N+1}\left(\mu_{1} \cdots \mu_{n}\right)^{d+1}} \int_{0}^{\frac{\pi}{|u|}} \cdots \int_{0}^{\frac{\pi}{|u|}} \Delta_{N+1, k}^{\mu}\left(\tau+s_{1}+\ldots+s_{N+1}\right) d s_{1} \cdots d s_{N+1} d \tau
$$

where now

$$
\Delta_{N+1, k}^{\mu}(\tau)=(\tau \delta)^{N+1-k} e^{-2 \pi \tau \delta} \int_{\mathbb{R}^{n}} \frac{e^{2 \pi i \frac{\boldsymbol{\eta}}{\boldsymbol{\mu}} \cdot\left(\boldsymbol{y}-\boldsymbol{y}^{\prime}\right)} f_{1}^{\mu}(\tau, \boldsymbol{\eta}) \cdots f_{k}^{\mu}(\tau, \boldsymbol{\eta})}{\gamma^{\mu}(\tau, \boldsymbol{\eta})} d \boldsymbol{\eta}
$$

is as in Claim 5.11. Thus, $\left|\Delta_{N+1, k}^{\mu}(\tau)\right| \leq C$. It follows that 
Also, $a_{1}+\ldots+a_{k}=d-1$. Thus,

$$
|J| \lesssim \int_{\frac{\pi}{|u|}}^{\infty} \frac{\left(\mu_{1} \cdots \mu_{n}\right)^{a_{1}+\ldots+a_{k}}}{|u|^{N+1} \tau^{N+1}\left(\mu_{1} \cdots \mu_{n}\right)^{d+1}} d \tau
$$

$$
|J| \lesssim \frac{1}{|u|^{N+1}} \int_{\frac{\pi}{|u|}}^{\infty} \frac{1}{\tau^{N+1}\left(\mu_{1} \cdots \mu_{n}\right)^{2}} d \tau
$$

Since $u=2 \pi w$, and by choice of $\boldsymbol{\mu}$, it follows that

$$
|J| \lesssim \frac{1}{|w|^{N+1}} \int_{\frac{1}{2|w|}}^{\infty} \frac{1}{\tau^{N+1}\left|\left\{\boldsymbol{v}: \widetilde{b}(\boldsymbol{v}) \leq \frac{1}{\tau}\right\}\right|^{2}} d \tau .
$$

Notice that on the interval under consideration, $(2|w| \tau)^{-1} \leq 1$. Using Claim 6.1, it follows that

$$
\left|\left\{\boldsymbol{v}: \widetilde{b}(\boldsymbol{v}) \leq \frac{1}{\tau}\right\}\right|=\left|\left\{\boldsymbol{v}: \widetilde{b}(\boldsymbol{v}) \leq \frac{2|w|}{2|w| \tau}\right\}\right| \geq \frac{1}{(2|w| \tau)^{n}}|\{\boldsymbol{v}: \widetilde{b}(\boldsymbol{v}) \leq 2|w|\}| .
$$

Thus, and since $|\{\boldsymbol{v}: \widetilde{b}(\boldsymbol{v}) \leq 2|w|\}| \geq|\{\boldsymbol{v}: \widetilde{b}(\boldsymbol{v}) \leq|w|\}|$ It follows that

Taking $N \geq 2 n+1$, we have that

$$
\left|\left\{\boldsymbol{v}: \widetilde{b}(\boldsymbol{v}) \leq \frac{1}{\tau}\right\}\right| \geq \frac{1}{(2|w| \tau)^{n}}|\{\boldsymbol{v}: \widetilde{b}(\boldsymbol{v}) \leq|w|\}| .
$$

$$
\left.|J| \lesssim \frac{|w|^{2 n}}{|w|^{N+1}} \int_{\frac{1}{2|w|}}^{\infty} \frac{\tau^{2 n}}{\tau^{N+1}|\{\boldsymbol{v}: \widetilde{b}(\boldsymbol{v}) \leq|w|\}|^{2}} d \tau \approx \frac{1}{|w|^{N+1-2 n}|\{\boldsymbol{v}: \widetilde{b}(\boldsymbol{v}) \leq|w|\}|^{2}} \cdot \tau^{2 n-N}\right|_{\frac{1}{2|w|}} ^{\infty} .
$$

That is,

It follows from equation (71) that

$$
|J| \lesssim \frac{1}{|w||\{\boldsymbol{v}: \tilde{b}(\boldsymbol{v}) \leq|w|\}|^{2}}
$$

This finishes the proof of our third and last bound.

$$
|S| \lesssim \frac{1}{|w||\{\boldsymbol{v}: \tilde{b}(\boldsymbol{v}) \leq|w|\}|^{2}}
$$

5.2.4. Conclusion. We have shown that

$$
\left|S\left((\boldsymbol{x}, \boldsymbol{y}, t) ;\left(\boldsymbol{x}^{\prime}, \boldsymbol{y}^{\prime}, t^{\prime}\right)\right)\right| \lesssim \min \{A, B, C\},
$$

where

$$
\begin{gathered}
A=\frac{1}{\delta|\{\boldsymbol{v}: \tilde{b}(\boldsymbol{v})<\delta\}|^{2}} \\
B=\frac{1}{\widetilde{b}\left(\boldsymbol{y}-\boldsymbol{y}^{\prime}\right)\left|\left\{\boldsymbol{v}: \tilde{b}(\boldsymbol{v})<\widetilde{b}\left(\boldsymbol{y}-\boldsymbol{y}^{\prime}\right)\right\}\right|^{2}}
\end{gathered}
$$


and

$$
C=\frac{1}{|w||\{\boldsymbol{v}: \widetilde{b}(\boldsymbol{v})<|w|\}|^{2}} .
$$

Thus, to conclude the proof of the Main Theorem, it suffices to show that

$$
\min \{A, B, C\} \lesssim \frac{1}{\sqrt{\delta^{2}+\widetilde{b}\left(\boldsymbol{y}-\boldsymbol{y}^{\prime}\right)^{2}+w^{2}}\left|\left\{\boldsymbol{v}: \tilde{b}(\boldsymbol{v})<\sqrt{\delta^{2}+\widetilde{b}\left(\boldsymbol{y}-\boldsymbol{y}^{\prime}\right)^{2}+w^{2}}\right\}\right|^{2}}
$$

Without loss of generality, suppose that $\delta \leq \widetilde{b}\left(\boldsymbol{y}-\boldsymbol{y}^{\prime}\right) \leq|w|$. Then,

$$
\frac{1}{|w|} \leq \frac{\sqrt{3}}{\sqrt{\delta^{2}+\widetilde{b}\left(\boldsymbol{y}-\boldsymbol{y}^{\prime}\right)^{2}+w^{2}}}
$$

and

But by Claim 6.1,

$$
\left\{\boldsymbol{v}: \tilde{b}(\boldsymbol{v})<\sqrt{\delta^{2}+\widetilde{b}\left(\boldsymbol{y}-\boldsymbol{y}^{\prime}\right)^{2}+w^{2}}\right\} \subseteq\{\boldsymbol{v}: \tilde{b}(\boldsymbol{v})<\sqrt{3}|w|\} .
$$

Therefore,

$$
|\{\boldsymbol{v}: \tilde{b}(\boldsymbol{v})<\sqrt{3}|w|\}|^{2} \leq(\sqrt{3})^{2 n}|\{\boldsymbol{v}: \tilde{b}(\boldsymbol{v})<|w|\}|^{2}
$$

$$
\begin{aligned}
|S| & \lesssim \frac{1}{|w||\{\boldsymbol{v}: \widetilde{b}(\boldsymbol{v})<|w|\}|^{2}} \\
& \leq \frac{(\sqrt{3})^{2 n+1}}{\sqrt{\delta^{2}+\widetilde{b}\left(\boldsymbol{y}-\boldsymbol{y}^{\prime}\right)^{2}+w^{2}}\left|\left\{\boldsymbol{v}: \tilde{b}(\boldsymbol{v})<\sqrt{\delta^{2}+\widetilde{b}\left(\boldsymbol{y}-\boldsymbol{y}^{\prime}\right)^{2}+w^{2}}\right\}\right|^{2}}
\end{aligned}
$$

This finishes the proof of the Main Theorem.

\section{Appendix}

We have included in this appendix two technical claims for convex functions that are used repeatedly throughout the paper.

Claim 6.1. Let $f: \mathbb{R}^{n} \rightarrow \mathbb{R}$ be a convex function such that $f(\mathbf{0})=0$. Given $x>0$, let

$$
A_{x}=\left\{\boldsymbol{w} \in \mathbb{R}^{n}: f(\boldsymbol{w}) \leq x\right\}
$$

Then for any constant $0 \leq \lambda \leq 1, \operatorname{vol}\left(A_{\lambda x}\right) \geq \lambda^{n} \operatorname{vol}\left(A_{x}\right)$.

Proof. By convexity of $f$, for any vectors $\boldsymbol{w}$ and $\boldsymbol{u}$ in $\mathbb{R}^{n}$, and any constant $0 \leq \lambda \leq 1$,

$$
f(\lambda \boldsymbol{w}+(1-\lambda) \boldsymbol{u}) \leq \lambda f(\boldsymbol{w})+(1-\lambda) f(\boldsymbol{u}) .
$$

In particular, taking $\boldsymbol{u}=\mathbf{0}$, and since by hypothesis $f(\mathbf{0})=0$, it follows that $f(\lambda \boldsymbol{w}) \leq \lambda f(\boldsymbol{w})$. Thus, if $\boldsymbol{w} \in A_{x}$, then $f(\lambda \boldsymbol{w}) \leq \lambda f(\boldsymbol{w}) \leq \lambda x$. That is, $\lambda \cdot A_{x} \subseteq A_{\lambda x}$. It follows that

$$
\operatorname{vol}\left(A_{\lambda x}\right) \geq \operatorname{vol}\left(\lambda \cdot A_{x}\right)=\lambda^{n} \operatorname{vol}\left(A_{x}\right)
$$


Claim 6.2. If $f: \mathbb{R}^{n} \rightarrow \mathbb{R}$ is a convex function such that $f(\mathbf{0})=0$ and $\nabla f(\mathbf{0})=0$ then

$$
I=\int_{\mathbb{R}^{n}} e^{-f(\boldsymbol{w})} d \boldsymbol{w} \approx|\{\boldsymbol{w}: f(\boldsymbol{w}) \leq 1\}| .
$$

Proof. Without loss of generality we can assume $f \not \equiv 0$. Notice that under these hypothesis $f(\boldsymbol{v}) \geq$ $0 \forall \boldsymbol{v} \in \mathbb{R}^{n}$. A lower bound for $I$ can be easily obtained, since

$$
\int_{\mathbb{R}^{n}} e^{-f(\boldsymbol{w})} d \boldsymbol{w} \geq \int_{\{\boldsymbol{w}: f(\boldsymbol{w}) \leq 1\}} e^{-f(\boldsymbol{w})} d \boldsymbol{w} \geq \frac{1}{e}|\{\boldsymbol{w}: f(\boldsymbol{w}) \leq 1\}| .
$$

To obtain an upper bound we can write

$$
\int_{\mathbb{R}^{n}} e^{-f(\boldsymbol{w})} d \boldsymbol{w}=\int_{\{\boldsymbol{w}: f(\boldsymbol{w}) \leq 1\}} e^{-f(\boldsymbol{w})} d \boldsymbol{w}+\sum_{j=1}^{\infty} \int_{\{\boldsymbol{w}: j \leq f(\boldsymbol{w}) \leq j+1\}} e^{-f(\boldsymbol{w})} d \boldsymbol{w}
$$

But

$$
\int_{\{\boldsymbol{w}: j \leq f(\boldsymbol{w}) \leq j+1\}} e^{-f(\boldsymbol{w})} d \boldsymbol{w} \leq e^{-j}|\{\boldsymbol{w}: f(\boldsymbol{w}) \leq j+1\}|
$$

But by Claim 6.1 , and taking $\lambda=\frac{1}{j+1}$ and $x=j+1$, it follows that

$$
|\{\boldsymbol{w}: f(\boldsymbol{w}) \leq j+1\}| \leq(j+1)^{n}|\{\boldsymbol{w}: f(\boldsymbol{w}) \leq 1\}| .
$$

Hence, by equation (74), we have that

$$
I \leq|\{\boldsymbol{w}: f(\boldsymbol{w}) \leq 1\}|\left(1+\sum_{j=1}^{\infty} e^{-j}(j+1)^{n}\right) .
$$

Since the sum converges we get the desired upper bound.

\section{ACKnowledgments}

The results presented in this paper first appeared in my Ph.D. thesis [1]. I would like to thank my advisor Alexander Nagel for his guidance and contributions to this work. I would also like to thank an anonymous referee for many insightful comments and suggestions. This work was partially supported by a doctoral fellowship from CONICYT (Chile).

\section{REFERENCES}

[1] S. Benguria, Estimates for the Szego kernel on unbounded convex domains, PhD thesis, University of WisconsinMadison, 2014.

[2] H. P. BoAs, Regularity of the Szegő projection in weakly pseudoconvex domains, Indiana Univ. Math. J., 34 (1985), pp. 217-223.

[3] — The Szegö projection: Sobolev estimates in regular domains, Trans. Amer. Math. Soc., 300 (1987), pp. 109132.

[4] L. Boutet de Monvel and J. Sjöstrand, Sur la singularité des noyaux de Bergman et de Szegö, in Journées: Équations aux Dérivées Partielles de Rennes (1975), Soc. Math. France, Paris, 1976, pp. 123-164. Astérisque, No. $34-35$. 
[5] J. Bruna, A. Nagel, And S. Wainger, Convex hypersurfaces and Fourier transforms, Ann. of Math. (2), 127 (1988), pp. 333-365.

[6] C. Carracino, Estimates for the Szegö kernel on a model non-pseudoconvex domain, Illinois J. Math., 51 (2007), pp. $1363-1396$.

[7] M. ChRist, Regularity properties of the $\bar{\partial}_{b}$ equation on weakly pseudoconvex CR manifolds of dimension 3 , J. Amer. Math. Soc., 1 (1988), pp. 587-646.

[8] C. Fefferman, The Bergman kernel and biholomorphic mappings of pseudoconvex domains, Invent. Math., 26 (1974), pp. 1-65.

[9] C. L. Fefferman, J. J. Kohn, And M. Machedon, Hölder estimates on CR manifolds with a diagonalizable Levi form, Adv. Math., 84 (1990), pp. 1-90.

[10] G. Francsics And N. Hanges, Explicit formulas for the Szegö kernel on certain weakly pseudoconvex domains, Proc. Amer. Math. Soc., 123 (1995), pp. 3161-3168.

[11] M. Gilliam And J. Halfpap, The Szegő kernel for certain non-pseudoconvex domains in $\mathbb{C}^{2}$, Illinois J. Math., 55 (2011), pp. 871-894.

[12] - The Szegö kernel for nonpseudoconvex tube domains in $\mathbb{C}^{2}$, Complex Var. Elliptic Equ., 59 (2014), pp. 769786.

[13] S. G. Gindikin, Analysis in homogeneous domains, Uspehi Mat. Nauk, 19 (1964), pp. 3-92.

[14] P. Greiner And E. Stein, On the solvability of some differential operators of type $\square_{b}$, Proc. Internat. Conf. (Cortona, Italy, 1976-1977), (1978), pp. 106-165.

[15] J. Halfpap, A. Nagel, And S. Wainger, The Bergman and Szegő kernels near points of infinite type, Pacific J. Math., 246 (2010), pp. 75-128.

[16] F. Haslinger, Singularities of the Szegő kernel for certain weakly pseudoconvex domains in $\mathbf{C}^{2}$, J. Funct. Anal., 129 (1995), pp. 406-427.

[17] F. John, Extremum problems with inequalities as subsidiary conditions, in Studies and Essays Presented to R. Courant on his 60th Birthday, January 8, 1948, Interscience Publishers, Inc., New York, N. Y., 1948, pp. 187-204.

[18] S. G. KRAntz, Holomorphic functions of bounded mean oscillation and mapping properties of the Szegö projection, Duke Math. J., 47 (1980), pp. 743-761.

[19] — A direct connection between the Bergman and Szegö projections, Complex Anal. Oper. Theory, 8 (2014), pp. $571-579$.

[20] L. Lanzani And E. M. Stein, Szegö and Bergman projections on non-smooth planar domains, J. Geom. Anal., 14 (2004), pp. 63-86.

[21] — Cauchy-type integrals in several complex variables, Bull. Math. Sci., 3 (2013), pp. 241-285.

[22] M. Machedon, Szegö kernels on pseudoconvex domains with one degenerate eigenvalue, Ann. of Math. (2), 128 (1988), pp. 619-640.

[23] J. D. McNeal, Estimates on the Bergman kernels of convex domains, Adv. Math., 109 (1994), pp. $108-139$.

[24] J. D. McNeal and E. M. Stein, The Szegö projection on convex domains, Math. Z., 224 (1997), pp. $519-553$.

[25] A. NAGEL, Vector fields and nonisotropic metrics, in Beijing lectures in harmonic analysis (Beijing, 1984), vol. 112 of Ann. of Math. Stud., Princeton Univ. Press, Princeton, NJ, 1986, pp. 241-306.

[26] A. Nagel, J.-P. Rosay, E. M. Stein, And S. Wainger, Estimates for the Bergman and Szegő kernels in certain weakly pseudoconvex domains, Bull. Amer. Math. Soc. (N.S.), 18 (1988), pp. 55-59.

[27] A. Nagel, J.-P. Rosay, E. M. Stein, And S. Wainger, Estimates for the Bergman and Szegö kernels in $\mathbf{C}^{2}$, Ann. of Math. (2), 129 (1989), pp. 113-149.

[28] A. Peterson, Estimates for the Szego Projection on Uniformly Finite-Type Subdomains of $\mathbb{C}^{2}$, ArXiv e-prints, (2015).

[29] D. H. Phong And E. M. Stein, Estimates for the Bergman and Szegö projections on strongly pseudo-convex domains, Duke Math. J., 44 (1977), pp. 695-704.

[30] A. RAICh AND M. TinkeR, The Szegö kernel on a class of non-compact CR manifolds of high codimension, Complex Var. Elliptic Equ., 60 (2015), pp. 1366-1373.

[31] N. Wiener, The Fourier Integral and Certain of its Applications, Dover Publications, Inc., New York, 1959.

Mathematics Department, University of Wisconsin - Madison, 480 Lincoln Dr, Madison, Wi, USA

E-mail address: benguria@math.wisc.edu 